\title{
Amphiphilic Hyperbranched Polyethoxysiloxane: A Self-Templating Assembled Platform to Fabricate Functionalized Mesostructured Silicas for Aqueous Enantioselective Reactions
}

Kun Zhang, Juzeng An, Yanchao Su, Jueyu Zhang, Ziyun Wang, Tanyu Cheng, Guohua $\mathrm{Liu}^{*}$

Key Laboratory of Resource Chemistry of Ministry of Education, Shanghai Key Laboratory of Rare Earth

Functional Materials, Shanghai Normal University, Shanghai, China. Tel: + 8621 64322280; E-mail: ghliu@shnu.edu.cn.

\begin{tabular}{|c|c|c|}
\hline & Content & Page \\
\hline Experimental & General, preparation and catalysis & S2 \\
\hline Figure S1 & FT-IR spectra of catalyst 3, and the SH-MSNs and catalyst 5 & S5 \\
\hline Figure S2 & $\begin{array}{l}\text { XPS spectra of the homogeneous MesityleneRuArDPEN and } \\
\text { catalyst } 3\end{array}$ & S6 \\
\hline Figure S3 & $\begin{array}{l}\text { Solid-state }{ }^{29} \mathrm{Si} \mathrm{CP} \text { MAS NMR spectra of catalyst } \mathbf{3} \text {, the } \mathrm{SH} \text { - } \\
\text { MSNs and catalyst } 5 \text {. }\end{array}$ & S7 \\
\hline Figure S4 & $\begin{array}{l}\text { Nitrogen adsorption-desorption isotherms of catalyst } \mathbf{3} \text {, the } \mathrm{SH} \text { - } \\
\text { MSNs and catalyst } \mathbf{5} \text {. }\end{array}$ & S8 \\
\hline Figure S5 & $\begin{array}{l}\text { (a) The TG/DTA curves of the ArDPEN/PEOS and catalyst } \mathbf{3} \text {, } \\
\text { and explanations. (b) The TG/DTA curves of the SH-MSNs and } \\
\text { catalyst } 5 \text {, and explanations. }\end{array}$ & s9 \\
\hline Figure S6 & $\begin{array}{l}\text { (a) SEM images of } \mathbf{3}^{\prime},(\mathrm{b}) \text { TEM images of } \mathbf{3}^{\prime}, \text { (c) SEM images of } \\
\mathbf{5} \text {, (d) TEM images of } \mathbf{5} \text {, and (e) the dispersive situation of } \\
\text { catalyst } \mathbf{5} \text { in water. }\end{array}$ & S12 \\
\hline Fig ure S7 & $\begin{array}{l}\text { Solid-state }{ }^{13} \mathrm{C} \text { CP MAS NMR spectra of the SH-MSNs and } \\
\text { catalyst } 5 .\end{array}$ & S14 \\
\hline Figure S8 & Asymmetric transfer hydrogenation of $\alpha$-trifluoromethylimines & S15 \\
\hline Table S1 & $\begin{array}{l}\text { Reusability of catalyst } 3 \text { using 4-methoxy-N-(2,2,2-trifluoro-1-(4- } \\
\text { fluorophenyl)ethylidene)aniline as a substrate }\end{array}$ & S30 \\
\hline Figure S9 & $\begin{array}{l}\text { Reusability of catalyst } 3 \text { using 4-methoxy-N-(2,2,2-trifluoro-1-(4- } \\
\text { fluorophenyl)ethylidene)aniline as a substrate }\end{array}$ & $\mathrm{S} 30$ \\
\hline Figure S10 & Asymmetric Michael addition of malonates to nitroalkenes & S33 \\
\hline Table S2 & $\begin{array}{l}\text { Reusability of catalyst } \mathbf{5} \text { for asymmetric Michael addition of } \\
\text { acetylacetone to nitrostyrene }\end{array}$ & S45 \\
\hline Figure S11 & $\begin{array}{l}\text { Reusability of catalyst } \mathbf{5} \text { for asymmetric Michael addition of } \\
\text { acetylacetone to nitrostyrene }\end{array}$ & S45 \\
\hline
\end{tabular}




\section{Experimental}

\section{General}

All experiments, which are sensitive to moisture or air, were carried out under an $\mathrm{Ar}$ atmosphere using the standard Schlenk techniques. (S,S)-1,2-diphenylenediamine, $\left[\mathrm{RuCl}_{2}\right.$ (mesitylene)]2, 3-mercaptopropyltrimethoxylsilane, acetic anhydride (98\%), tetraethyl orthotitanate (95\%), poly(ethylene glycol) monomethyl ether (average molecular weight 350), tetraethoxysilane (TEOS) were purchased from Sigma-Aldrich Company Ltd. Compounds poly(ethyleneglycol) monomethylether-modified hyperbranched polyethoxysiloxane (PEGPEOS), 3-((3,5-bis(trifluoromethyl)benzyl)amino)-4-(((1S)-(6-methoxyquinolin-4-yl)((2S)-5vinylquinuclidin-2-yl)methyl)amino)cyclobut-3-ene-1,2-dione and (trimethoxysilyl)ethyl)phenylsulfonyl-1,2-diphenylethylenediamine was synthesized according to the reported methods.

\section{General procedure for the reuse experiments using 4-methoxy-N-(2,2,2-trifluoro-1- (4-fluorophenyl)ethylidene)aniline as a substrate.}

The catalyst 3 (195.60 mg, $40.0 \mu \mathrm{mol}$ of Ru based on ICP analysis), 4-methoxy-N-(2,2,2trifluoro-1-(4-fluorophenyl)ethylidene)aniline $(2.0 \mathrm{mmol})$, and $10.0 \mathrm{mmol}$ of HCOONa in 20.0 $\mathrm{mL}$ of water were added sequentially to a $50.0 \mathrm{~mL}$ round-bottom flask. The mixture was then stirred at room temperature $\left(40^{\circ} \mathrm{C}\right)$ for $16 \mathrm{~h}$. After completion of the reaction, the catalyst was separated by centrifugation $(10,000 \mathrm{rpm})$. The collected solids was transfered to a fresh $50.0 \mathrm{~mL}$ round-bottom flask and 4-methoxy-N-(2,2,2-trifluoro-1-(4fluorophenyl)ethylidene)aniline $(2.0 \mathrm{mmol})$, and $10.0 \mathrm{mmol}$ of $\mathrm{HCOONa}$ in $20.0 \mathrm{~mL}$ of water were added sequentially again for next recycle. The aqueous solution was extracted with ethyl ether $(3 \times 3.0 \mathrm{~mL})$. The combined ethyl ether extracts were washed with $\mathrm{NaHCO}_{3}$ and brine, and then dehydrated with $\mathrm{Na}_{2} \mathrm{SO}_{4}$. After evaporation of ethyl ether, the residue was purified by silica gel flash column chromatography to afford the desired product, determining its yield and ee value.

3. Preparation of the inorganosilicate analogue (3'). In a typical procedure, $1.0 \mathrm{~g}(0.36$ mmol) of PEOS (Macromol. Chem. Phys. 2003, 204, 1014) was dispersed in $20.0 \mathrm{~mL}$ of deionized water and was treated by ultrasonic irradiation for $10 \mathrm{~min}$. Then an ammonia aqueous solution (25\%) $(1.20 \mathrm{~mL}$ ) was added to adjust the ammonia concentration to $0.7 \mathrm{M}$. The mixture was kept under gentle stirring $(500 \mathrm{rpm})$ at $25^{\circ} \mathrm{C}$ for $3 \mathrm{~h}$. After that, $0.10 \mathrm{~g}(0.20$ mmol) of 2 was added to the solution. The reaction mixture was stirred at $25^{\circ} \mathrm{C}$ with a stirring speed of $500 \mathrm{rpm}$ for another $24 \mathrm{~h}$. The solids were collected by centrifugation and 
washed repeatedly with excess distilled water. The solid was filtered, rinsed with ethanol again, and then dried at $60{ }^{\circ} \mathrm{C}$ under reduced pressure overnight to afford ArDPENfunctionalized PEOS $(0.71 \mathrm{~g})$ in the form of a white powder. The collected solids $(0.50 \mathrm{~g})$ were suspended in $20.0 \mathrm{~mL}$ of dry $\mathrm{CH}_{2} \mathrm{Cl}_{2}$ again and $0.10 \mathrm{~g}$ of $(0.17 \mathrm{mmol})$ $\left[\mathrm{RuCl}_{2} \text { (mesitylene) }\right]_{2}$ were added. The resulting mixture was stirred at $25{ }^{\circ} \mathrm{C}$ for $24 \mathrm{~h}$. The mixture was filtered through filter paper and then rinsed with excess $\mathrm{CH}_{2} \mathrm{Cl}_{2}$. After Soxhlet extraction for $10 \mathrm{~h}$ in $\mathrm{CH}_{2} \mathrm{Cl}_{2}$ to remove homogeneous and unreacted starting materials, the solid was dried at ambient temperature under vacuum overnight to afford catalyst 3' $(0.47 \mathrm{~g})$ as a light-yellow powder.

4. Preparation of catalyst (5). In a typical procedure, $1.0 \mathrm{~g}(0.36 \mathrm{mmol})$ of PEG-PEOS was dispersed in $16.0 \mathrm{~mL}$ of deionized water and was treated by ultrasonic irradiation for $10 \mathrm{~min}$. Then an ammonia aqueous solution (25\%) (18.0 mL) was added to adjust the ammonia concentration to $0.7 \mathrm{M}$. The mixture was kept under gentle stirring (500 rpm) at $25^{\circ} \mathrm{C}$ for $3 \mathrm{~h}$. After that, $0.10 \mathrm{~g}(0.42 \mathrm{mmol})$ of (triethoxysilyl)propanethiol was added to the solution. The reaction mixture was stirred at $25^{\circ} \mathrm{C}$ with a stirring speed of $500 \mathrm{rpm}$ for another $24 \mathrm{~h}$. The solids were collected by centrifugation and washed repeatedly with excess distilled water. The solid was filtered, rinsed with ethanol again, and then dried at $60{ }^{\circ} \mathrm{C}$ under reduced pressure overnight to afford SH-MSNs $(0.73 \mathrm{~g})$ in the form of a white powder. Then a dry 50 $\mathrm{mL}$ round-bottom flask was charged with the collected SH-MSNs solids $(0.50 \mathrm{~g})$, squaramide (4) (65.0 mg, $0.10 \mathrm{mmol})$, and $2.0 \mathrm{~mol} \%$ of 2,2-dimethoxy-1,2diphenylethanone photoinitiator, backfilled with argon, and irradiated for $27 \mathrm{~h}$ with a $15 \mathrm{~W}$ blacklight $\left(\lambda_{\max }=365 \mathrm{~nm}\right)$. The resulting solid was filtered through filter paper and rinsed with excess methanol and dichloromethane. After Soxlet extraction in dichloromethane solvent for $12 \mathrm{~h}$ to remove any homogeneous and unreacted starting materials, the solid was dried overnight at $60^{\circ} \mathrm{C}$ under vacuum to afford heterogeneous catalyst (5) $(0.48 \mathrm{~g})$ as a light-yellow powder. IR (KBr) cm $\mathrm{cm}^{-1}$ : 3417.7 (s), 2977.6 (w), $2930.2(\mathrm{w}), 2921.1$ (w), 1666.2 $(\mathrm{w}), 1630.1$ (m), 1591.7 (w), 1535.3 (w), 1451.8 (w), 1386.3 (w), 1086.1 (s), $964.3(\mathrm{~m})$,

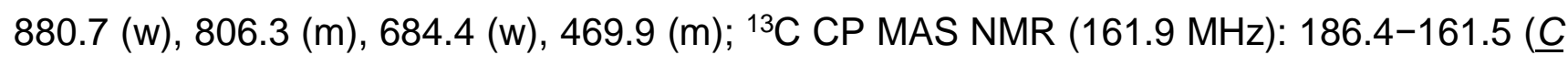
of $\underline{C}=\mathrm{O}$ and $-\mathrm{CO} \underline{C} \mathrm{H}=\underline{C} \mathrm{HCO}^{-}$), 160.1-114.0 ( $\underline{C}$ of $\mathrm{Ar}, \mathrm{Ph}$ and $\left.\mathrm{CF}_{3}\right), 70.1 \quad\left(\underline{\mathrm{C}} \mathrm{H}_{2}\right.$ of $-\mathrm{O}_{\underline{C}} \mathrm{H}_{2} \underline{\mathrm{C}} \mathrm{H}_{2} \mathrm{O}-$ ), 56.1 ( $\underline{\mathrm{C}}$ of $-\mathrm{O} \underline{\mathrm{C}} \mathrm{H}_{3}$ in aromatic ring), 48.2-34.9 ( $\underline{C}$ of carbon connected to $\mathrm{N}$ atom in alkyl part, and $\underline{C}$ of $-\underline{S}_{\underline{C}} H_{2}$, and $-{ }^{-} \underline{C} H_{3}$ in PEG molecule), 32.8-18.9 ( $\underline{C}$ of $-\mathrm{SiCH}_{2} \underline{\mathrm{C}} \mathrm{H}_{2} \mathrm{CH}_{2} \mathrm{~N}, \underline{\boldsymbol{C}}$ of cyclic alkyl groups in cycling group without connected to $\mathrm{N}$ atom), 15.9-5.8 ( $\underline{C}$ of $-\mathrm{Si} \underline{\underline{C}} \mathrm{H}_{2}$ group) ppm; ${ }^{29} \mathrm{Si} \mathrm{MAS}$ NMR $(79.4 \mathrm{MHz}): \mathrm{T}^{2}(\delta=-57.7 \mathrm{ppm}), \mathrm{T}^{3}(\delta=$ $-68.0 \mathrm{ppm}), \mathrm{Q}^{2}(\delta=-93.0 \mathrm{ppm}), \mathrm{Q}^{3}(\delta=-102.9 \mathrm{ppm}), \mathrm{Q}^{4}(\delta=-111.8 \mathrm{ppm})$. 
5. General procedure for asymmetric Michael addition of acetylacetone to nitroalkenes. A typical procedure was as follows: catalyst 5 (36.26 mg, $5.0 \mu \mathrm{mol}$ of squaramide based on TG analysis), nitroalkenes $(1.0 \mathrm{mmol})$, acetylacetone $(2.0 \mathrm{mmol})$ and $5.0 \mathrm{~mL}$ brine were added in a $10 \mathrm{~mL}$ round-bottom flask in turn. The mixture was allowed to react at $25^{\circ} \mathrm{C}$ for 20 minute. During that time, the reaction was monitored constantly by TLC. After completion of the reaction, the heterogeneous catalyst was filtered through filter paper for the recycle experiment. The aqueous solution was extracted by $\mathrm{Et}_{2} \mathrm{O}(3 \times 3.0 \mathrm{~mL})$. The combined $\mathrm{Et}_{2} \mathrm{O}$ was washed with brine twice and dehydrated with $\mathrm{Na}_{2} \mathrm{SO}_{4}$. After the evaporation of $\mathrm{Et}_{2} \mathrm{O}$, the residue was purified by silica gel flash column chromatography to afford the desired product. The conversion was calculated by the internal standard method and the ee value could be determined by chiral HPLC analysis with a UV-Vis detector using a Daicel chiralcel column $(\Phi 0.46 \times 25 \mathrm{~cm})$. The absolute configurations of compounds were assigned by comparison of their optical rotations to literature values. 

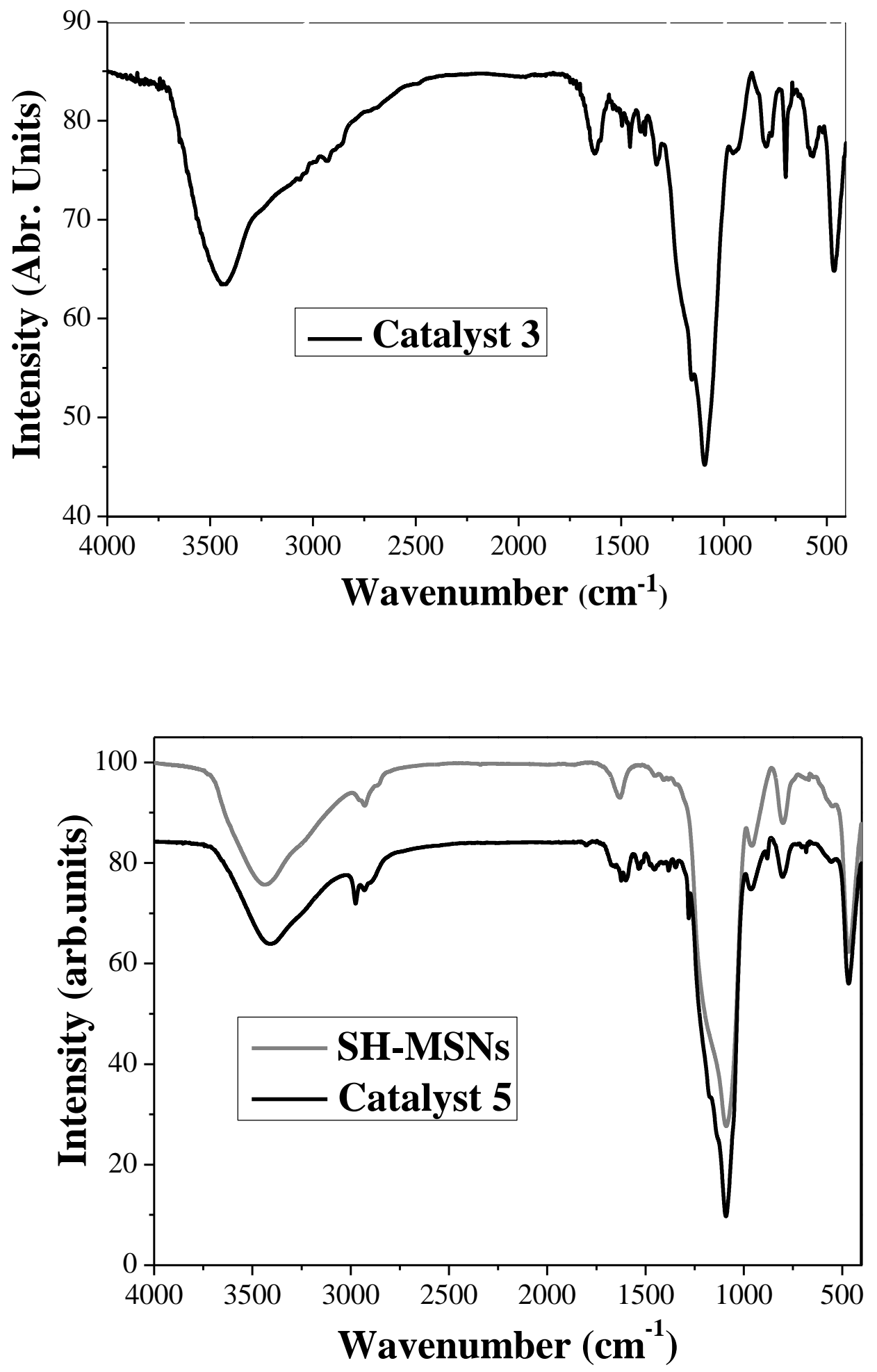

Figure S1. FT-IR spectra of catalyst 3, and the SH-MSNs and catalyst 5. 


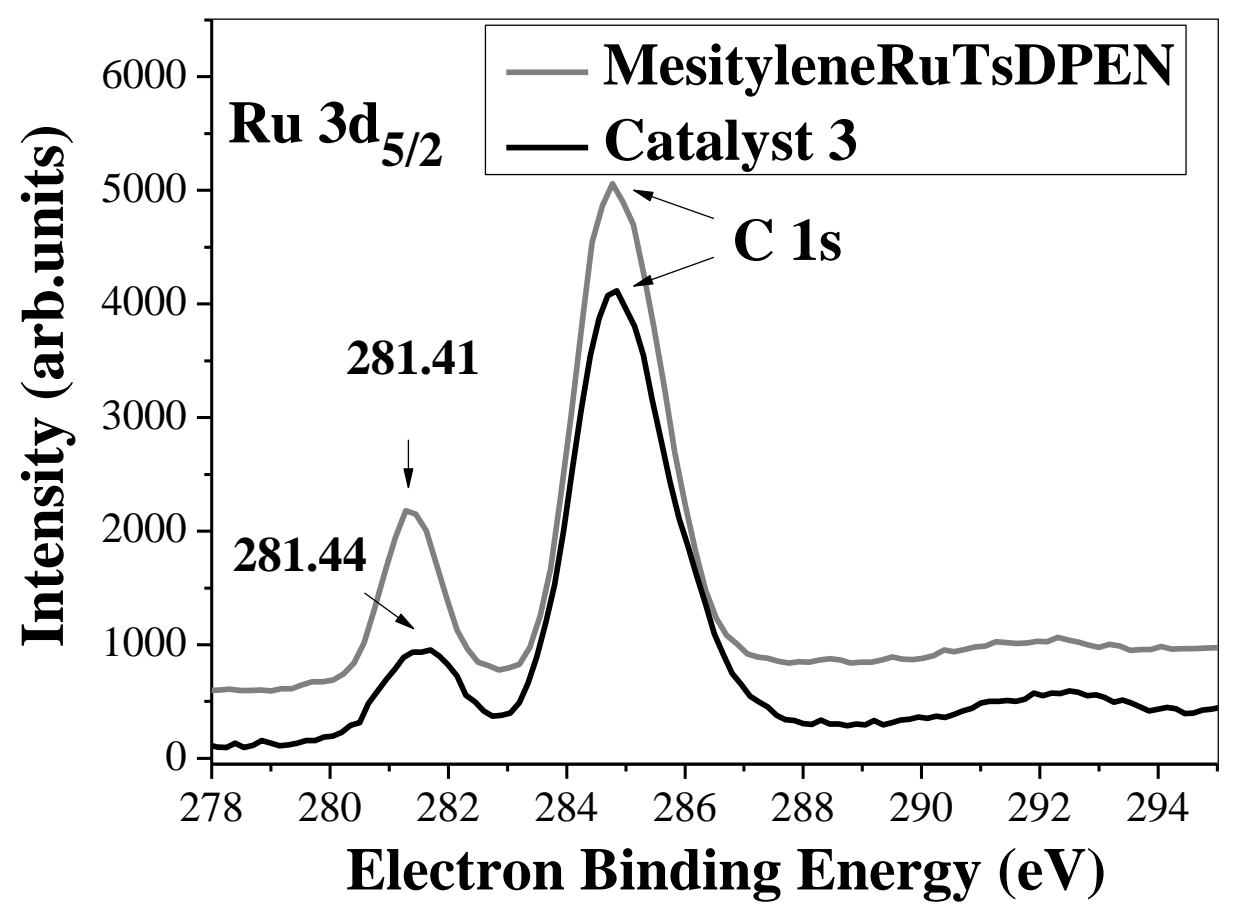

Figure S2. XPS spectra of the homogeneous MesityleneRuArDPEN and catalyst 3. 

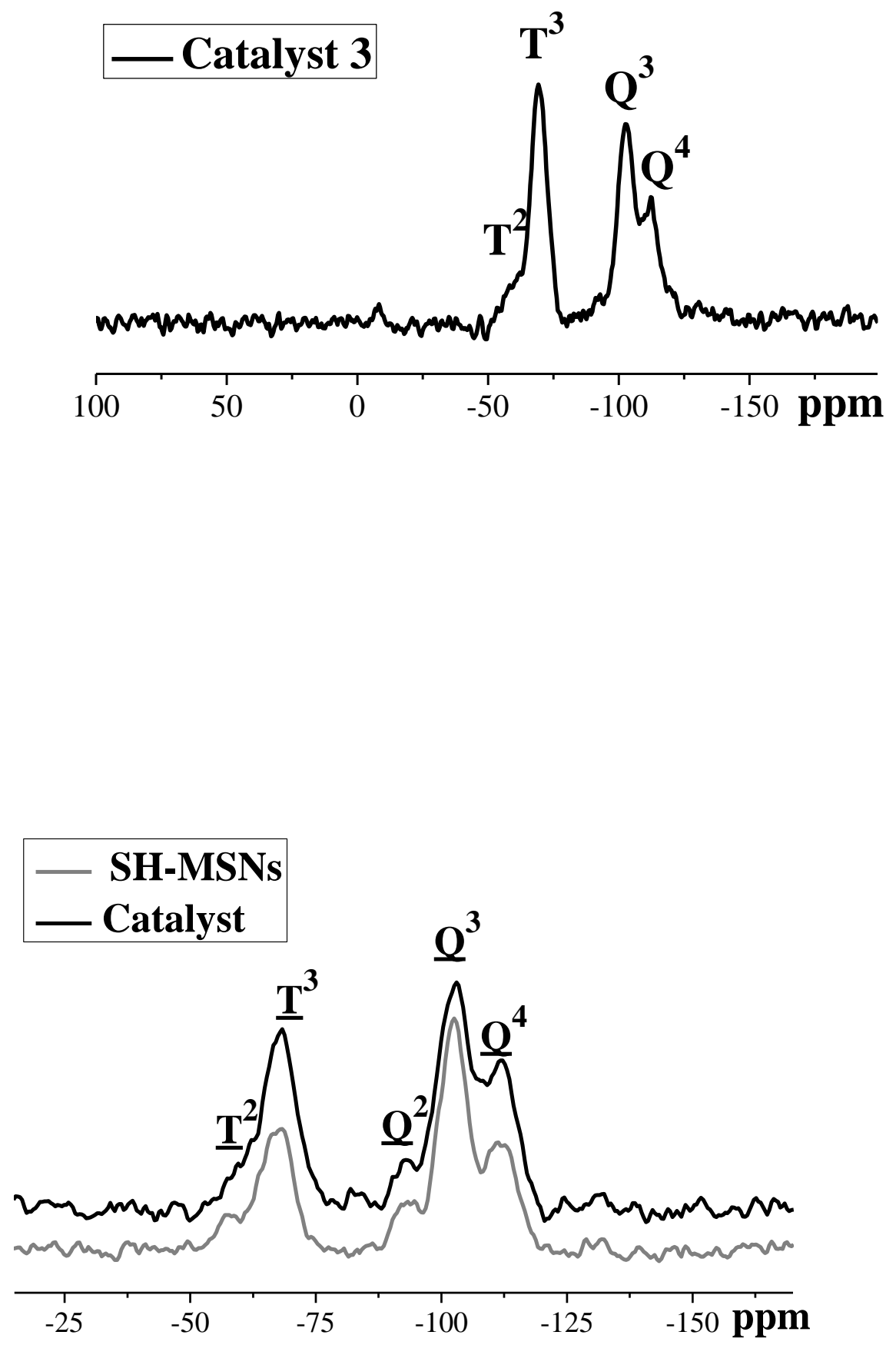

Figure S3. Solid-state ${ }^{29} \mathrm{Si} \mathrm{CP}$ MAS NMR spectra of catalyst 3, the SH-MSNs and catalyst 5. 

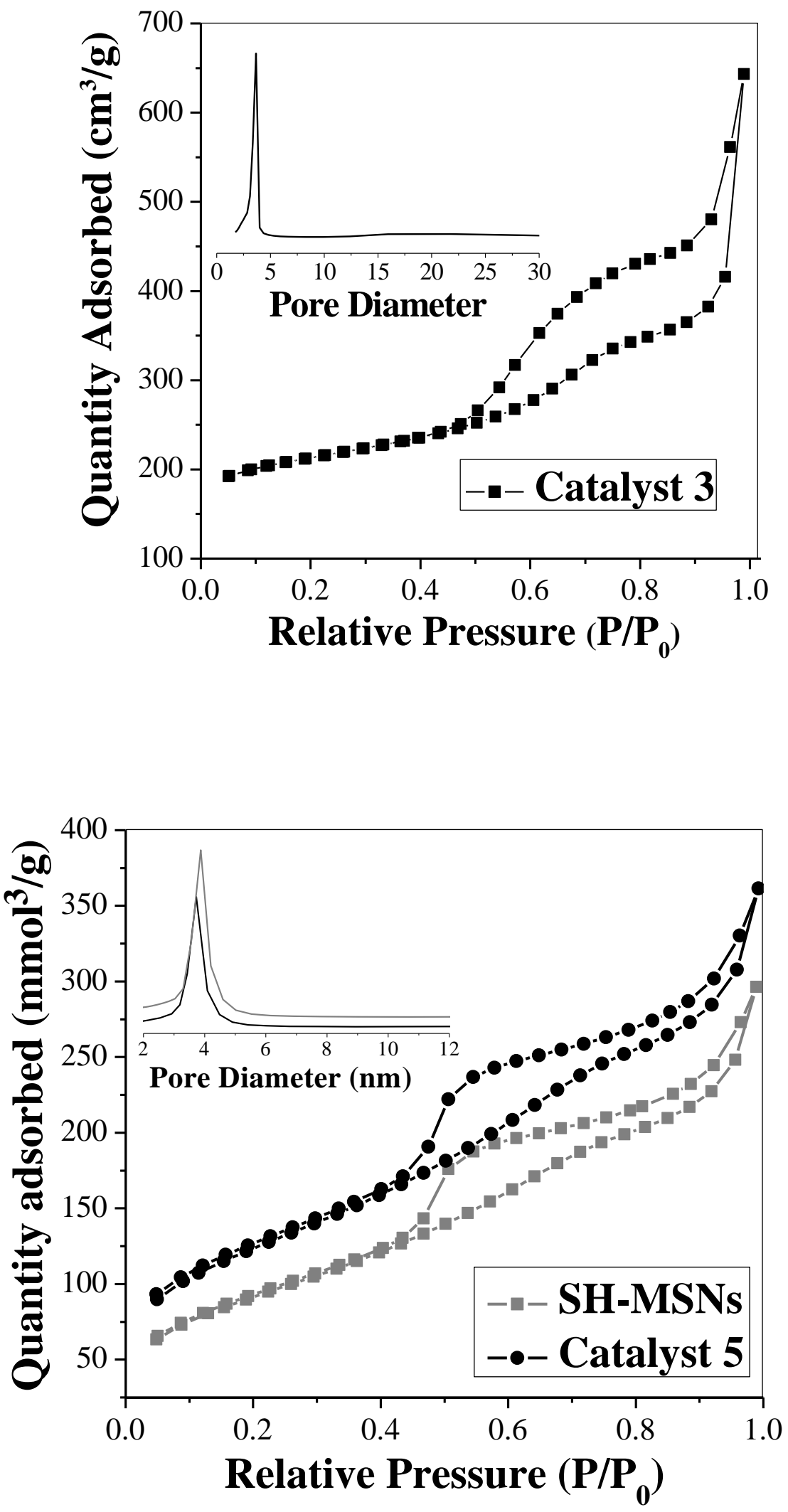

Figure S4. Nitrogen adsorption-desorption isotherms of catalyst $\mathbf{3}$, the SH-MSNs and catalyst 5 . 

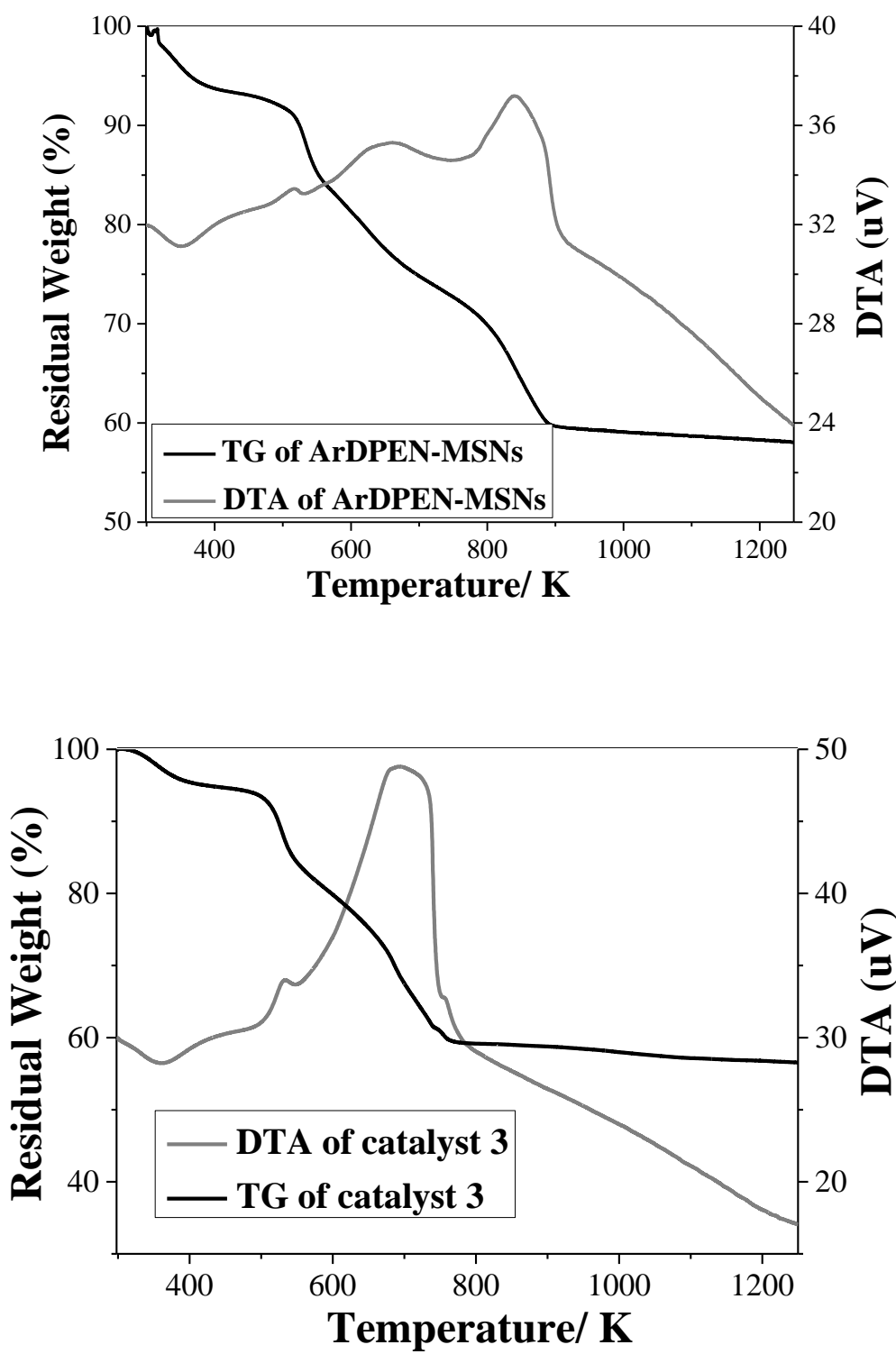

Figure S5. (a) The TG/DTA curves of the ArDPEN/PEOS and catalyst 3.

Explanations (Figure 5a): The TG/DTA curve of ArDPEN-functionalized mesostructured nanoparticles (ArDPEN-MSNs) was treated in the air as shown in above Figure. An endothermic peak around $350 \mathrm{~K}$ with weight loss of (100-92.82) $7.18 \%$ could be attributed to the release of physical adsorption water. In addition, the weight loss of (92.82-58.52) $34.30 \%$ between 460 and $1200 \mathrm{~K}$ could be assigned to the oxidation of the organic moieties (the oxidation of alkyl fragments, alkyl-linked PEG molecules and alkyl-linked ArDPEN groups in material). When eliminated the contribution of water, the total weight loss the organic moieties is $36.95 \%$.

For catalyst 3, an endothermic peak around $358 \mathrm{~K}$ with weight loss of (100-94.57) 5.43\% could be attributed to the release of physical adsorption water. In addition, the weight loss of 
(94.57-56.74) $37.83 \%$ between 460 and $1200 \mathrm{~K}$ could be assigned to the oxidation of the organic moieties (alkyl-linked diamine/ruthenium complex moieties, alkyl fragments, alkyllinked PEG molecules and alkyl-linked ArDPEN groups in material). When eliminated the contribution of water, the total weight loss the organic moieties is $40.00 \%$.

Thus, in contrast to TG/DTA curve of ArDPEN-MSNs and catalyst 3, the true weight loss of [MesityleneCl] organic moiety is 3.05 (40.00-36.95\%), meaning the mole amounts of [MesityleneCl] is $0.01968 \mathrm{mmol} \%(\mathrm{Mr}=155)$. The mole amount of [MesityleneCl] in the material is $0.1968 \mathrm{mmol}(20.07 \mathrm{mg} \mathrm{Ru}$ ) per gram material. This datum is nearly consistent with $20.65 \mathrm{mg}(0.204 \mathrm{mmol})$ of Ru loadings per gram catalyst detected by ICP analysis.
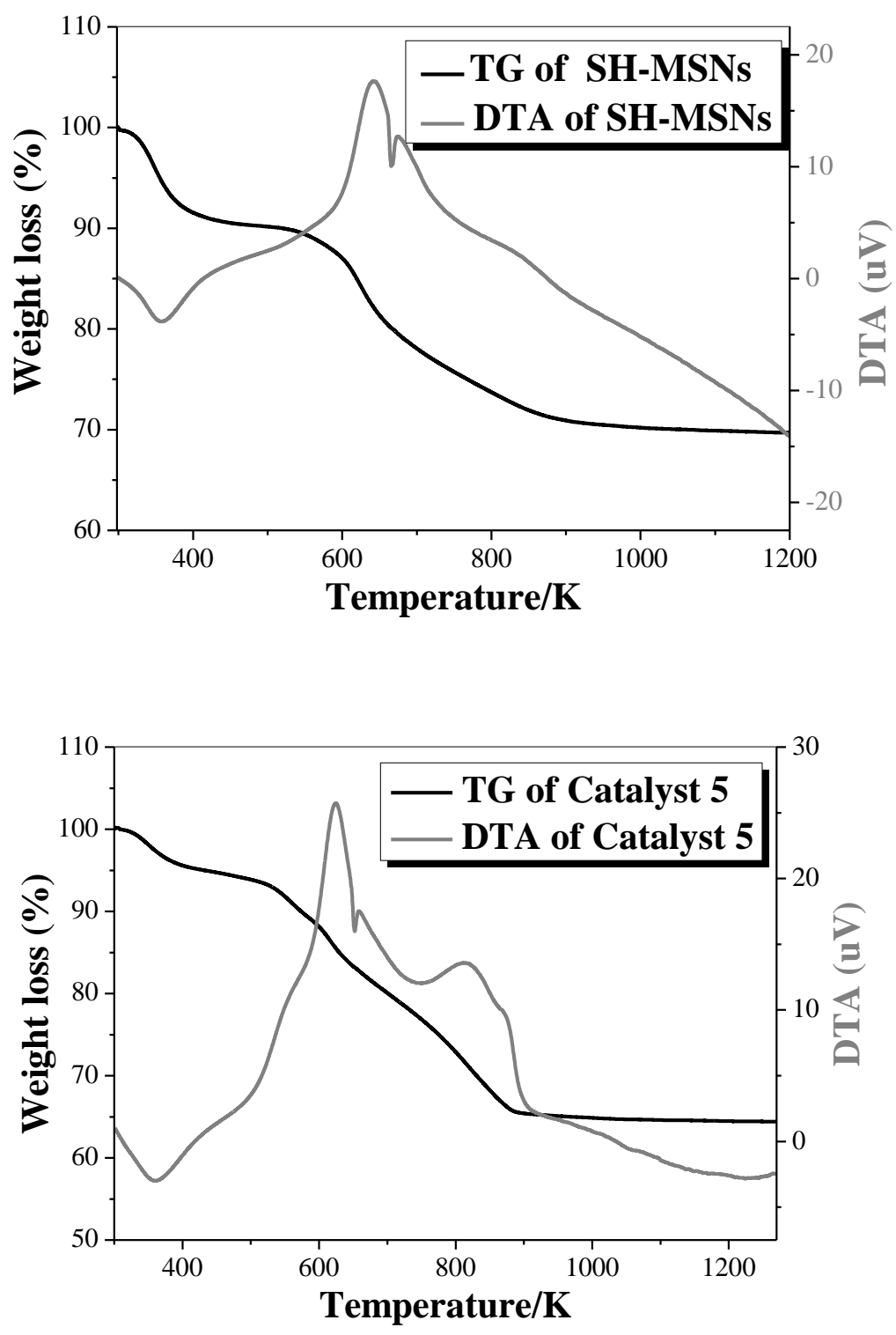

Figure S5. (b) The TG/DTA curves of the SH-MSNs and catalyst 5. 
Explanations (Figure 5b): The TG/DTA curve of the pure material (SH-MSNs) was treated in the air. An endothermic peak around $450 \mathrm{~K}$ with weight loss of (100-90.53) $8.47 \%$ could be attributed to the release of physical adsorption water. In addition, the weight loss of (90.53-69.68) $20.85 \%$ between 450 and $1200 \mathrm{~K}$ could be assigned to the organic moieties (the oxidation of alkyl fragments in material). When eliminated the contribution of water, the total weight loss the organic moieties is $23.03 \%$.

For catalyst 5, an endothermic peak around $450 \mathrm{~K}$ with weight loss of (100-94.73) 5.27\% could be attributed to the release of physical adsorption water. In addition, the weight loss of (94.73-64.47) $30.26 \%$ between 450 and $1200 \mathrm{~K}$ could be assigned to the oxidation of the organic moieties (alkyl fragments and propyl-linked squaramides in material). When eliminated the contribution of water, the total weight loss the organic moieties is $31.94 \%$.

Thus, in contrast to TG/DTA curve of the pure material (SH-MSNs) and catalyst 5 , the true weight loss of squaramide is $8.91 \%(31.94-23.03 \%)$, meaning the mole amounts of squaramide is $0.01379 \mathrm{mmol} \%(\mathrm{Mr}=646)$. The mole amount of squaramide in the material is $0.1379 \mathrm{mmol}(89.08 \mathrm{mg})$ per gram material. 

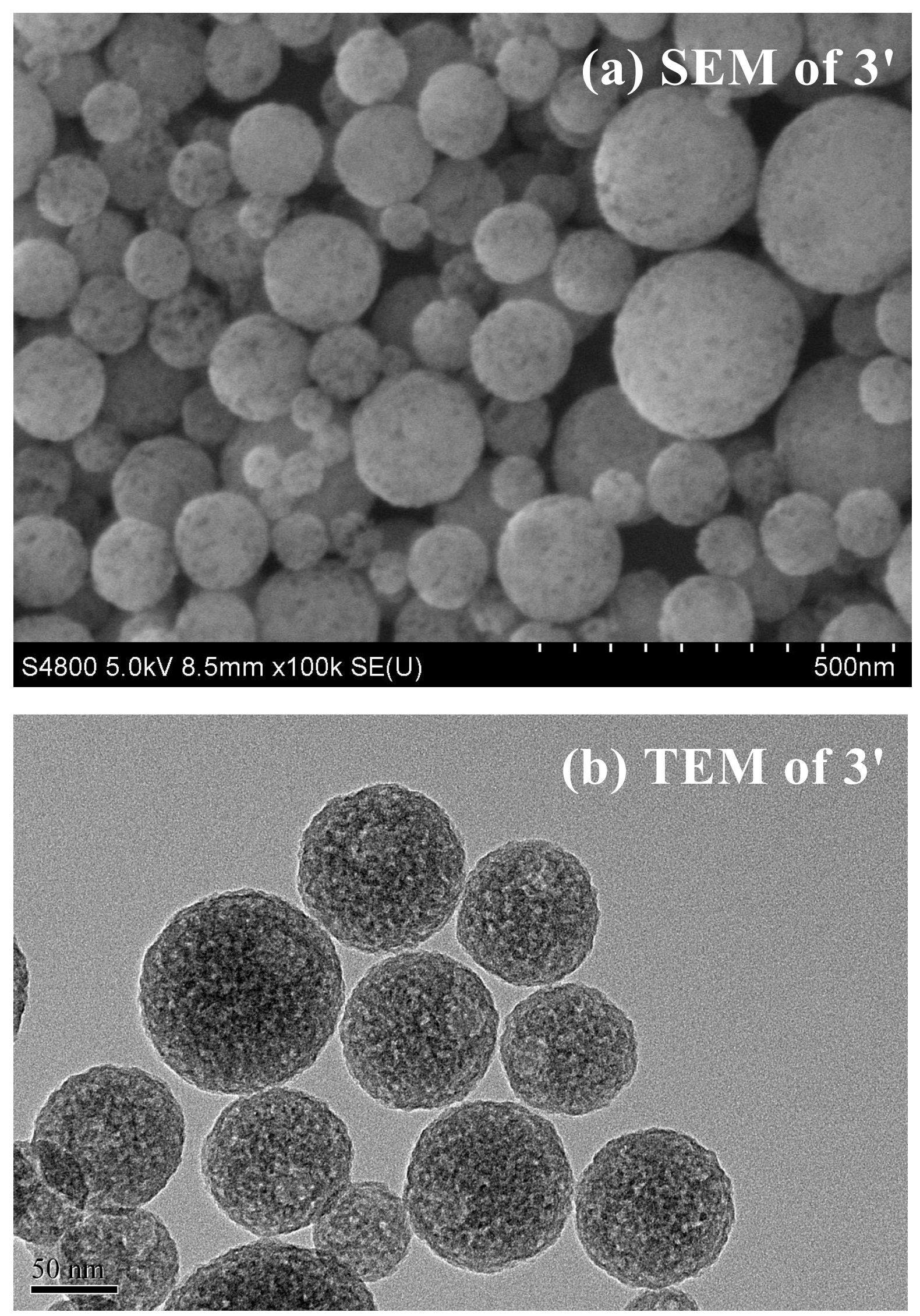

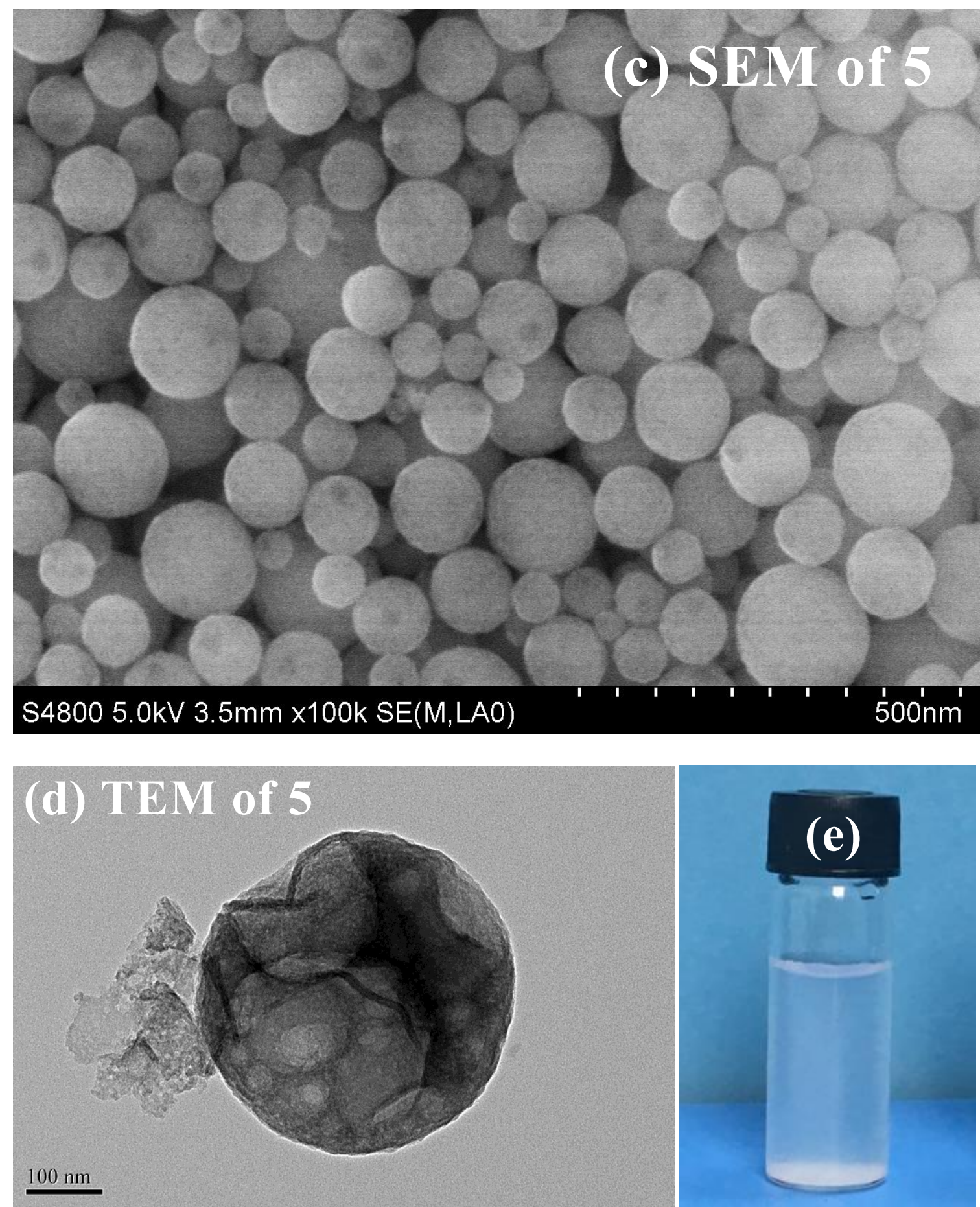

Figure 56. (a) SEM images of 3', (b) TEM images of 3', (c) SEM images of 5, (d) TEM images of 5 , and (e) the dispersive situation of catalyst 5 in water. 


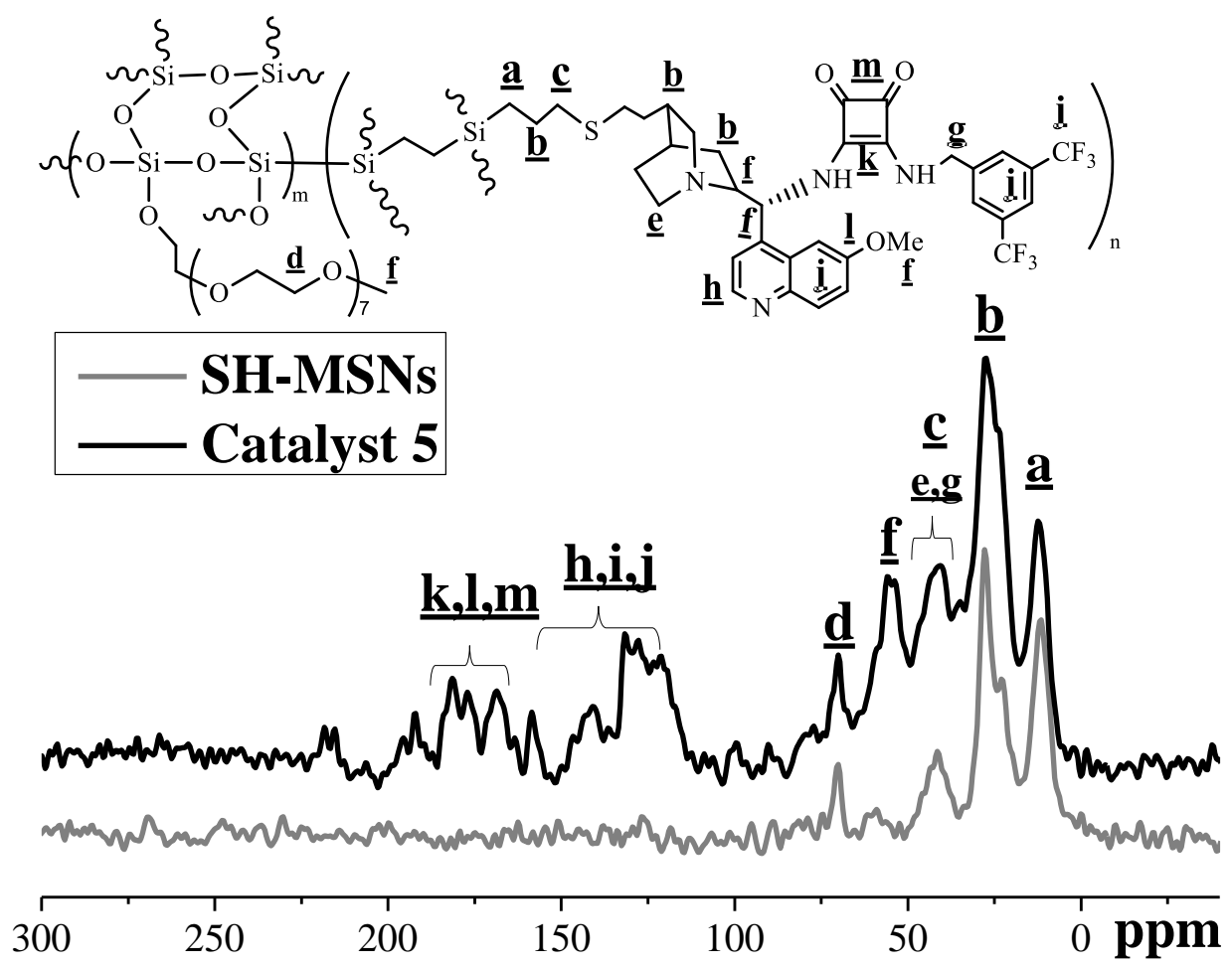

Figure S7. Solid-state ${ }^{13} \mathrm{C}$ CP MAS NMR spectra of the SH-MSNs and catalyst 5 
(S)-4-methoxy-N-(2,2,2-trifluoro-1-phenylethyl)aniline (HPLC: Chiracel OD-H, detected at $254 \mathrm{~nm}$, eluent: $\mathrm{n}$-hexane/2-propanol $=95 / 5$, flow rate $=1.0 \mathrm{~mL} / \mathrm{min}, 25^{\circ} \mathrm{C}, \mathrm{t}_{1}=8.9 \mathrm{~min}$ (maj), $\mathrm{t}_{2}=9.9 \mathrm{~min}$ )
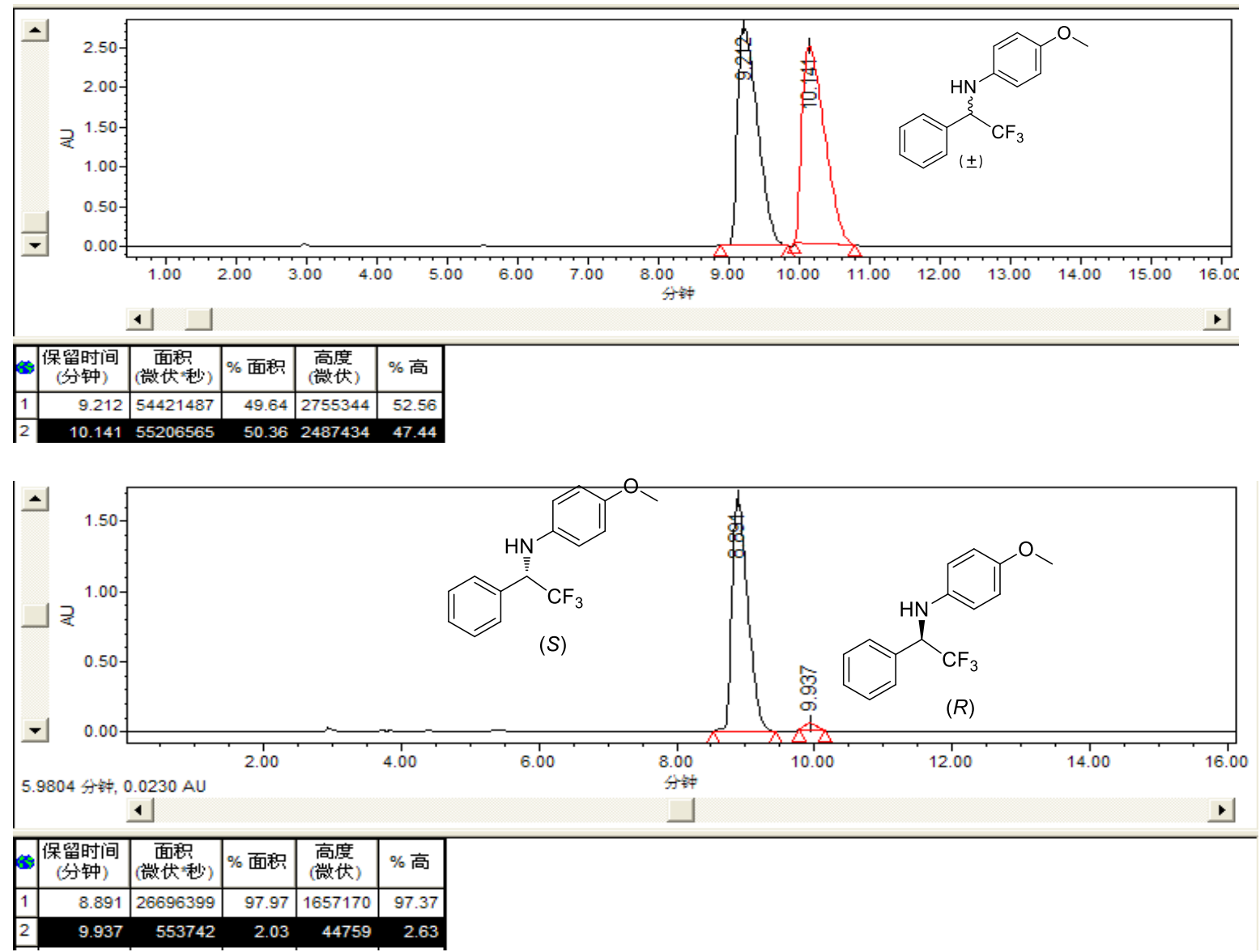

The hot-filtration experiment for ATH of 4-methoxy-N-(2,2,2-trifluoro-1-phenylethylidene)aniline for first $8 \mathrm{~h}$.

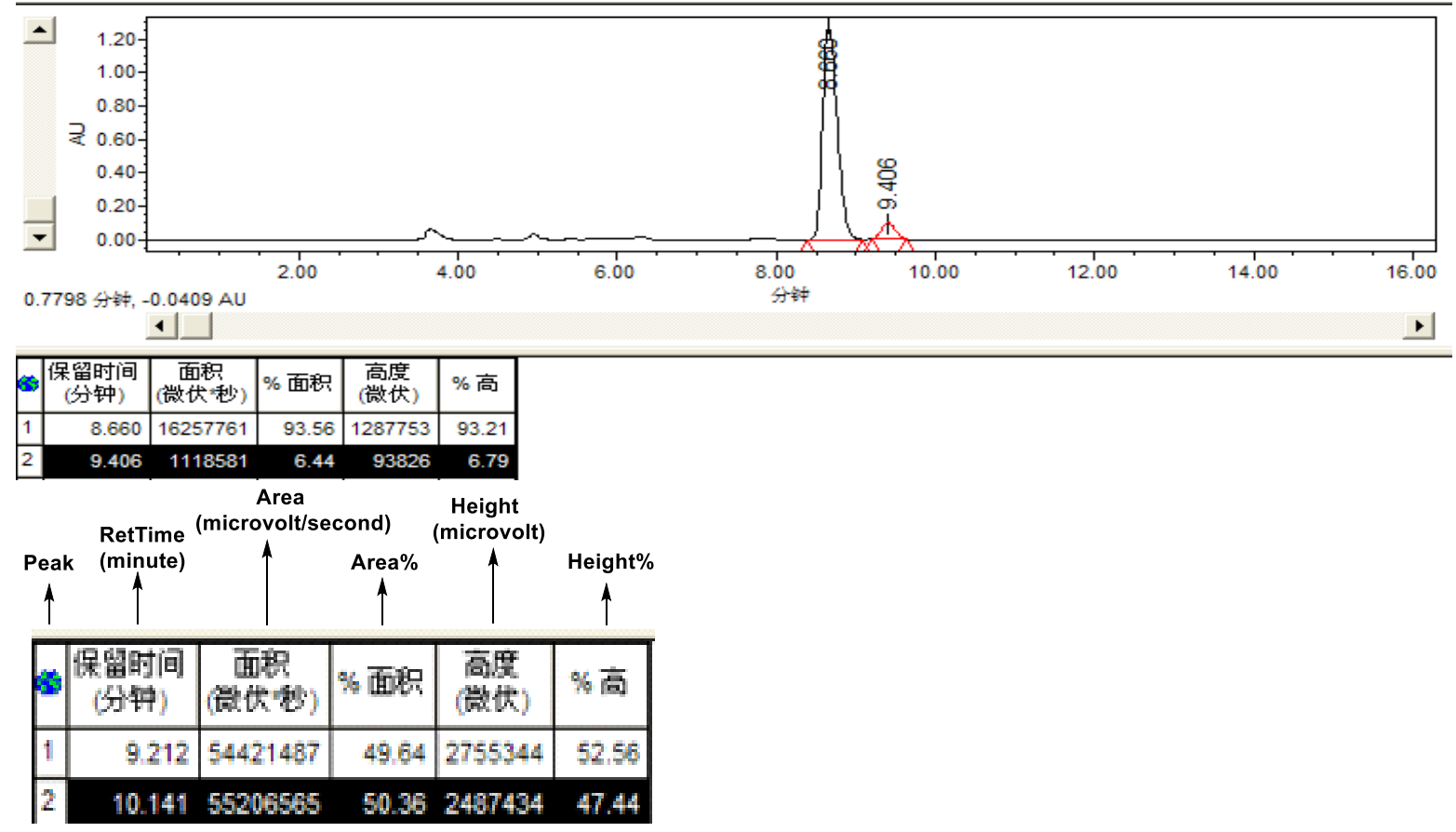


(S)-4-methoxy-N-(2,2,2-trifluoro-1-(4-fluorophenyl)ethyl)aniline: (HPLC: Chiracel OD-H, detector: $254 \mathrm{~nm}$, elute: Hexanes/i-PrOH $=95 / 5$, flow rate: $0.5 \mathrm{~mL} / \mathrm{min}, 25^{\circ} \mathrm{C}, \mathrm{t}_{1}=10.3 \mathrm{~min}$ (maj), t2 $=12.1 \mathrm{~min}$ ).

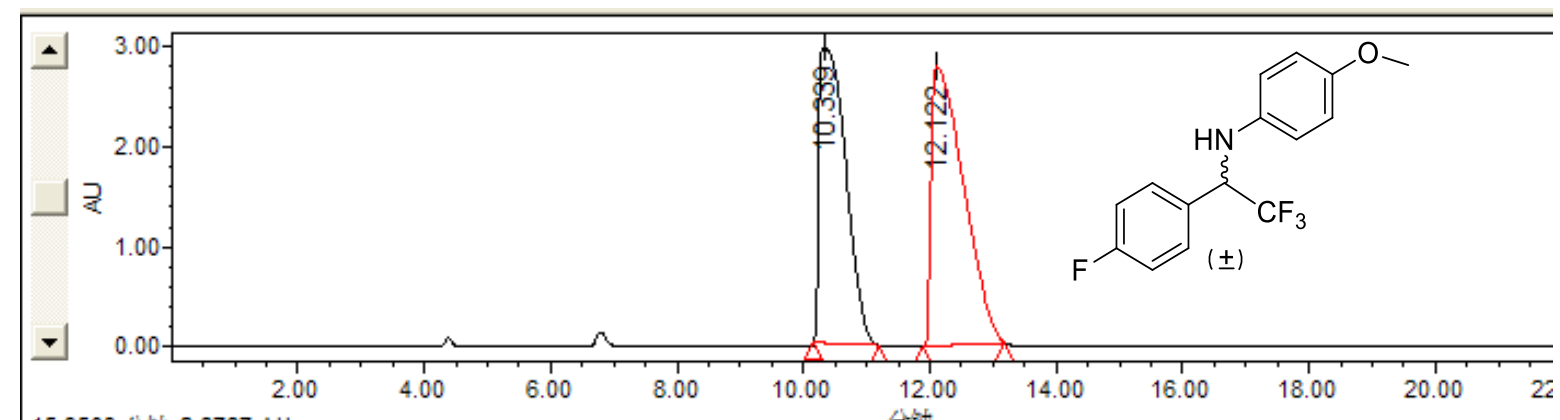

15.9503 分料, $2.6767 \mathrm{AU}$ 分神

\begin{tabular}{|c|c|c|c|c|c|c|c|c|c|c|c|c|c|c|c|}
\hline 6 & 名称 & $\begin{array}{c}\text { 保留时间 } \\
\text { (分钟) }\end{array}$ & $\begin{array}{c}\text { 面积 } \\
\text { (溦伏秒) }\end{array}$ & \% 面积 & $\begin{array}{l}\text { 高庶 } \\
\text { (溦伏) }\end{array}$ & 积分类型 & 含量 & 单位 & 㷨类型 & 窑代码 & \begin{tabular}{|c|} 
结构 1 \\
名
\end{tabular} & \begin{tabular}{|c|} 
结构 1 \\
说明
\end{tabular} & $\begin{array}{l}\text { 结构 } 1 \\
\text { 分子量 }\end{array}$ & \begin{tabular}{|l} 
结构 1 \\
公式
\end{tabular} & \begin{tabular}{|c|} 
结构 1 \\
结构
\end{tabular} \\
\hline 1 & & 10.339 & 86635345 & 46.79 & 2943658 & $\mathrm{bb}$ & & & 未知 & & & & & & (3) \\
\hline 2 & & 12.122 & 98527717 & 53.21 & 2789991 & bb & & & 未知 & & & & & & i \\
\hline
\end{tabular}

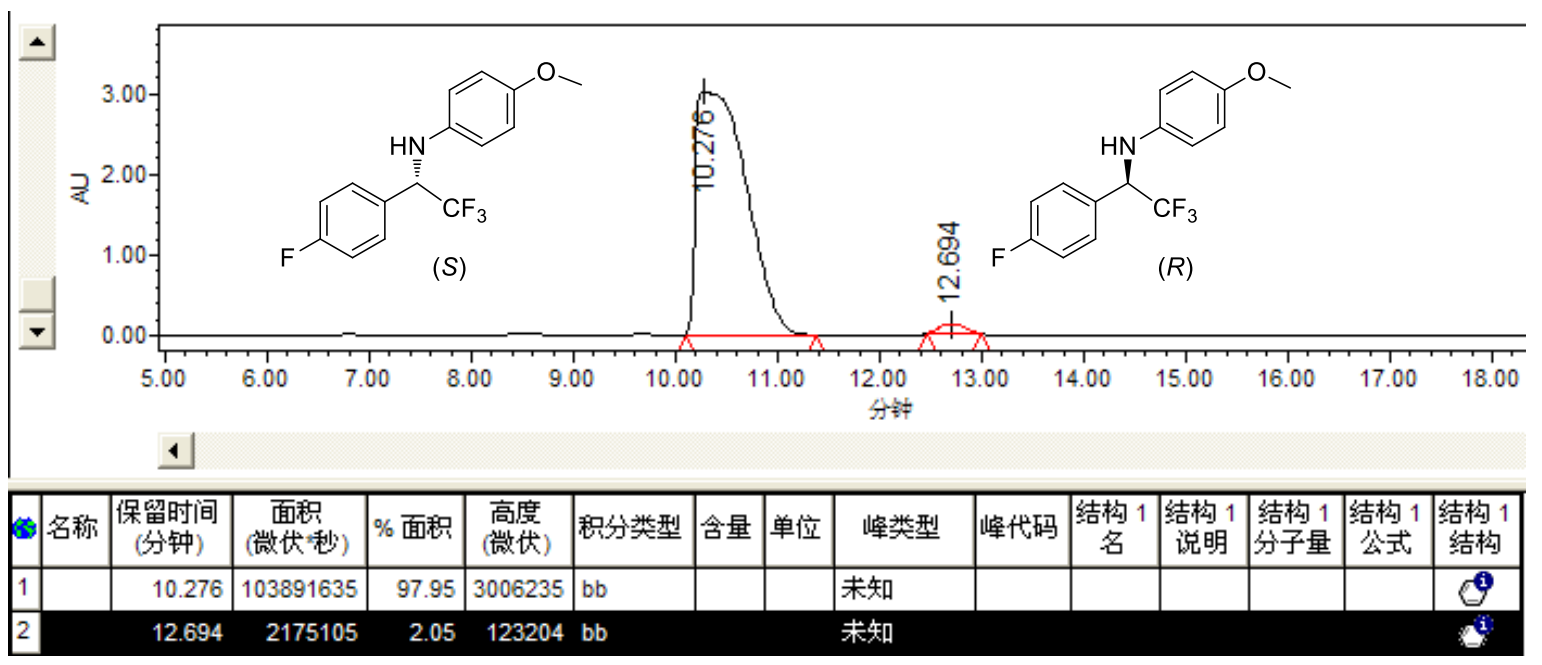

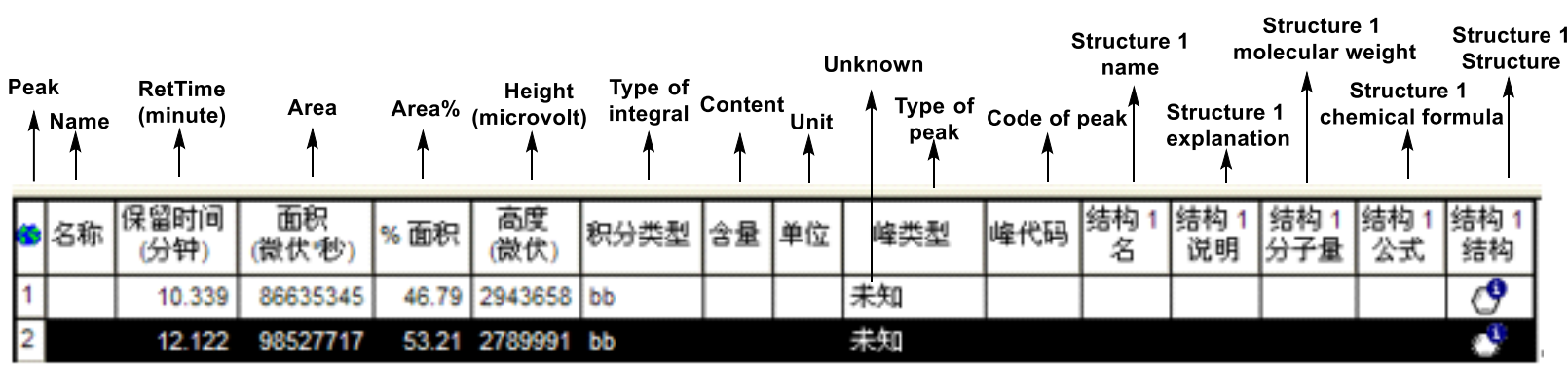


(S)-N-(1-(4-chlorophenyl)-2,2,2-trifluoroethyl)-4-methoxyaniline: (HPLC: Chiracel AD-H, detector: $254 \mathrm{~nm}$, elute: Hexanes/i-PrOH $=90 / 10$, flow rate: $0.8 \mathrm{~mL} / \mathrm{min}, 25^{\circ} \mathrm{C}, \mathrm{t}_{1}=11 . .0$ $\min (\mathrm{maj})$, $\mathrm{t}_{2}=14.5 \mathrm{~min}$ ).
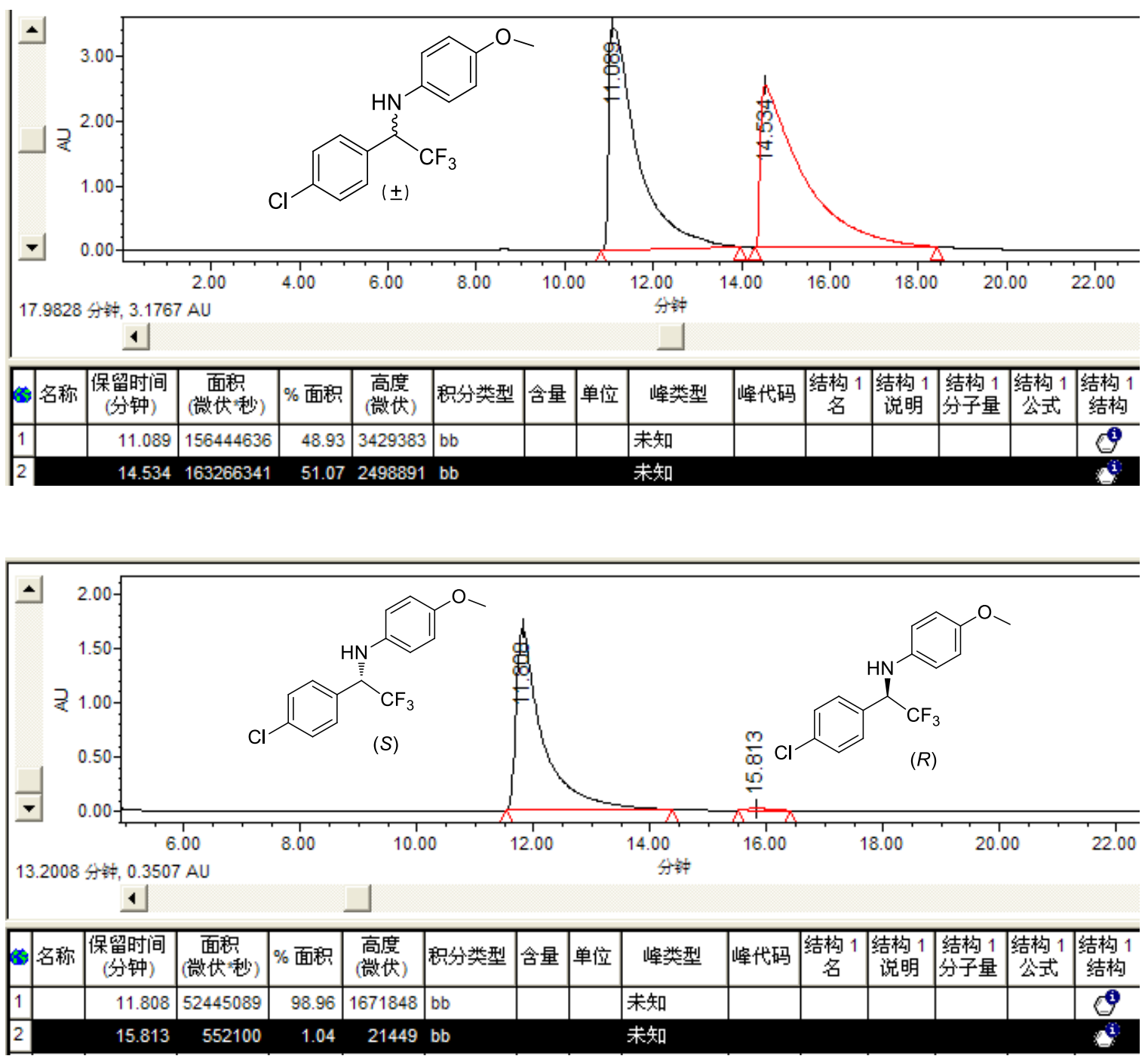

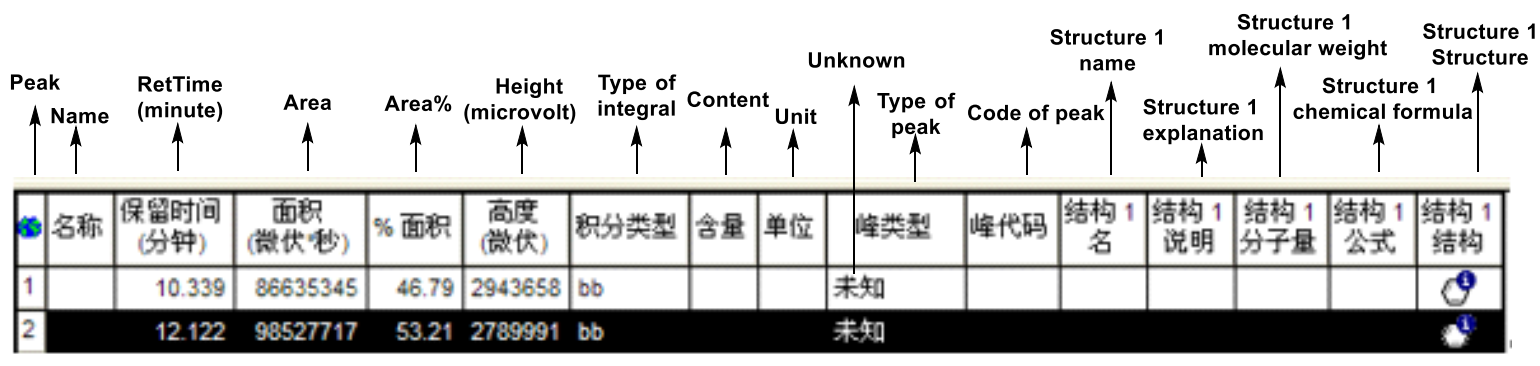


(S)-N-(1-(3-bromophenyl)-2,2,2-trifluoroethyl)-4-methoxyaniline: (HPLC: Chiracel OD-H, detector: $254 \mathrm{~nm}$, elute: Hexanes/i-PrOH $=95 / 5$, flow rate: $0.5 \mathrm{~mL} / \mathrm{min}, 25^{\circ} \mathrm{C}, \mathrm{t}_{1}=21.1 \mathrm{~min}$ (maj), t2 $=26.3 \mathrm{~min}$.)

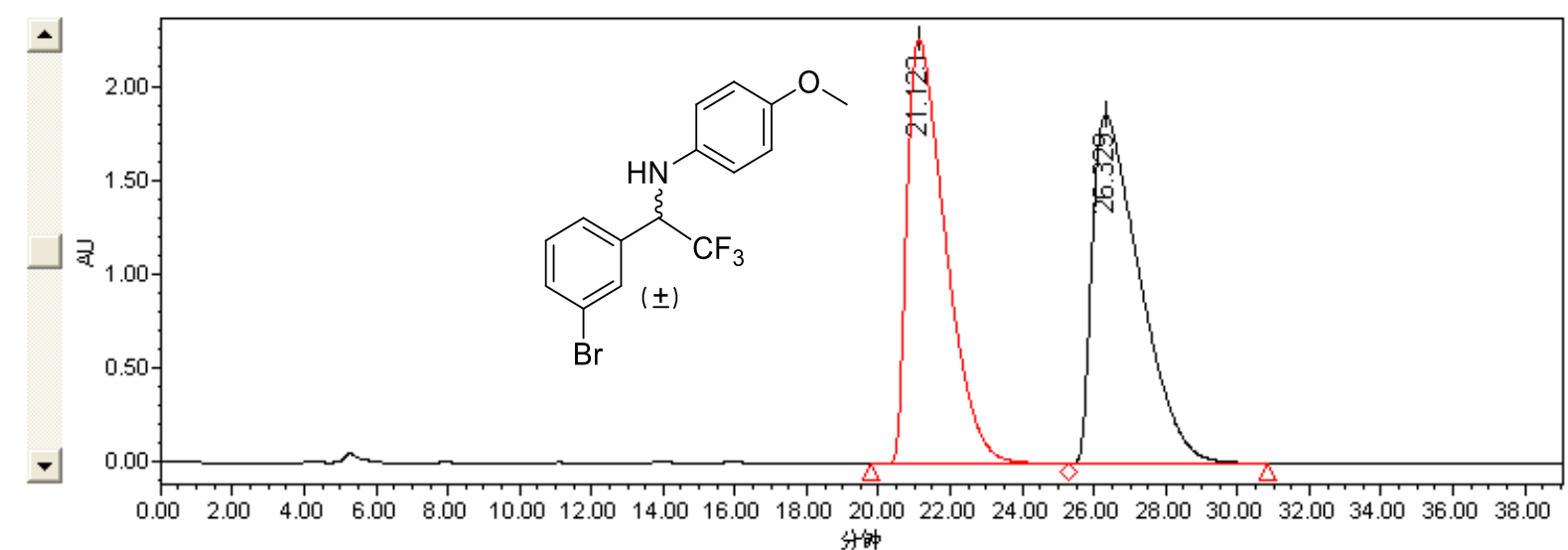

1

\begin{tabular}{|c|c|c|c|c|c|c|c|c|c|c|c|c|c|c|c|}
\hline 6 & 名称 & $\begin{array}{c}\text { 保留时间 } \\
\text { (分钟) }\end{array}$ & $\begin{array}{c}\text { 面积 } \\
\text { (凯代地) }\end{array}$ & $\%$ 面积 & $\begin{array}{c}\text { 高度 } \\
\text { (微代) }\end{array}$ & 积分类型 & 含量 & 单位 & 峖类型 & 峖代码 & \begin{tabular}{|c} 
结构 1 \\
名
\end{tabular} & \begin{tabular}{|c} 
结构 1 \\
说明
\end{tabular} & $\begin{array}{l}\text { 结构 } 1 \\
\text { 分子量 }\end{array}$ & $\begin{array}{l}\text { 结构 } 1 \\
\text { 公式 }\end{array}$ & $\begin{array}{l}\text { 结构 } 1 \\
\text { 结构 }\end{array}$ \\
\hline 1 & & 21.123 & 168097028 & 49.70 & 2253707 & BV & & & 未知 & & & & & & 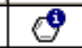 \\
\hline 2 & & 26.329 & 170094476 & 50.30 & 1852898 & VB & & & 末知 & & & & & & 6 \\
\hline
\end{tabular}

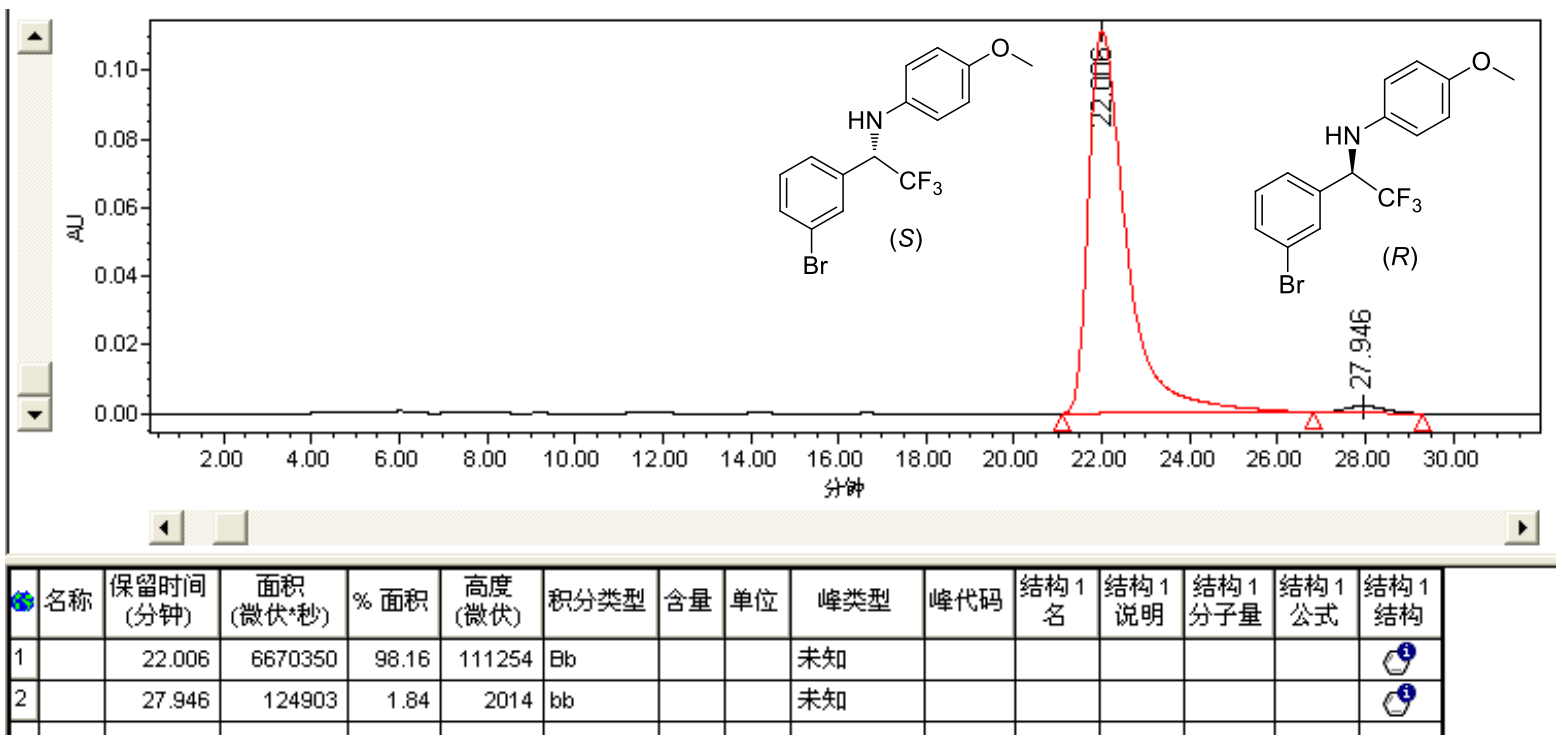

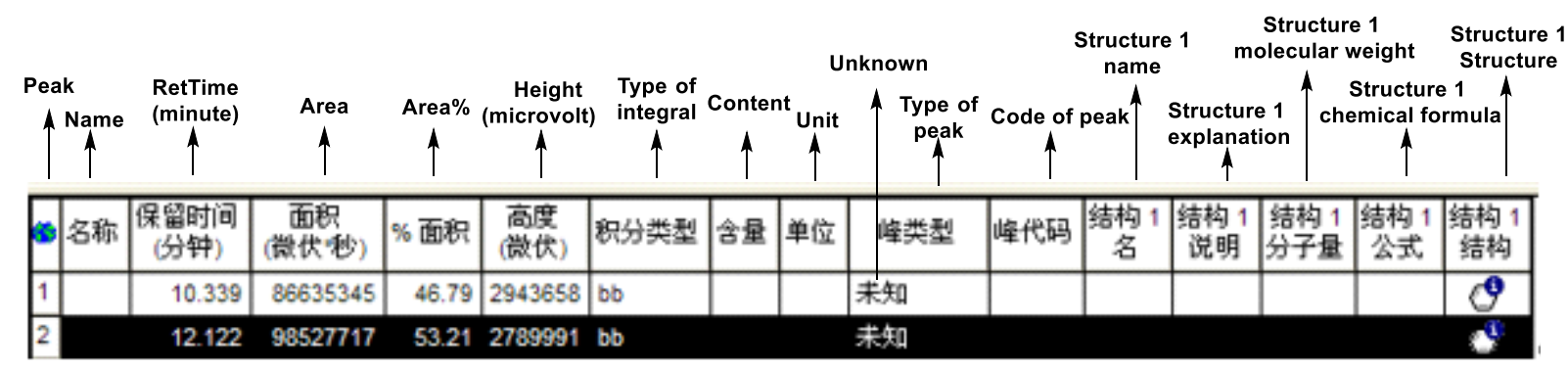


(S)-N-(1-(4-bromophenyl)-2,2,2-trifluoroethyl)-4-methoxyaniline: (HPLC: Chiracel OD-H, detector: $254 \mathrm{~nm}$, elute: Hexanes/i-PrOH = 95/5, flow rate: $0.5 \mathrm{~mL} / \mathrm{min}, 25^{\circ} \mathrm{C}$ ), $\mathrm{t}_{1}=12.2 \mathrm{~min}$ (maj), $\mathrm{t}_{2}=14.7 \mathrm{~min}$ )
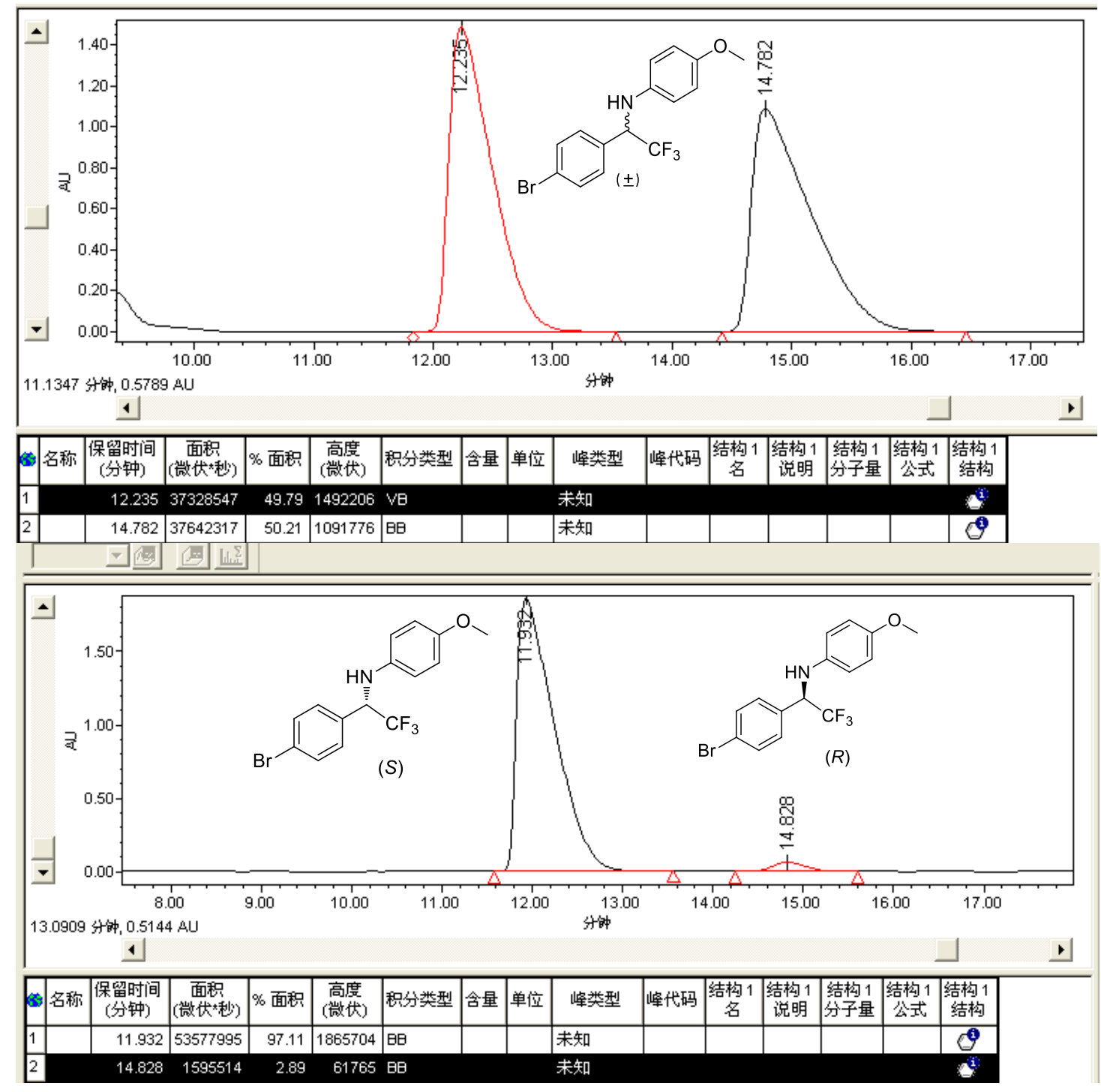

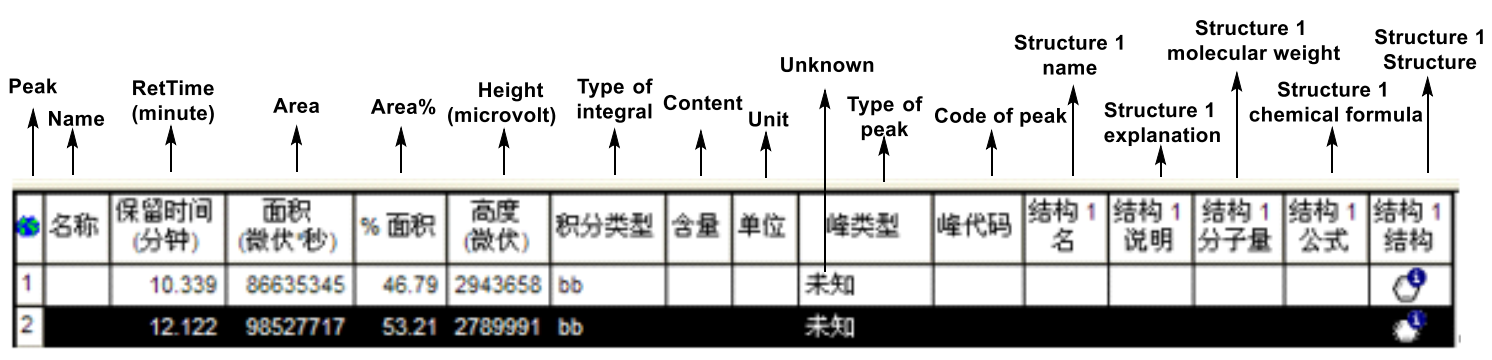


Chiracel OB-H, detector: $254 \mathrm{~nm}$, elute: Hexanes/i-PrOH = 90/10, flow rate: $0.5 \mathrm{~mL} / \mathrm{min}, 25$ ${ }^{\circ} \mathrm{C}, \mathrm{t}_{1}=21.1 \mathrm{~min}, \mathrm{t}_{2}=29.3 \mathrm{~min}$.)
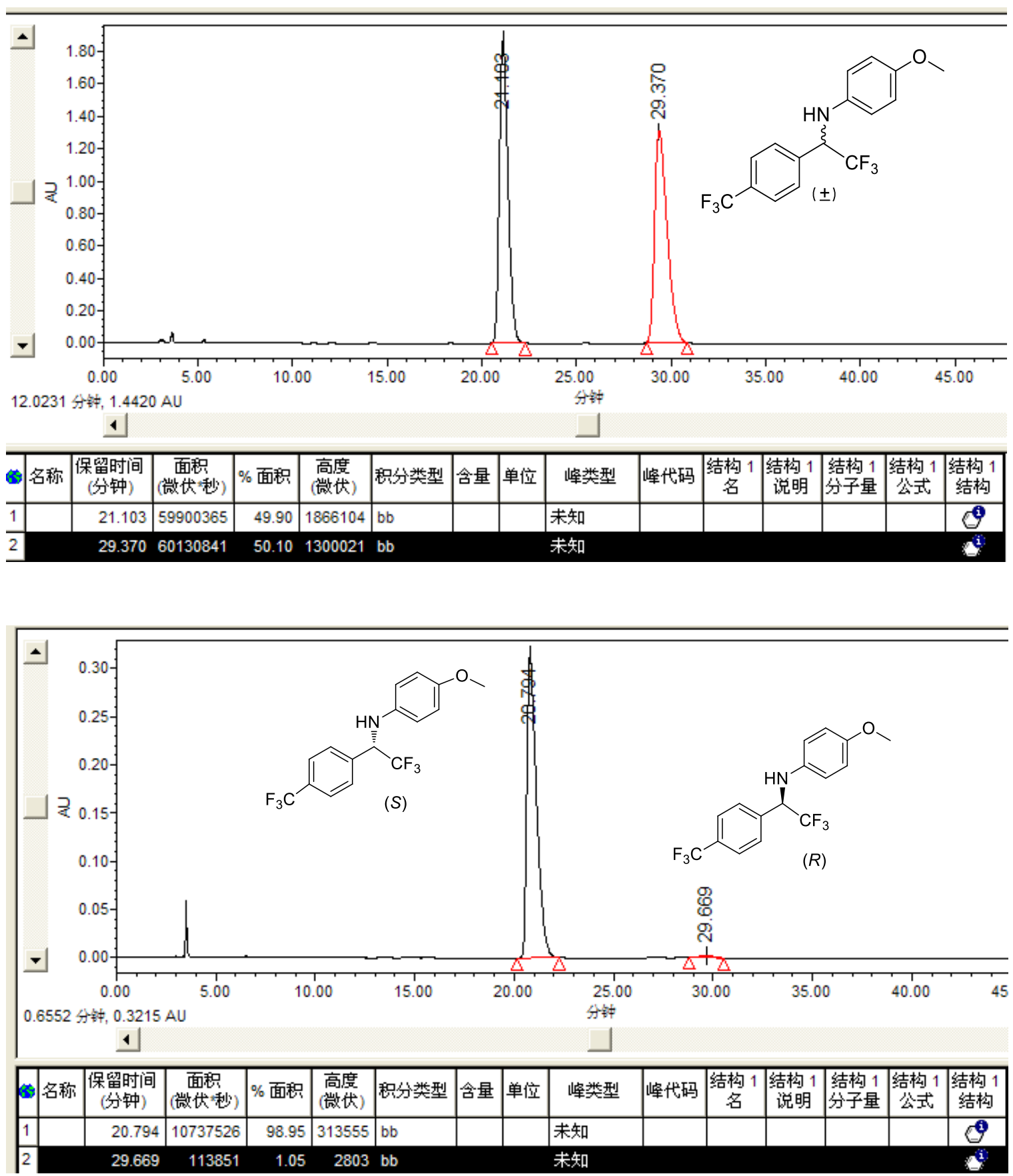

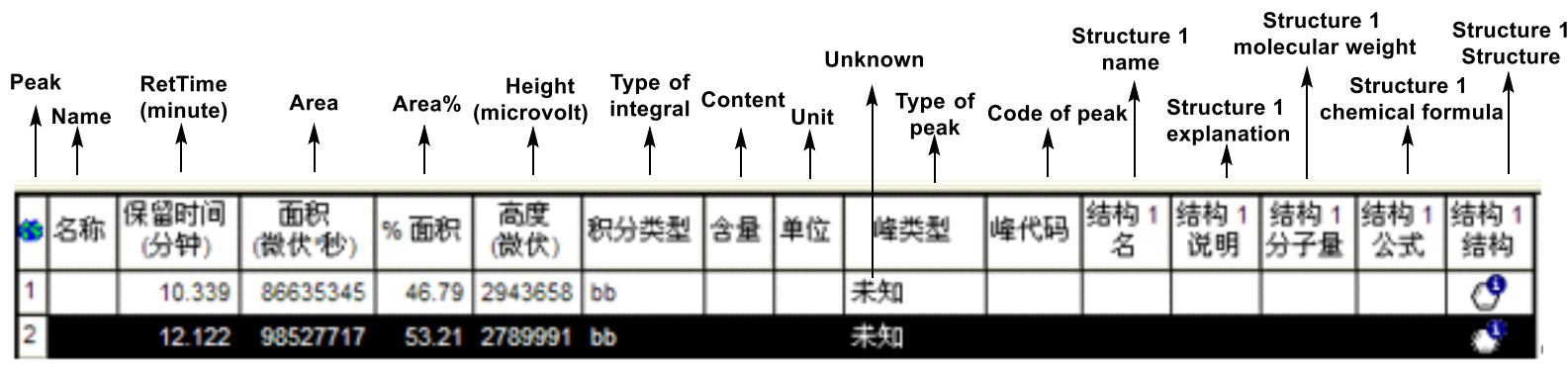


(S)-4-methoxy-N-(2,2,2-trifluoro-1-(p-tolyl)ethyl)aniline: (HPLC: Chiracel AD-H, detector: $254 \mathrm{~nm}$, elute: Hexanes/i-PrOH $=90 / 10$, flow rate: $0.8 \mathrm{~mL} / \mathrm{min}, 25^{\circ} \mathrm{C}, \mathrm{t}_{1}=7.7 \mathrm{~min}$ (maj), $\mathrm{t}_{2}=$ $10.4 \min$.
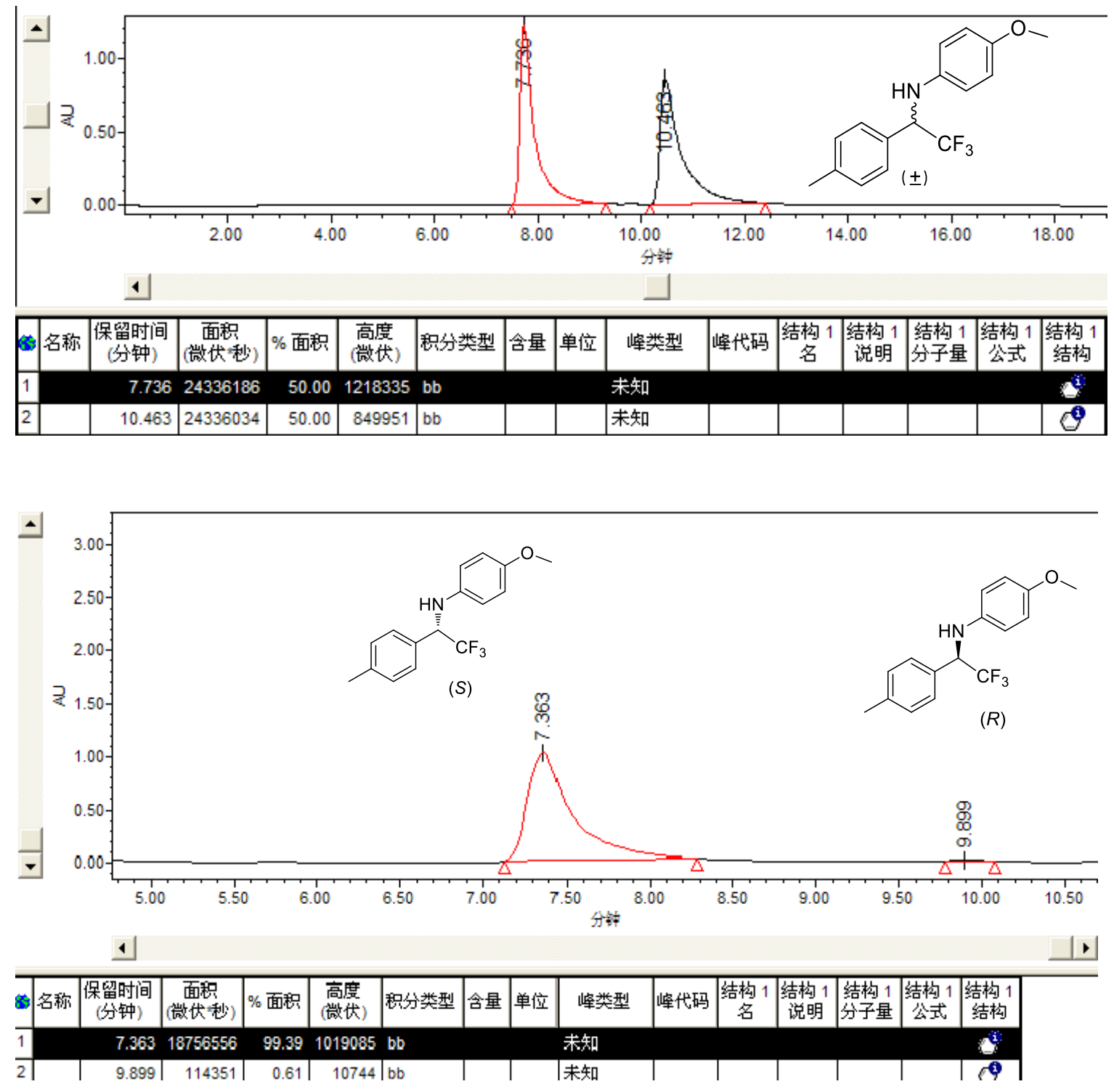

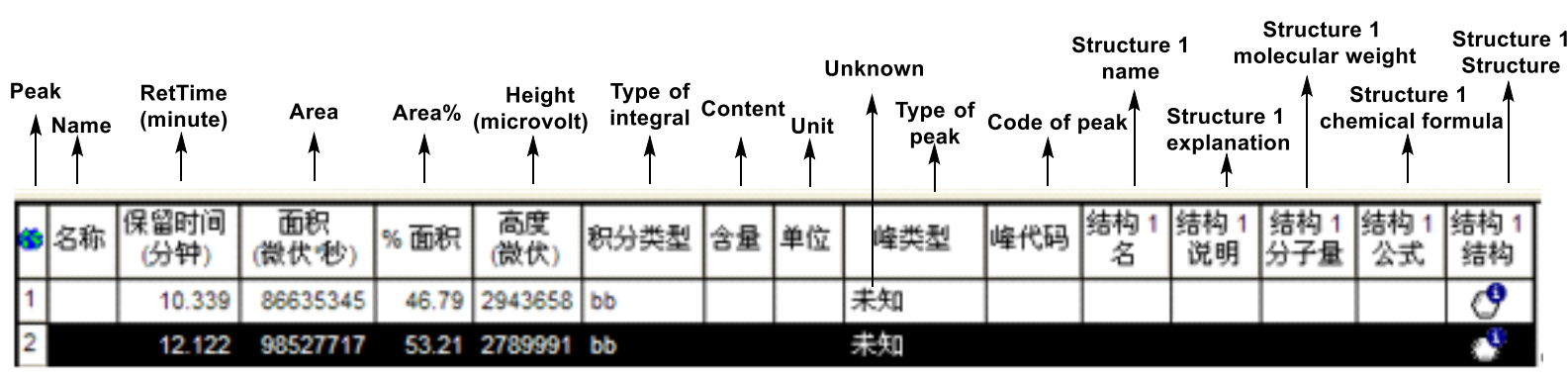


(S)-4-methoxy-N-(2,2,2-trifluoro-1-(4-methoxyphenyl)ethyl)aniline: (HPLC: Chiracel AD$\mathrm{H}$, detector: $254 \mathrm{~nm}$, elute: Hexanes/i-PrOH $=90 / 10$, flow rate: $0.8 \mathrm{~mL} / \mathrm{min}, 25^{\circ} \mathrm{C}$ ), $\mathrm{t}_{1}=11.1$ $\min (\mathrm{maj}), \mathrm{t}_{2}=16.0 \mathrm{~min}$.)
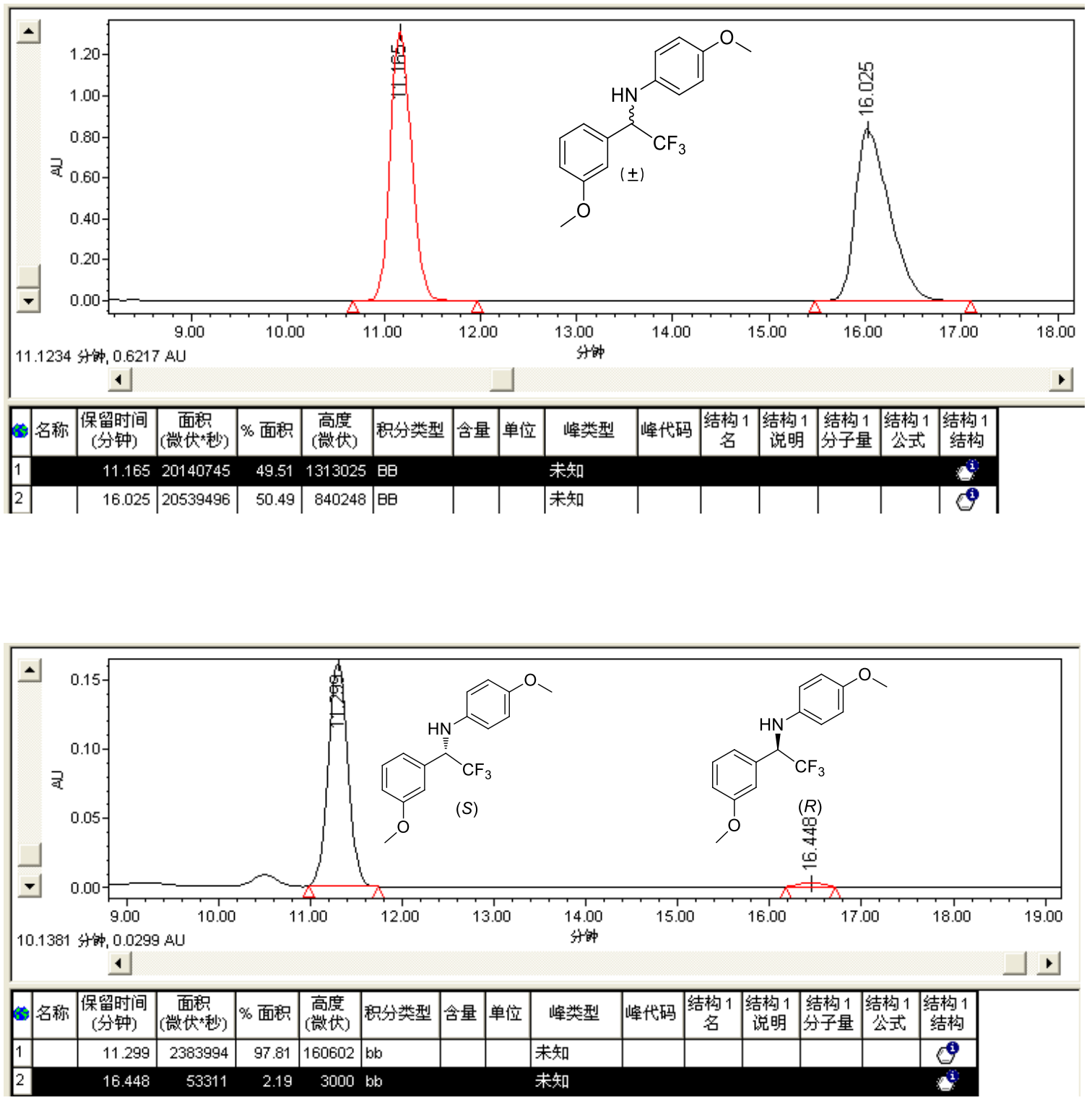

\begin{tabular}{|c|c|c|c|c|c|c|c|c|c|c|c|c|c|c|c|}
\hline \multirow{2}{*}{\multicolumn{2}{|c|}{$\begin{array}{l}\text { Peak } \\
\uparrow \uparrow\end{array}$}} & \multirow[b]{2}{*}{$\begin{array}{c}\text { RetTime } \\
\text { (minute) }\end{array}$} & \multirow[b]{2}{*}{$\stackrel{\text { Area }}{\uparrow}$} & \multirow[b]{2}{*}{$\stackrel{\text { Area } \%}{\uparrow}$} & \multirow[b]{2}{*}{$\begin{array}{c}\begin{array}{c}\text { Height } \\
\text { (microvolt) }\end{array} \\
\uparrow\end{array}$} & \multirow[b]{2}{*}{$\begin{array}{c}\text { Type of } \\
\text { integral }\end{array}$} & \multicolumn{3}{|c|}{ Unknown } & \multicolumn{3}{|c|}{$\begin{array}{c}\text { Structure } 1 \\
\text { name }\end{array}$} & \multicolumn{2}{|c|}{$\begin{array}{l}\text { Structure } 1 \\
\text { molecular weight }\end{array}$} & \multirow[t]{2}{*}{$\begin{array}{l}\text { Structure } \\
\text { Structure }\end{array}$} \\
\hline & & & & & & & Sonten & Unit & $\left.\uparrow \begin{array}{c}\text { Type of } \\
\text { peak }\end{array}\right\}$ & Code of & peak $\uparrow$ & $\begin{array}{l}\text { Structure } \\
\text { explanati }\end{array}$ & $\begin{array}{l}\text { e } 1 \\
\text { tion }\end{array}$ & $\begin{array}{c}\text { Structure } \\
\text { hemical fo }\end{array}$ & \\
\hline 3 & 名称 & $\begin{array}{l}\text { 保留时间 } \\
\text { (分钟) }\end{array}$ & 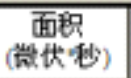 & \% 面积 & $\begin{array}{l}\text { 高度 } \\
\text { (微伏) }\end{array}$ & 积分类型 & 상은 & 单位 & 峰类型 & 峰代码 & $\begin{array}{c}\text { 结构 } \\
\text { 名 }\end{array}$ & $\mid \begin{array}{c}\text { 结构 1 } \\
\text { 说明 }\end{array}$ & $\begin{array}{l}\text { 结构 } 1 \\
\text { 分子量 }\end{array}$ & \begin{tabular}{|l|l|}
1 & 结构 1 \\
公式
\end{tabular} & \begin{tabular}{|l} 
结构 1 \\
结构
\end{tabular} \\
\hline 1 & & 10.339 & 86635345 & 46.79 & 2943658 & bo & & & 末知 & & & & & & 9 \\
\hline 2 & & 12.122 & 98527717 & 53.21 & 2789991 & bb & & & 未知 & & & & & & di \\
\hline
\end{tabular}


(S)-4-methoxy-N-(2,2,2-trifluoro-1-(thiophen-2-yl)ethyl)aniline: (HPLC: Chiracel AD-H, detector: $254 \mathrm{~nm}$, elute: Hexanes/i-PrOH $=97 / 3$, flow rate: $1.0 \mathrm{~mL} / \mathrm{min}, 25^{\circ} \mathrm{C}$ ), $\mathrm{t}_{1}=10.3 \mathrm{~min}$ (maj), $\mathrm{t}_{2}=12.6 \mathrm{~min}$.)

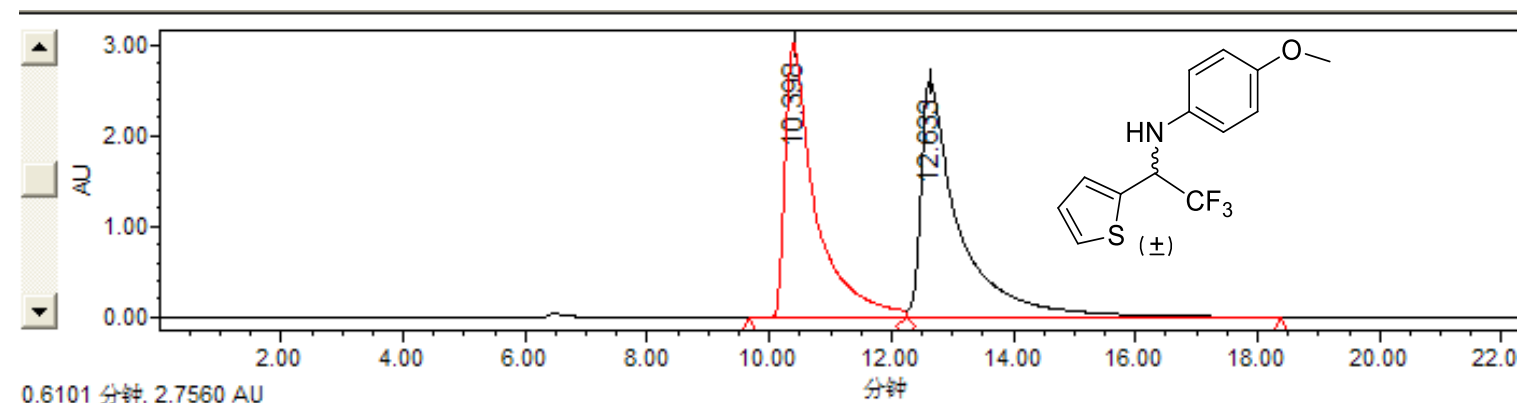
0.6101 分䖵, $2.7560 \mathrm{AU}$

4

\begin{tabular}{|c|c|c|c|c|c|c|c|c|c|c|c|c|c|c|c|}
\hline 5 & 名称 & \begin{tabular}{|c} 
保留时间 \\
(分钟)
\end{tabular} & $\begin{array}{c}\text { 面积 } \\
\text { (微伏秒) }\end{array}$ & \% 面积 & $\begin{array}{l}\text { 高席 } \\
\text { (微伏) }\end{array}$ & 积分类型 & 含量 & 单位 & 㛡类型 & 峖代码 & \begin{tabular}{|c} 
结构 1 \\
名
\end{tabular} & \begin{tabular}{|c|} 
结构 1 \\
说明
\end{tabular} & \begin{tabular}{|l} 
结构 1 \\
分子量
\end{tabular} & \begin{tabular}{|l} 
结构 1 \\
公式
\end{tabular} & \begin{tabular}{|l} 
结构 1 \\
结构
\end{tabular} \\
\hline 1 & & 10.398 & 103356677 & 47.70 & 3012952 & BV & & & 未知 & & & & & & 8 \\
\hline 2 & & 12.633 & 113324009 & 52.30 & 2599200 & VB & & & 未知 & & & & & & (3) \\
\hline
\end{tabular}

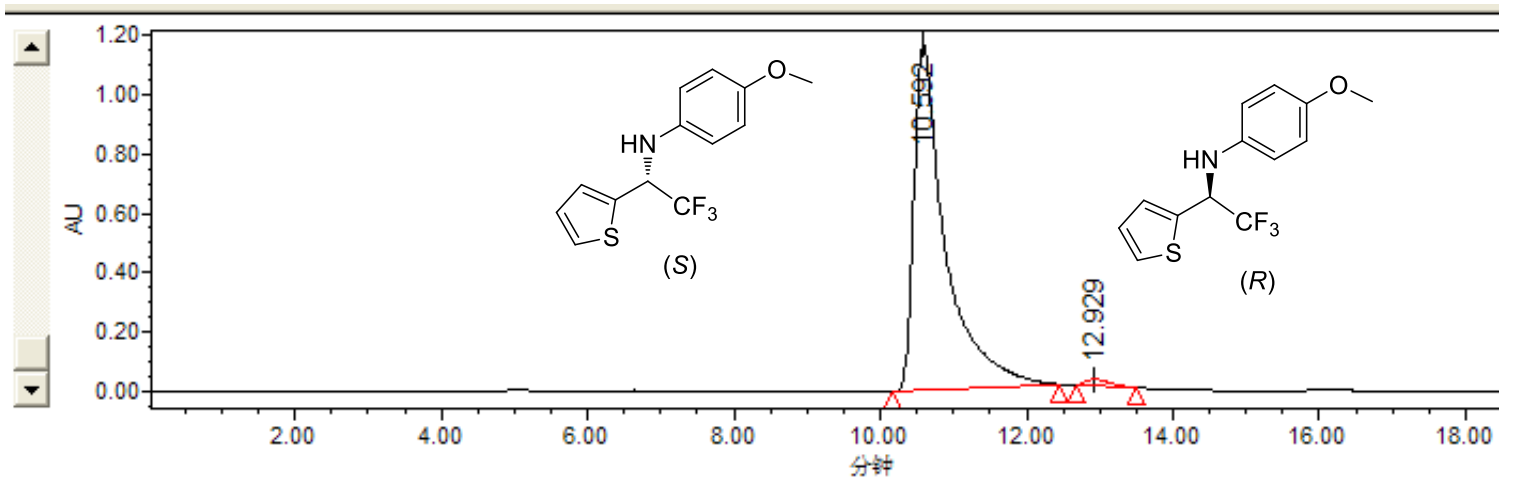

1. $\perp$

\begin{tabular}{|c|c|c|c|c|c|c|c|c|c|c|c|c|c|c|}
\hline 名称 & \begin{tabular}{|c} 
保留时间 \\
(分钟)
\end{tabular} & $\begin{array}{c}\text { 面积 } \\
\text { (微伏秒) }\end{array}$ & \% 面积 & $\begin{array}{c}\text { 高庶 } \\
\text { (微伏) }\end{array}$ & 积分类型 & 含量 & 单位 & 㷨类型 & 峖代码 & $\begin{array}{c}\text { 结构 } 1 \\
\text { 名 }\end{array}$ & \begin{tabular}{|} 
结构 1 \\
说明
\end{tabular} & $\begin{array}{l}\text { 结构 } 1 \\
\text { 分子量 }\end{array}$ & $\begin{array}{l}\text { 结构 } 1 \\
\text { 公式 }\end{array}$ & \begin{tabular}{|l} 
结构 1 \\
结构
\end{tabular} \\
\hline 1 & 10.592 & 34695326 & 98.61 & 1159557 & bb & & & 未知 & & & & & & (9) \\
\hline & 12.929 & 488974 & 1.39 & 21770 & $\mathrm{bb}$ & & & 未知 & & & & & & 8 \\
\hline
\end{tabular}

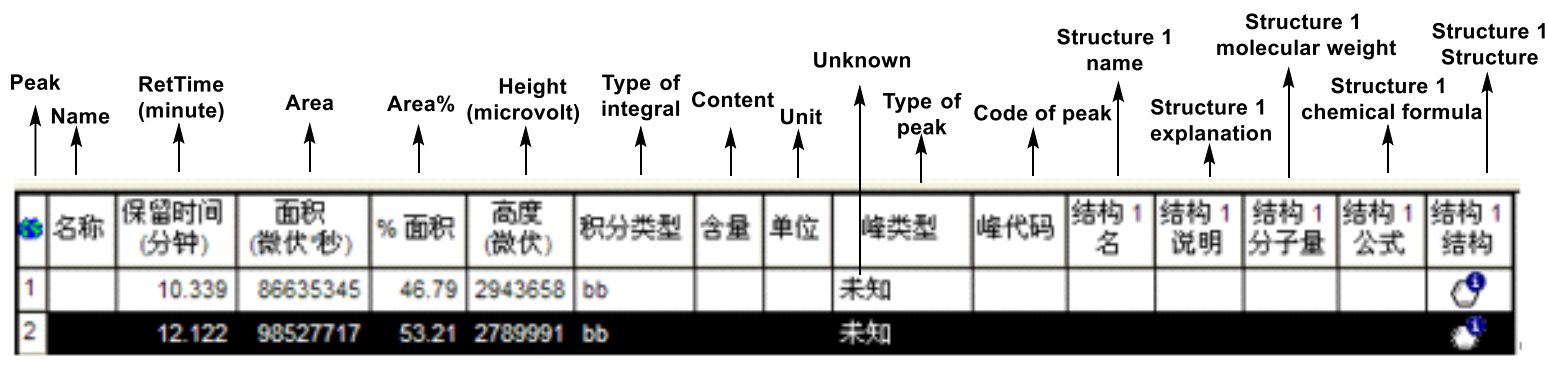


(S)-4-methyl-N-(2,2,2-trifluoro-1-phenylethyl)aniline: (HPLC: Chiracel OD-H, detector: $254 \mathrm{~nm}$, elute: Hexanes/i-PrOH $=97 / 3$, flow rate: $1.0 \mathrm{~mL} / \mathrm{min}, 25^{\circ} \mathrm{C}, \mathrm{t}_{1}=6.0 \mathrm{~min}$ (maj), $\mathrm{t}_{2}=$ 8.3 min.)

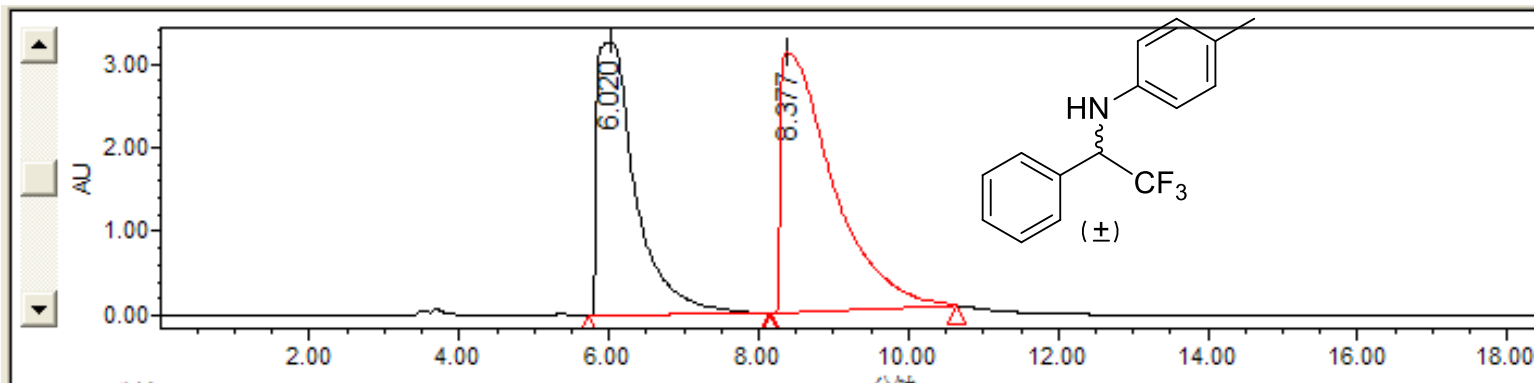
10.0019 分钎, $1.4284 \mathrm{AU}$

1

\begin{tabular}{|c|c|c|c|c|c|c|c|c|c|c|c|c|c|c|c|}
\hline 8 & 名称 & $\begin{array}{c}\text { 保留时间 } \\
\text { (分钟) }\end{array}$ & $\begin{array}{c}\text { 面积 } \\
\text { (凯代利) }\end{array}$ & $\%$ 面积 & $\begin{array}{l}\text { 高度 } \\
\text { (溦伏) }\end{array}$ & 积分类型 & 含量 & 单位 & 㛡类型 & 姩代码 & $\begin{array}{c}\text { 结构 } 1 \\
\text { 名 }\end{array}$ & $\begin{array}{c}\text { 结构 } 1 \\
\text { 说明 }\end{array}$ & $\begin{array}{l}\text { 结构 } 1 \\
\text { 分子量 }\end{array}$ & $\begin{array}{l}\text { 结构 } 1 \\
\text { 公式 }\end{array}$ & $\begin{array}{c}\text { 结构 } 1 \\
\text { 结构 }\end{array}$ \\
\hline 1 & & 6.020 & 115344432 & 43.48 & 3260336 & bb & & & 未知 & & & & & & $\theta$ \\
\hline
\end{tabular}

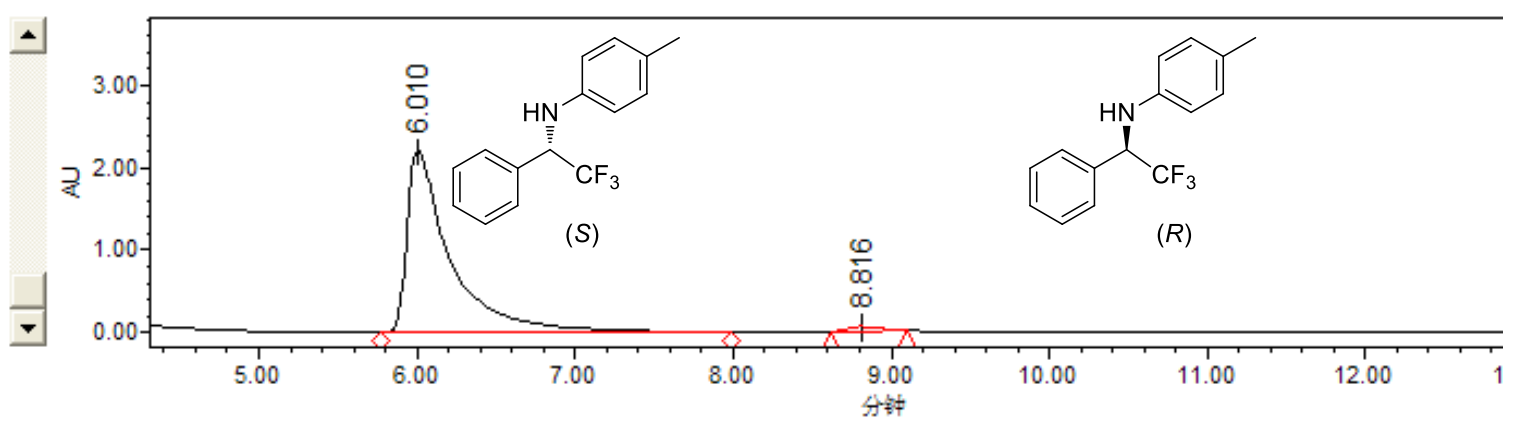

1

\begin{tabular}{|c|c|c|c|c|c|c|c|c|c|c|c|c|c|c|c|}
\hline$\Leftrightarrow$ & 名称 & \begin{tabular}{|c|} 
保留时间 \\
(分钟)
\end{tabular} & $\begin{array}{c}\text { 面积 } \\
\text { (钽代利) }\end{array}$ & \% 面积 & $\begin{array}{l}\text { 高庶 } \\
\text { (凯伏) }\end{array}$ & 积分类型 & 含量 & 单位 & 聺类型 & 㷨代码 & $\begin{array}{c}\text { 结构 } 1 \\
\text { 名 }\end{array}$ & \begin{tabular}{|c|} 
结构 1 \\
说明
\end{tabular} & $\begin{array}{l}\text { 结构 } 1 \\
\text { 分子量 }\end{array}$ & \begin{tabular}{|c|} 
结构 1 \\
公式
\end{tabular} & $\begin{array}{c}\text { 结构 } 1 \\
\text { 结构 }\end{array}$ \\
\hline 1 & & 6.010 & 42785922 & 98.05 & 2224000 & v & & & 未知 & & & & & & 8 \\
\hline 2 & & 8.816 & 851079 & 1.95 & 58463 & bb & & & 未知 & & & & & & \\
\hline
\end{tabular}

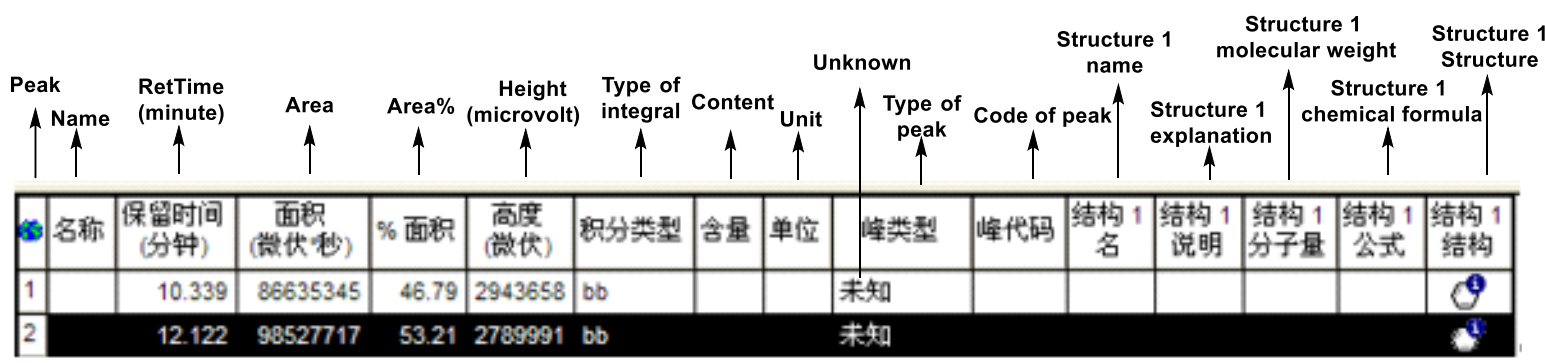


(S)-4-methyl-N-(2,2,2-trifluoro-1-(4-fluorophenyl)ethyl)aniline: (HPLC: Chiracel OD-H, elute: Hexanes/i-PrOH $=97 / 3$, detector: $254 \mathrm{~nm}$, flow rate: $1.0 \mathrm{~mL} / \mathrm{min}, 25^{\circ} \mathrm{C}, \mathrm{t}_{1}=11.5 \mathrm{~min}$ (maj), $\mathrm{t}_{2}=14.7 \mathrm{~min}$.)

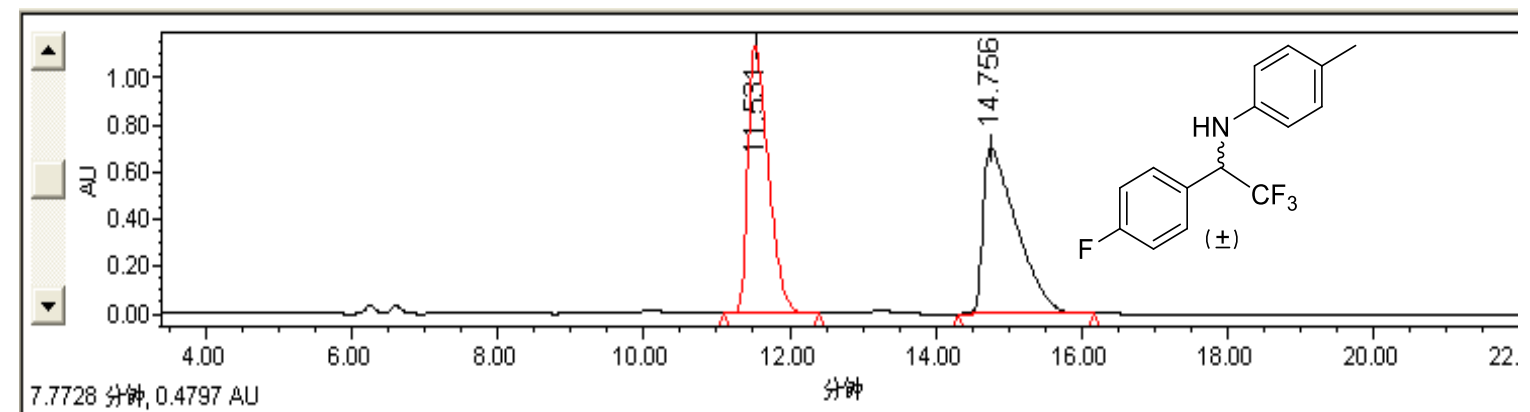
7.7728 出部, 0.4797 AU

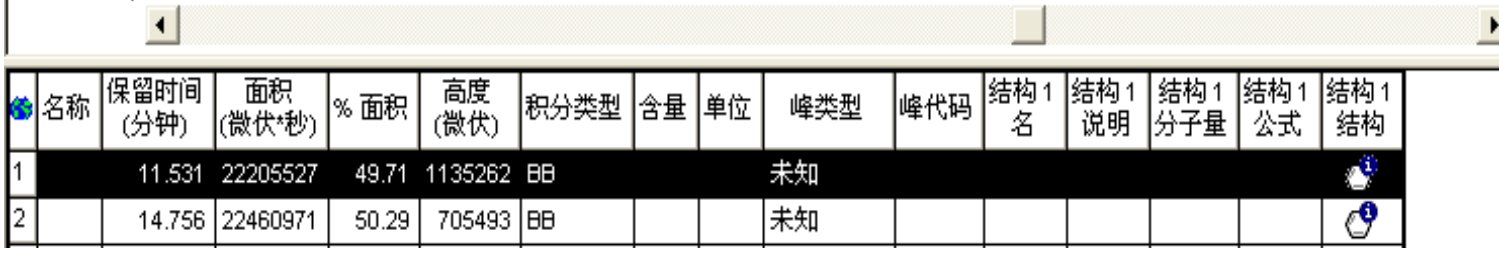

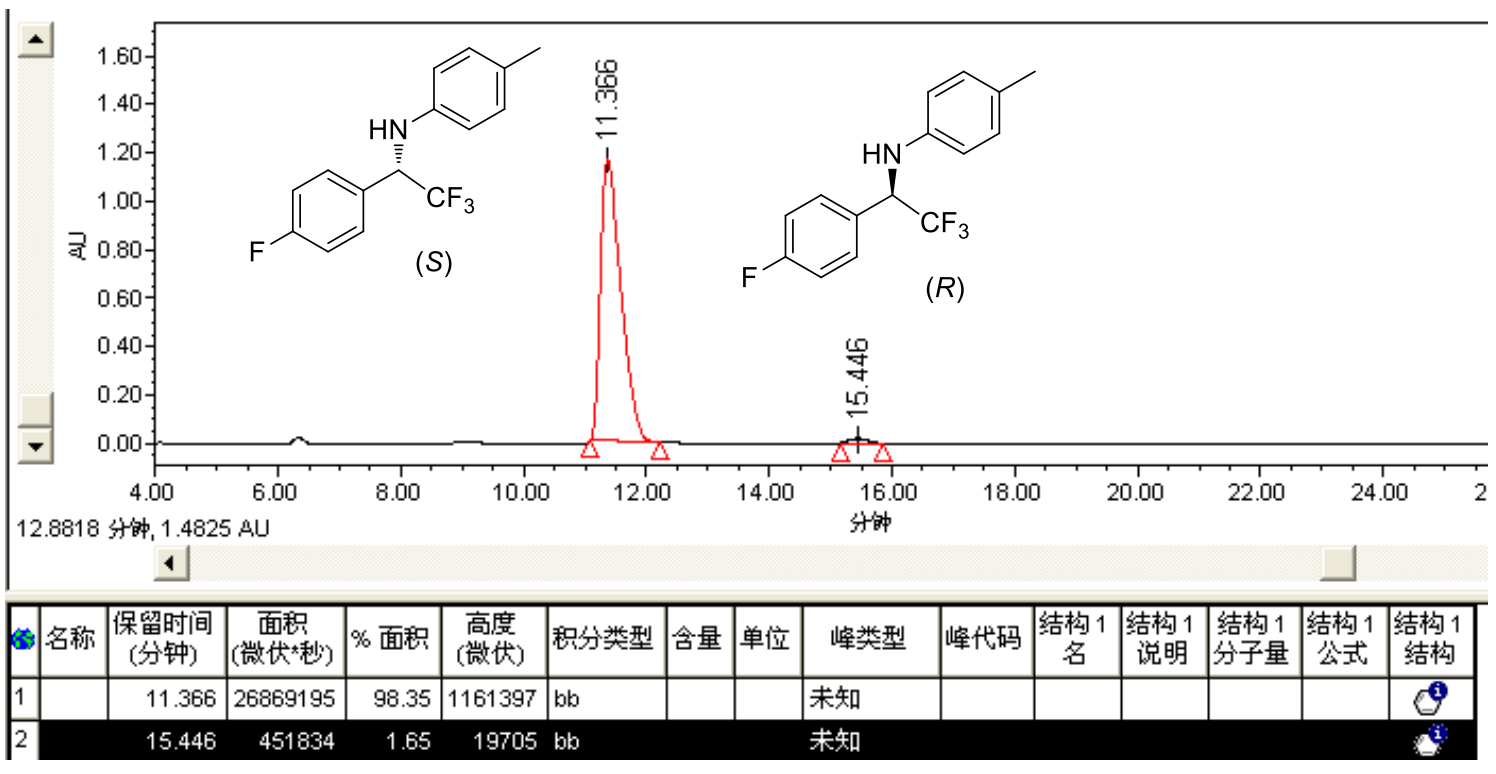

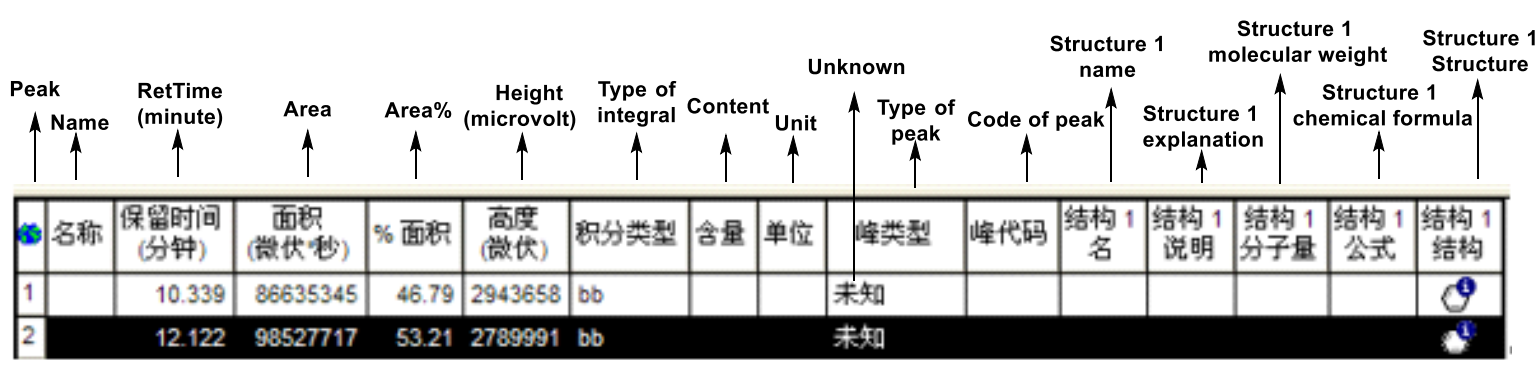


(S)-N-(1-(4-bromophenyl)-2,2,2-trifluoroethyl)-4-methoxyaniline: (HPLC: Chiracel OD-H, detector: $254 \mathrm{~nm}$, elute: Hexanes/i-PrOH = 95/5, flow rate: $\left.1.0 \mathrm{~mL} / \mathrm{min}, 25^{\circ} \mathrm{C}\right), \mathrm{t}_{1}=11.1 \mathrm{~min}$ (maj), t2 $=15.8 \mathrm{~min}$.)
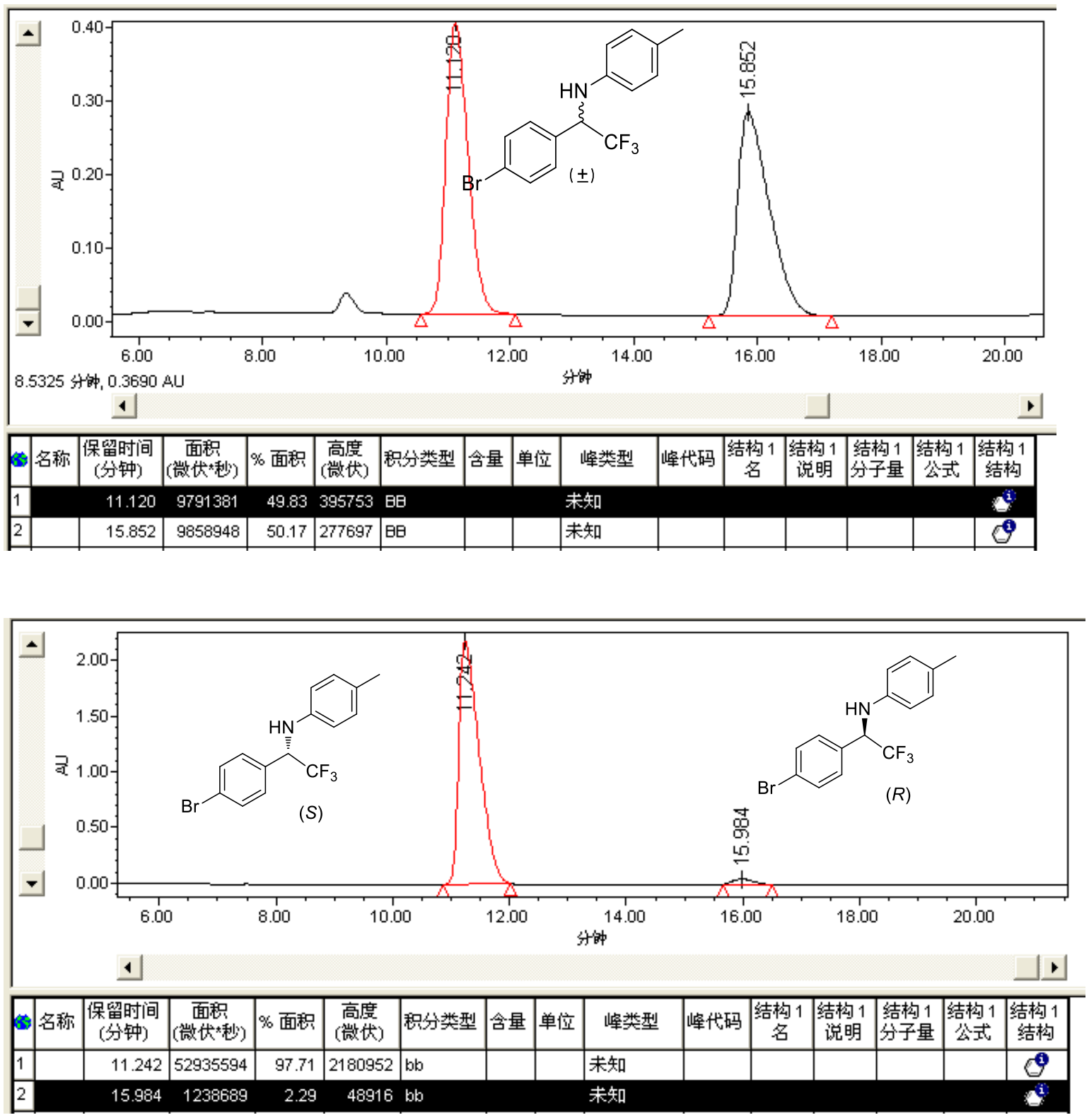

\begin{tabular}{|c|c|c|c|c|c|c|c|c|c|c|c|c|c|c|c|c|}
\hline \multicolumn{2}{|c|}{$\uparrow \uparrow$} & $\begin{array}{l}\text { RetTime } \\
\text { (minute) }\end{array}$ & $\stackrel{\text { Area }}{\uparrow}$ & $\stackrel{\text { Area } \%}{\uparrow}$ & $\begin{array}{c}\begin{array}{c}\text { Height } \\
\text { (microvolt) }\end{array} \\
\uparrow\end{array}$ & $\begin{array}{l}\text { Type of } \\
\text { integral }\end{array}$ & \multicolumn{3}{|c|}{ Unknown } & \multicolumn{3}{|c|}{$\begin{array}{l}\text { Structure } 1 \\
\text { name }\end{array}$} & \multicolumn{3}{|c|}{$\begin{array}{c}\text { Structure } 1 \\
\text { molecular weight }\end{array}$} & $\begin{array}{l}\text { Structure } \\
\text { Structure } \\
1\end{array}$ \\
\hline 3 & 名称 & $\begin{array}{c}\text { 保舅时间 } \\
\text { (分钟) }\end{array}$ & $\begin{array}{c}\text { 面帜 } \\
\text { (敞仗㠺) }\end{array}$ & \% 面积 & $\begin{array}{l}\text { 高度 } \\
\text { (敞伏) }\end{array}$ & 积分劵型 & 삼量 & 单位 & 捗类型 & 嶂代码 & $\begin{array}{c}\text { 结构 } 1 \\
\text { 名 }\end{array}$ & \begin{tabular}{|c} 
结构 1 \\
说明
\end{tabular} & $\begin{array}{l}\text { 结构 } \\
\text { 分子 }\end{array}$ & $\begin{array}{l}1 \\
\text { 量 }\end{array}$ & $\begin{array}{l}\text { 结构 } 1 \\
\text { 公式 }\end{array}$ & \begin{tabular}{|c|} 
结构 1 \\
结构
\end{tabular} \\
\hline 2 & & 12.122 & 98527717 & 53.21 & 2789991 & bb & & & 米知 & & & & & & & di: \\
\hline
\end{tabular}


(S)-N-(2,2,2-trifluoro-1-phenylethyl)aniline: (HPLC: Chiracel AD-H, detector: $254 \mathrm{~nm}$, elute: Hexanes/i-PrOH = 95/5, flow rate: $1.0 \mathrm{~mL} / \mathrm{min}, 25^{\circ} \mathrm{C}$ ), $\mathrm{t}_{1}=5.7 \mathrm{~min}$ (maj), $\mathrm{t}_{2}=7.2 \mathrm{~min}$.)
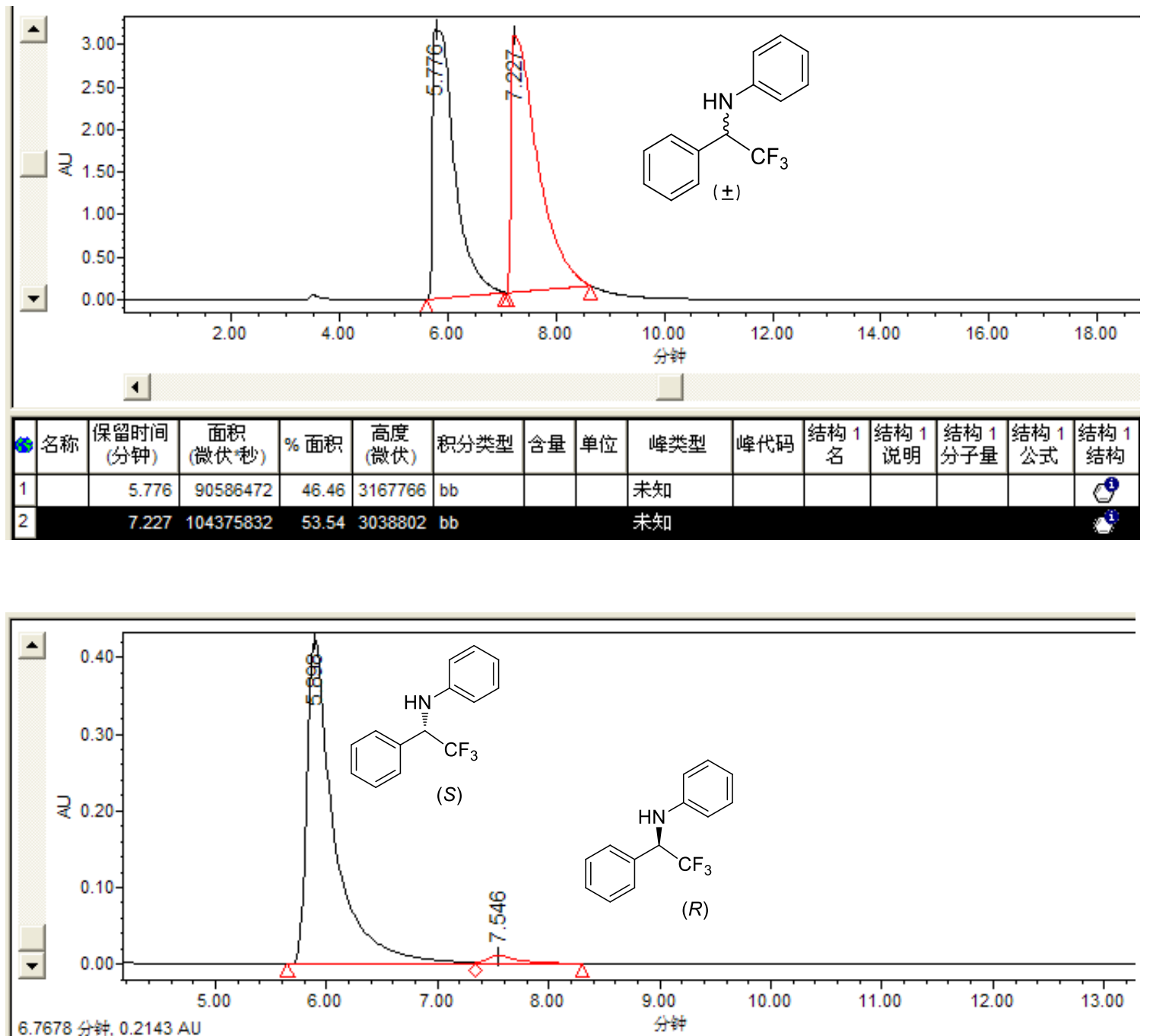

6.7678 分神, $0.2143 \mathrm{AU}$

1

\begin{tabular}{|c|c|c|c|c|c|c|c|c|c|c|c|c|c|c|c|}
\hline 8 & 名称 & $\begin{array}{c}\text { 保留时间 } \\
\text { (分钟) }\end{array}$ & $\begin{array}{c}\text { 面积 } \\
\text { (微伏“粆) }\end{array}$ & \% 面积 & $\begin{array}{c}\text { 高庶 } \\
\text { (微伏) }\end{array}$ & 积分类型 & 含量 & 单位 & 姩类型 & 峰代码 & $\begin{array}{l}\text { 结构 } 1 \\
\text { 名 }\end{array}$ & $\begin{array}{l}\text { 结构 } 1 \\
\text { 说明 }\end{array}$ & \begin{tabular}{|l} 
结构 1 \\
分子量
\end{tabular} & $\begin{array}{l}\text { 结构 } 1 \\
\text { 公式 }\end{array}$ & \begin{tabular}{|l} 
结构 1 \\
结构
\end{tabular} \\
\hline 1 & & 5.898 & 7274204 & 97.19 & 421983 & BV & & & 未知 & & & & & & (3) \\
\hline & & 7.546 & 210522 & 2.81 & 10660 & VB & & & 未知 & & & & & & i. \\
\hline
\end{tabular}

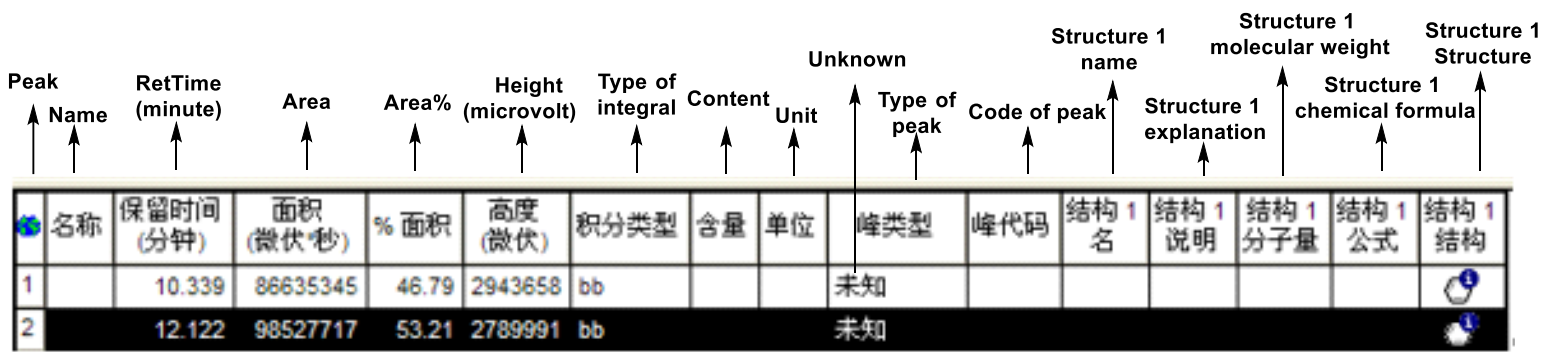


(S)-4-chloro-N-(2,2,2-trifluoro-1-phenylethyl)aniline: (HPLC: Chiracel OD-H, detector: $254 \mathrm{~nm}$, elute: Hexanes/i-PrOH $=95 / 5$, flow rate: $0.5 \mathrm{~mL} / \mathrm{min}, 25^{\circ} \mathrm{C}, \mathrm{t}_{1}=7.2 \mathrm{~min}$ (maj), $\mathrm{t}_{2}=$ 8.6 min.)
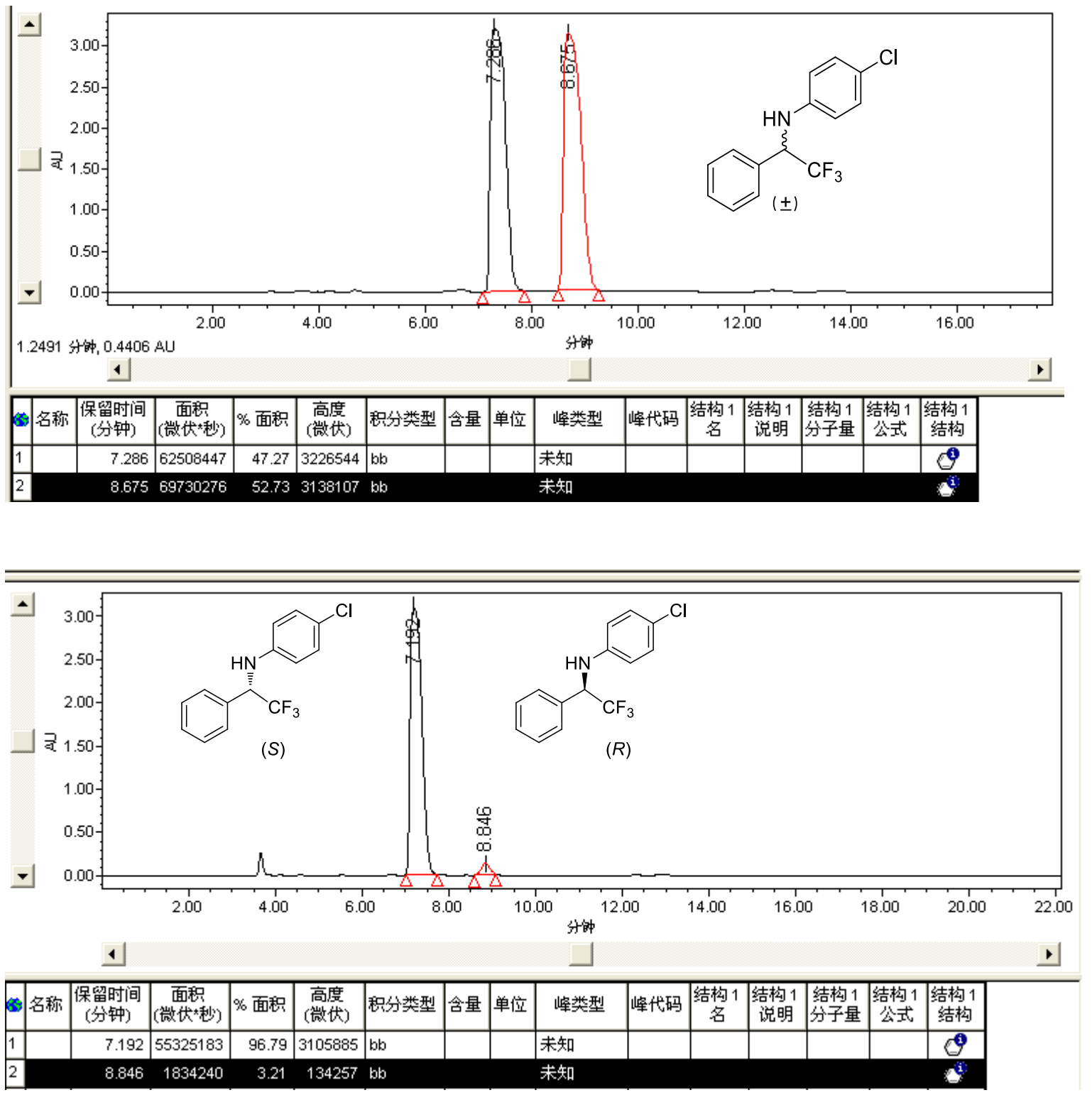

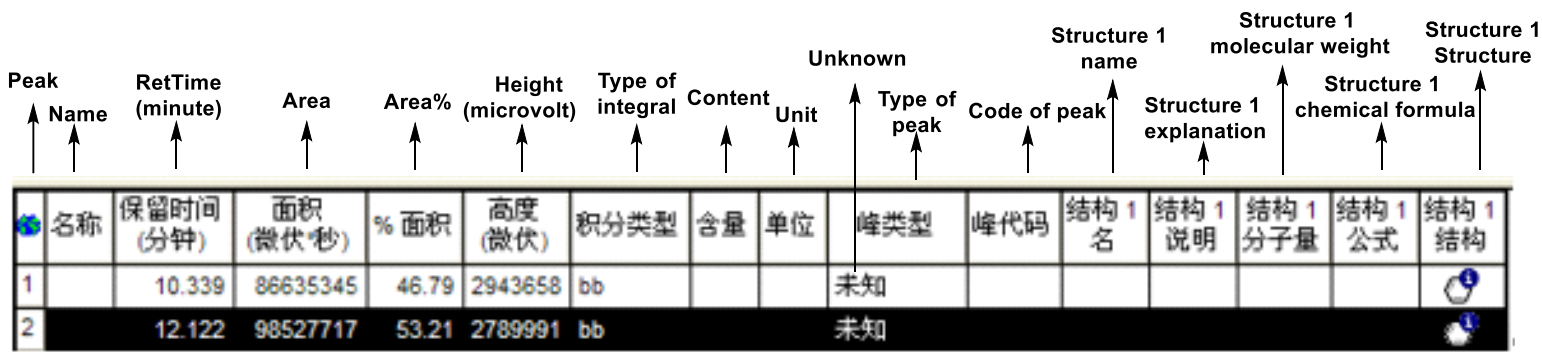


(S)-N-(2,2,2-trifluoro-1-phenylethyl)naphthalen-2-amine: (HPLC: Chiracel OD-H, detector: $254 \mathrm{~nm}$, elute: Hexanes/i-PrOH = 95/5, flow rate: $0.5 \mathrm{~mL} / \mathrm{min}, 25^{\circ} \mathrm{C}$ ), $\mathrm{t}_{1}=8.7 \mathrm{~min}$ (maj), $\mathrm{t}_{2}=$ 13.5 min.)
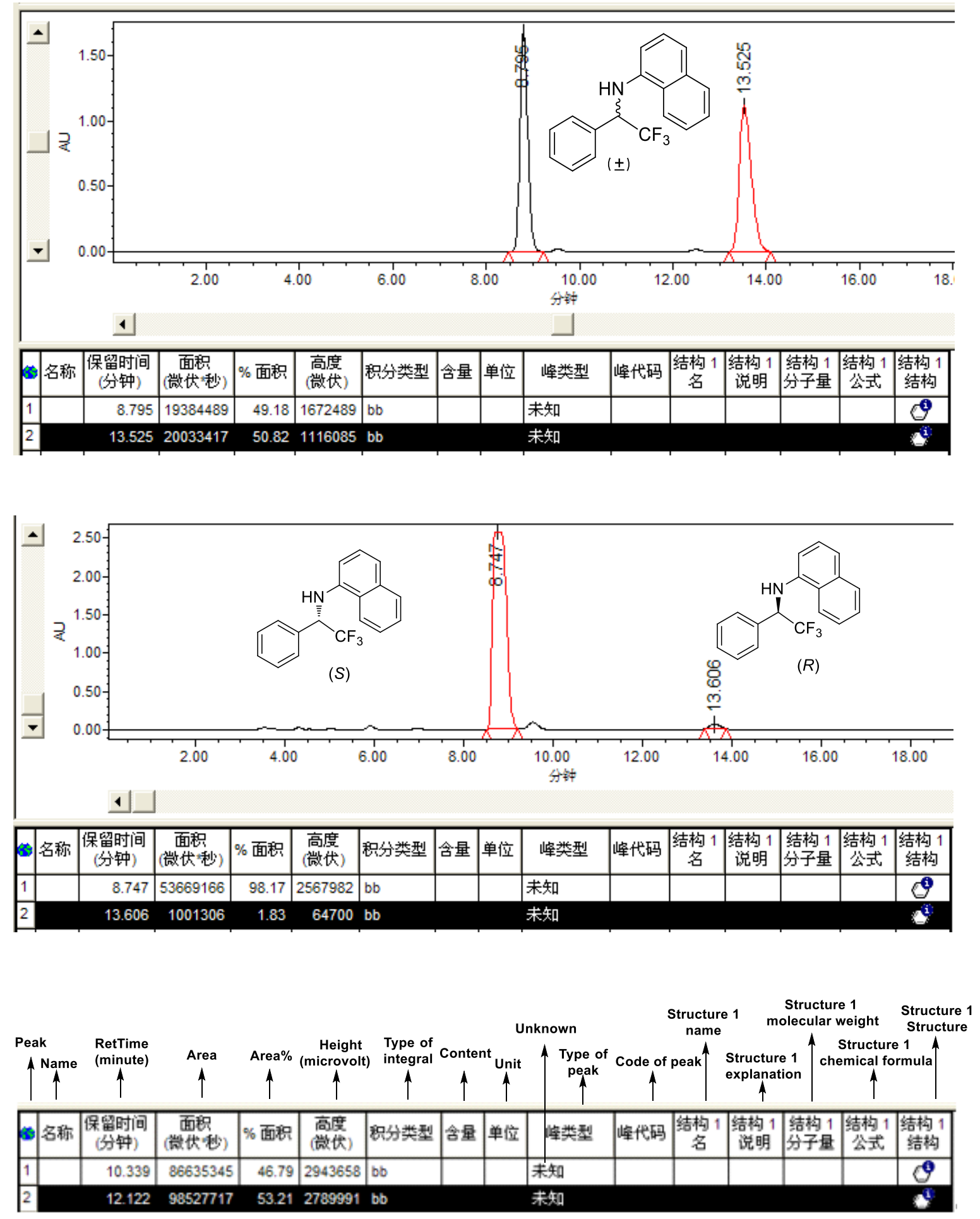

Figure S8. Asymmetric transfer hydrogenation of $\alpha$-trifluoromethylimines. [The products of ATH were analyzed by a HPLC with a UV-Vis detector using a Daicel OD-H or OB-H or

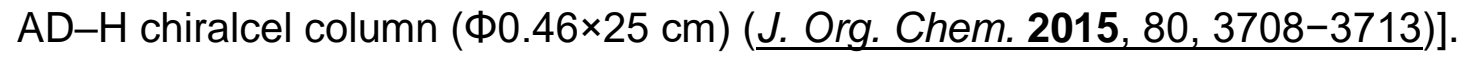


Table S1. Reusability of catalyst 3 using 4-methoxy-N-(2,2,2-trifluoro-1-(4fluorophenyl)ethylidene)aniline as a substrate. (Reaction conditions: catalyst 3 (195.60 mg, $40.0 \mu \mathrm{mol}$ of $\mathrm{Ru}$ based on ICP analysis), 4-methoxy-N-(2,2,2-trifluoro-1-(4fluorophenyl)ethylidene)aniline $(2.0 \mathrm{mmol}), 10.0 \mathrm{mmol}$ of HCOONa in $20.0 \mathrm{~mL}$ of water, and reaction time (16 h). The ee values were determined by chiral HPLC analysis.)

\begin{tabular}{lllllllll}
\hline Entry & $\mathbf{1}$ & $\mathbf{2}$ & $\mathbf{3}$ & $\mathbf{4}$ & $\mathbf{5}$ & $\mathbf{6}$ & $\mathbf{7}$ & $\mathbf{8}$ \\
\hline $\begin{array}{l}\text { Conversion } \\
\text { [\%] }\end{array}$ & 99 & 99 & 99 & 99 & 99 & 98 & 95 & 86 \\
ee [\%] & 96 & 96 & 96 & 96 & 95 & 95 & 96 & 91 \\
\hline
\end{tabular}

Recycling experiment part: Recycle 2.

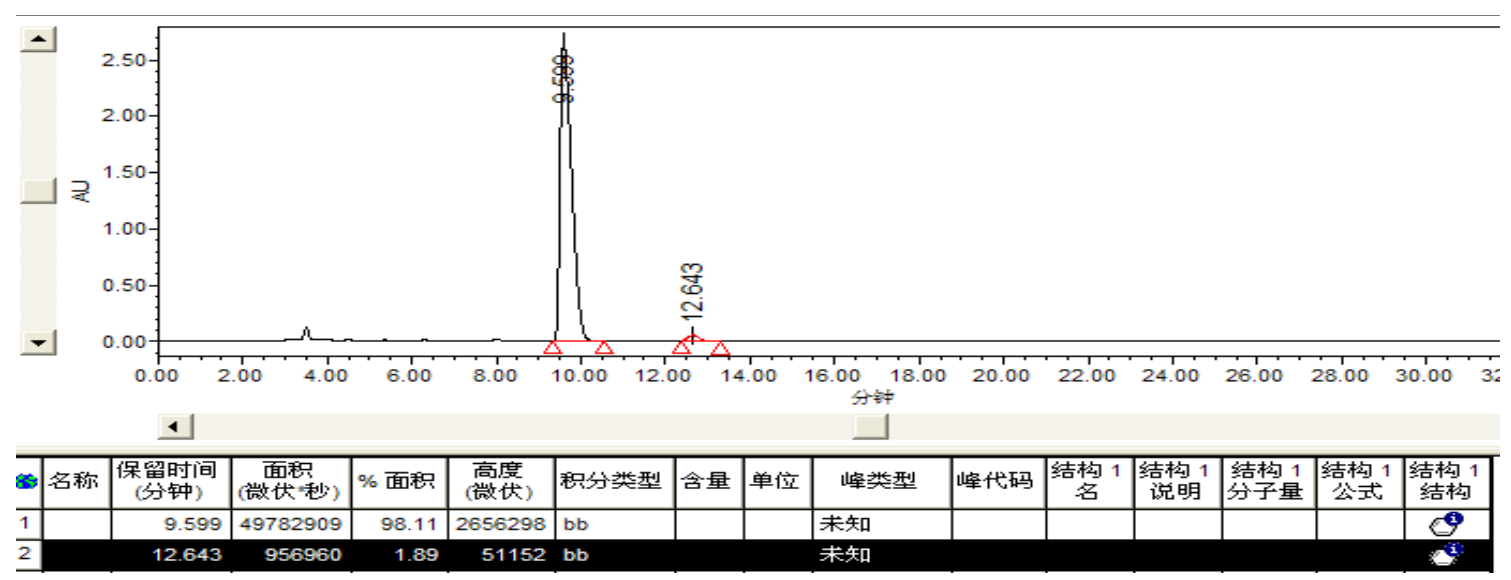

Recycle 3
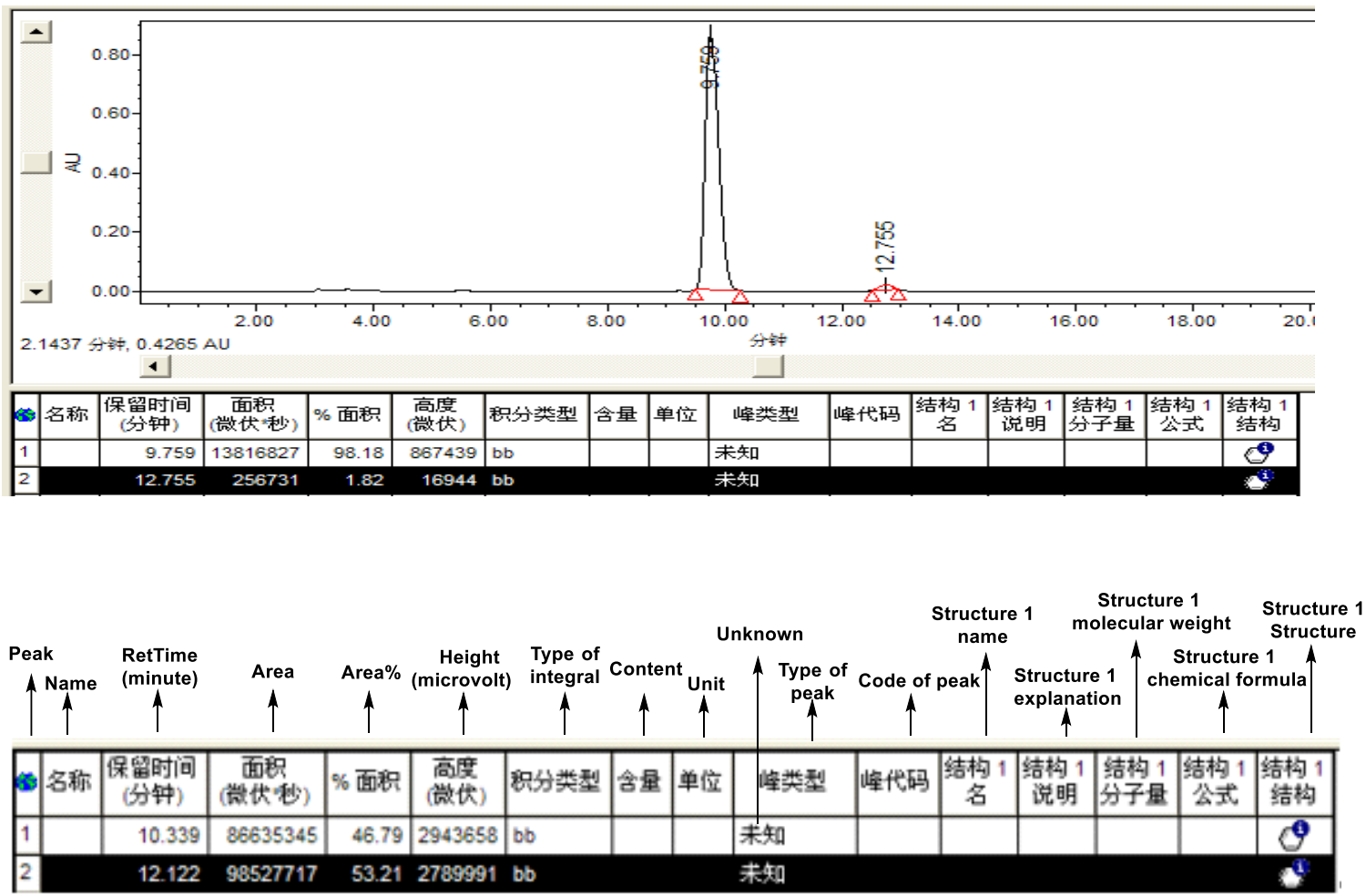


\section{Recycle 4}

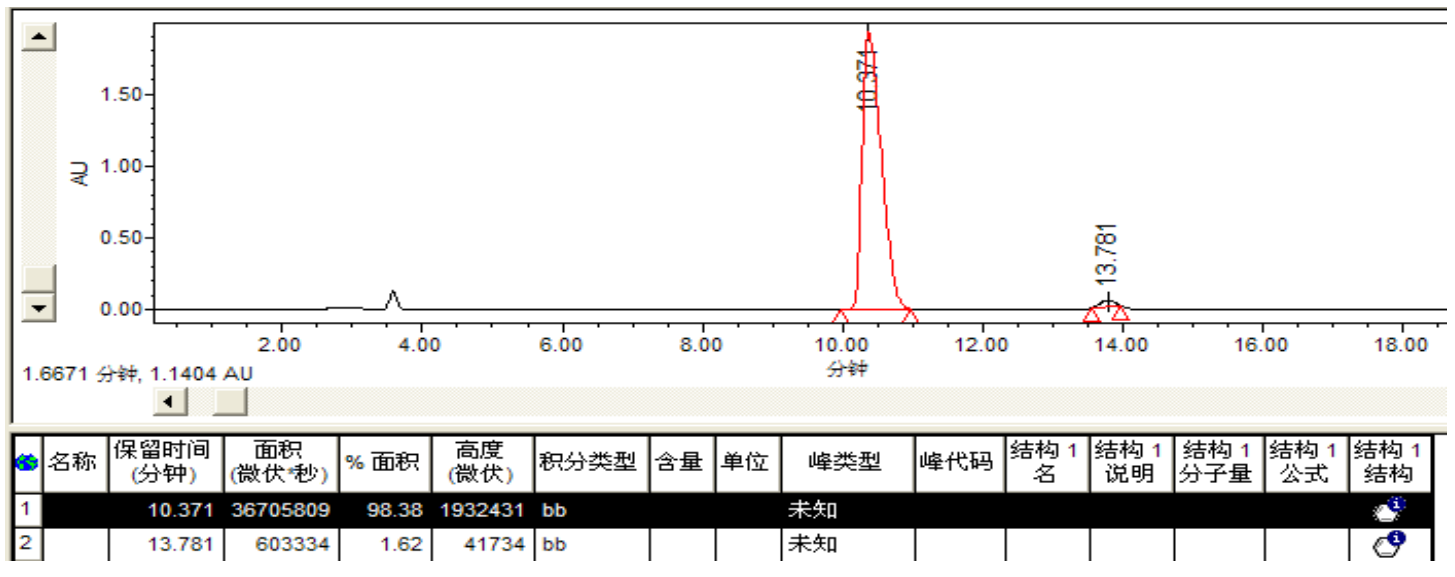

\section{Recycle 5}

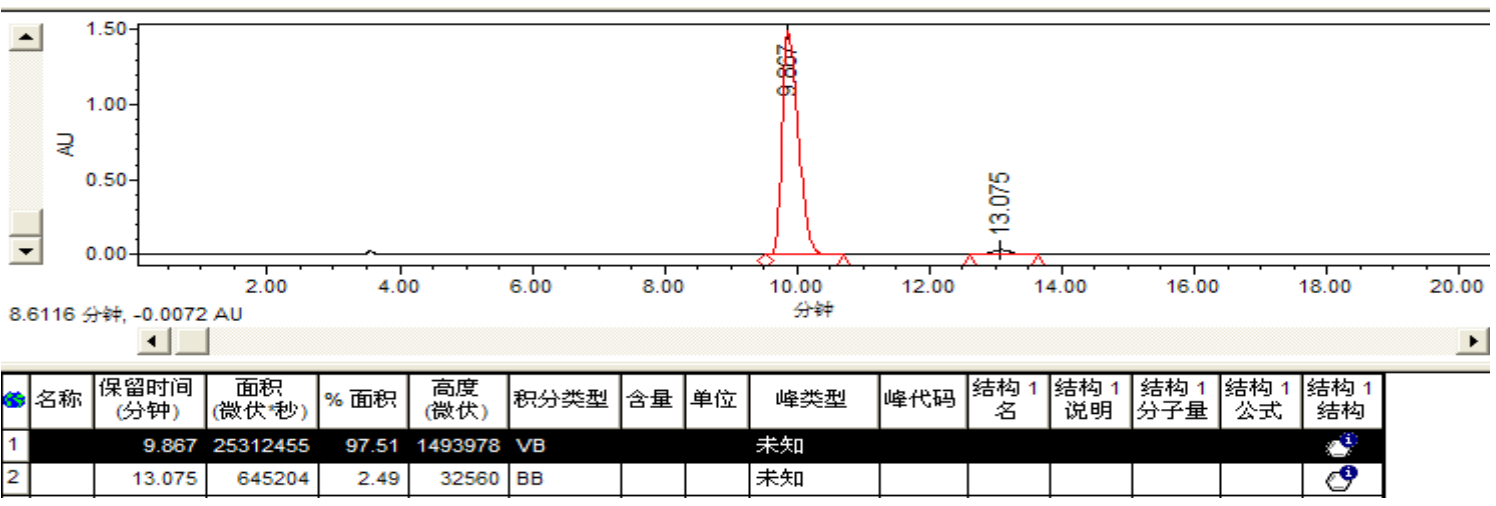

\section{Recycle 6}

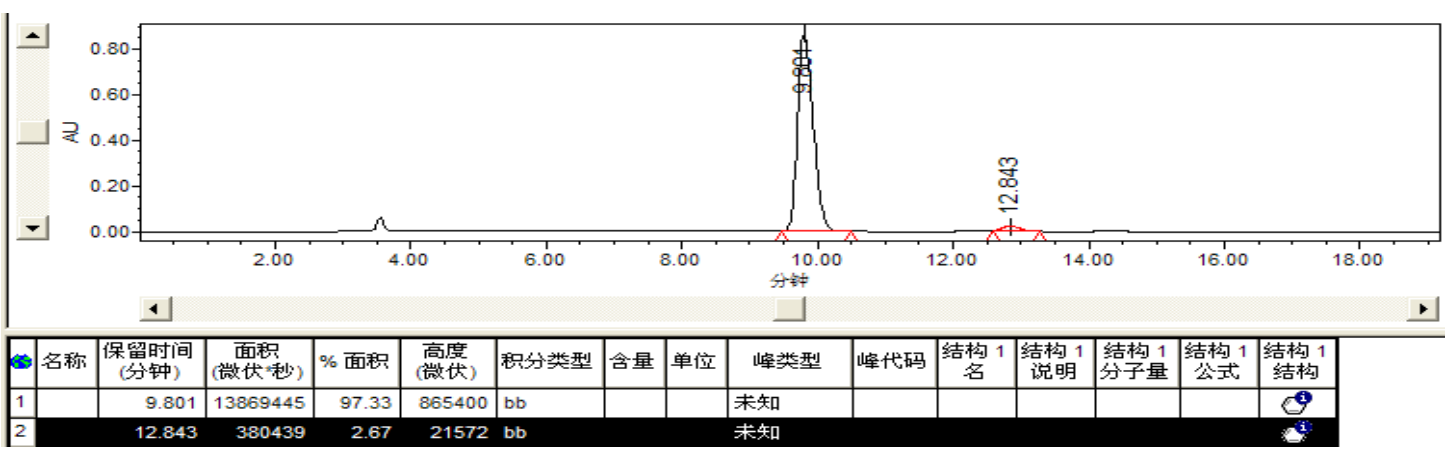

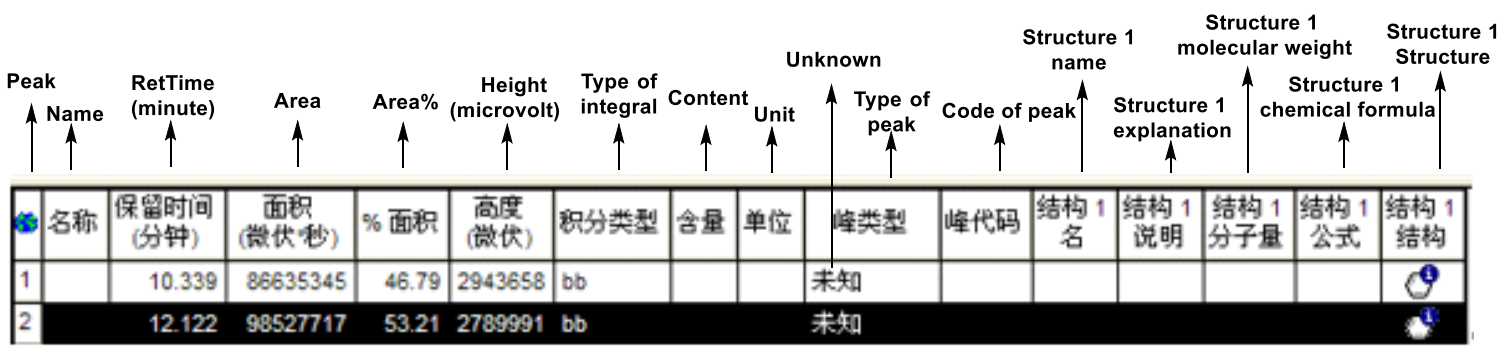


Recycle 7

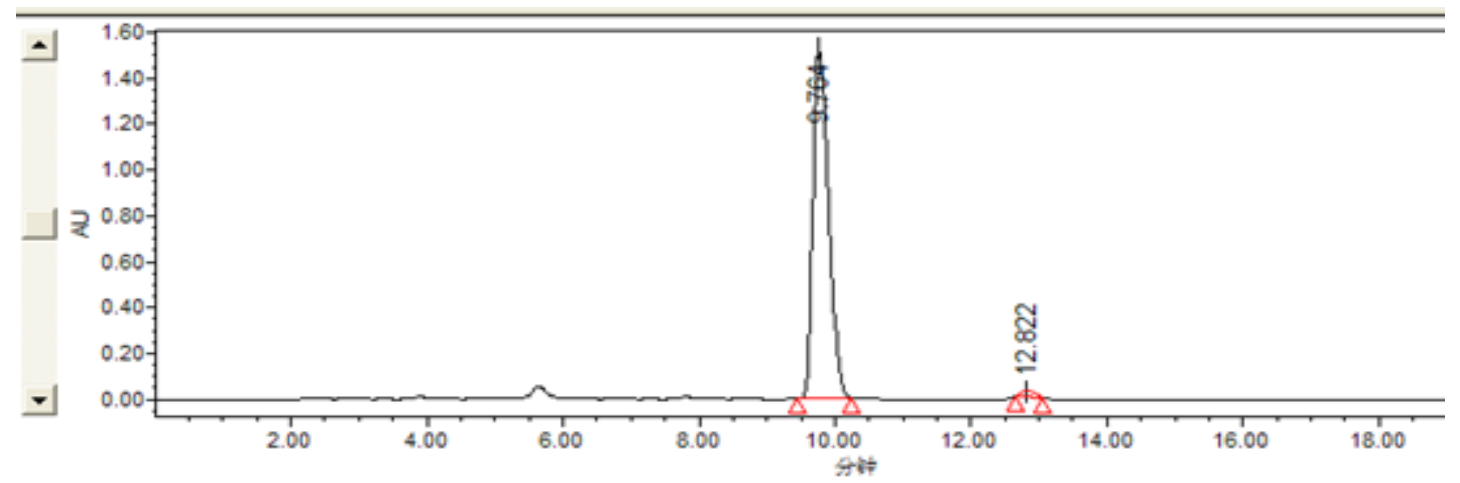

\begin{tabular}{|c|c|c|c|c|c|c|c|c|c|c|c|c|c|c|c|}
\hline 6 & 名称 & $\begin{array}{c}\text { 保留的间 } \\
\text { (分钟) }\end{array}$ & 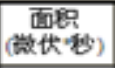 & \% 面积 & $\begin{array}{l}\text { 高曼 } \\
\text { (敞状) }\end{array}$ & 积分兴型 & 숭료 & 車位 & 峰尖型 & 峰代酖 & $\begin{array}{c}\text { 结詾 } 1 \\
\text { 名 }\end{array}$ & \begin{tabular}{|c|} 
结构 1 \\
说明
\end{tabular} & \begin{tabular}{|l|} 
结私 1 \\
分子量
\end{tabular} & \begin{tabular}{|l|} 
结构 1 \\
公式
\end{tabular} & \begin{tabular}{|l} 
结私 1 \\
结构
\end{tabular} \\
\hline 1 & & 9.764 & 24129719 & 98.33 & 1529150 & bo & & & 未知 & & & & & & 9 \\
\hline 2 & & 12.822 & 408700 & 1.67 & 29937 & bb & & & 未知 & & & & & & a) \\
\hline
\end{tabular}

\begin{tabular}{|c|c|c|c|c|c|c|c|c|c|c|c|c|c|c|c|c|}
\hline \multirow{2}{*}{\multicolumn{2}{|c|}{ Peak }} & \multirow[b]{2}{*}{$\begin{array}{l}\text { RetTime } \\
\text { (minute) }\end{array}$} & \multirow[b]{2}{*}{$\stackrel{\text { Area }}{\uparrow}$} & \multirow[b]{2}{*}{$\stackrel{\uparrow}{\text { Area } \%}$} & \multirow[b]{2}{*}{$\begin{array}{c}\text { Height } \\
\text { (microvolt) }\end{array}$} & \multirow[b]{2}{*}{$\begin{array}{c}\text { Type of } \\
\text { integral }\end{array}$} & \multicolumn{3}{|c|}{ Unknown } & \multicolumn{3}{|c|}{$\begin{array}{l}\text { Structure } 1 \\
\text { name }\end{array}$} & \multicolumn{3}{|c|}{$\begin{array}{c}\text { Structure } 1 \\
\text { molecular weight }\end{array}$} & $\begin{array}{l}\text { Structure } \\
\text { Structur } \\
1\end{array}$ \\
\hline & & & & & & & Content & $\stackrel{\text { Unit }}{\uparrow}$ & $\begin{array}{c}\text { Type of } \\
\text { peak }\end{array}$ & Code of $p$ & peak & $\begin{array}{l}\text { Structur } \\
\text { explanat }\end{array}$ & $\begin{array}{l}e 1 \\
\text { tion }\end{array}$ & chen & Structure & ermula \\
\hline 3 & 名称 & $\begin{array}{c}\text { 保留时间 } \\
\text { (分钟) }\end{array}$ & $\begin{array}{c}\text { 面积 } \\
\text { (阽仗批) }\end{array}$ & \% 面积 & $\begin{array}{l}\text { 高度 } \\
\text { (微湖) }\end{array}$ & 积分类型 & 암量 & 单位 & 峰类型 & 㤾代码 & $\begin{array}{c}\text { 结构 } 1 \\
\text { 名 }\end{array}$ & $\begin{array}{l}\text { 结构 } 1 \\
\text { 说明 }\end{array}$ & 结柆 & $\begin{array}{l}1 \\
1\end{array}$ & \begin{tabular}{|l|} 
结构 1 \\
公式
\end{tabular} & $\begin{array}{c}\text { 结构 } 1 \\
\text { 结构 }\end{array}$ \\
\hline 1 & & 10.339 & 86635345 & 46.79 & 2943658 & $b b$ & & & 未知 & & & & & & & 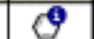 \\
\hline 2 & & 12.122 & 98527717 & 53.21 & 2789991 & bb & & & 米知 & & & & & & & d \\
\hline
\end{tabular}

Figure S9. Reusability of catalyst 3 using 4-methoxy-N-(2,2,2-trifluoro-1-(4fluorophenyl)ethylidene)aniline as a substrate. 
(S)-3-(2-nitro-1-phenylethyl)pentane-2,4-dione (Literature: Chem. Eur. J. 2010, 16, 6748). (HPLC: Chiracel AD-H, detected at $215 \mathrm{~nm}$, eluent: $\mathrm{n}$-hexane/2-propanol $=90 / 10$, flow rate $=1.0 \mathrm{~mL} / \mathrm{min}, 25^{\circ} \mathrm{C}, \mathrm{t}_{1}=8.9 \mathrm{~min}(\mathrm{maj}), \mathrm{t}_{2}=11.6 \mathrm{~min}$ ).
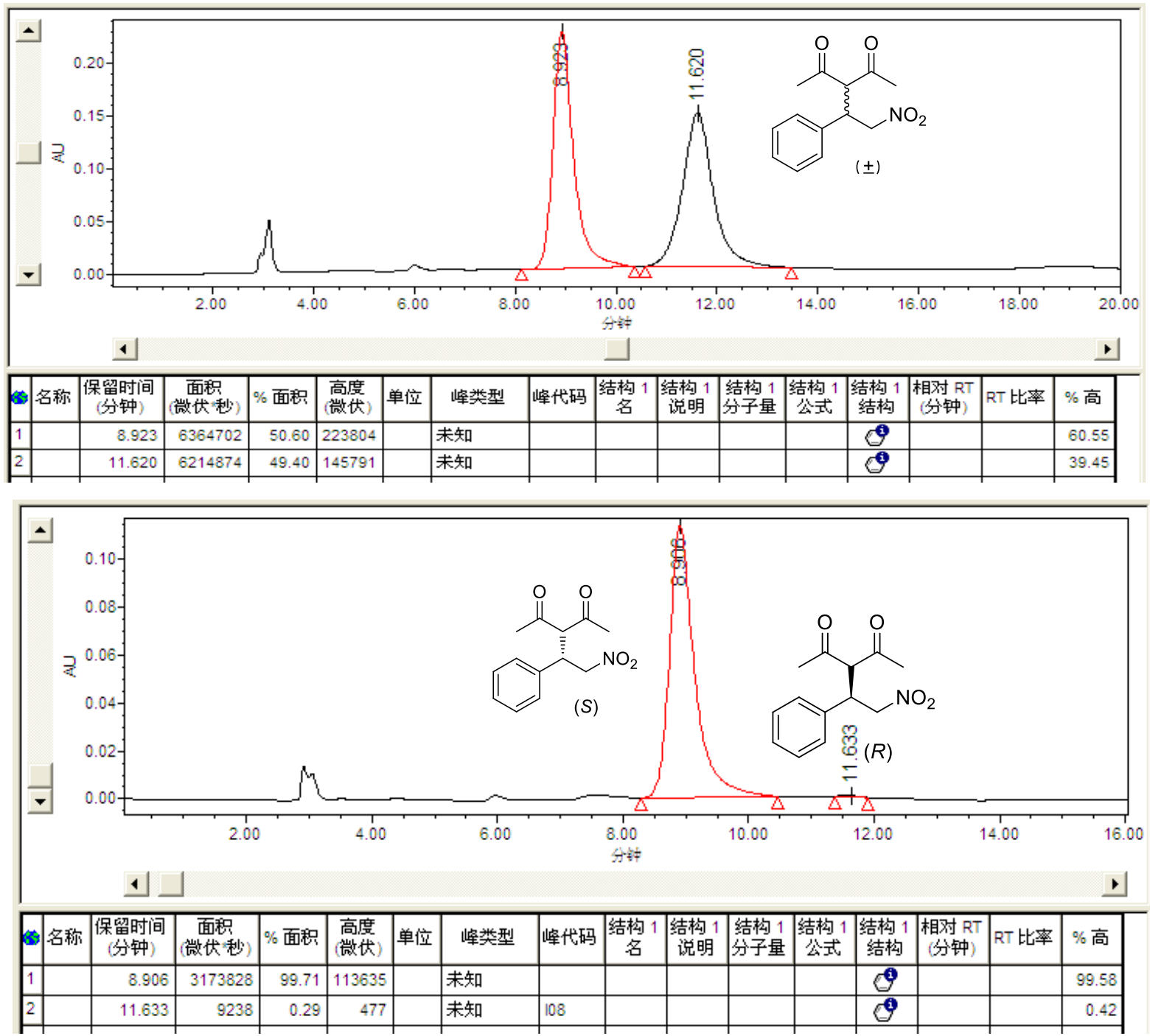

\begin{tabular}{|c|c|c|c|c|c|c|c|c|c|c|c|c|c|c|c|c|}
\hline \multirow{2}{*}{\multicolumn{2}{|c|}{$\uparrow^{\text {Peak }} \uparrow^{\text {Name }}$}} & \multirow{2}{*}{$\begin{array}{c}\text { RetTime } \\
\text { (minute) } \\
\uparrow_{(\mathrm{mi}}\end{array}$} & \multirow{2}{*}{\multicolumn{2}{|c|}{$\begin{array}{c}\text { Area } \\
\text { crovolt/second) }\end{array} \uparrow$}} & \multirow{2}{*}{\multicolumn{3}{|c|}{ Unit $\uparrow_{\uparrow}^{\text {Unknown of }}$}} & \multicolumn{5}{|c|}{$\begin{array}{cc}\begin{array}{c}\text { Structure } 1 \\
\text { name }\end{array} & \text { Structure } 1 \\
\text { molecular weight }\end{array}$} & \multicolumn{2}{|c|}{$\begin{array}{l}\text { Structure } 1 \\
\text { Structure }\end{array}$} & \multicolumn{2}{|r|}{ Height $\%$} \\
\hline & & & & & & & & $\begin{array}{c}\text { Code of } \\
\text { peak }\end{array}$ & & $\begin{array}{l}\text { tructure } 1 \\
\text { xplanation }\end{array}$ & $1 \uparrow$ che & $\begin{array}{c}\text { Structure } \\
\text { mical fo } \\
\uparrow\end{array}$ & $\begin{array}{l}\text { e } 1 \uparrow \\
\text { ormula }\end{array}$ & $\begin{array}{l}\text { RetTime } \\
\text { (minute) }\end{array}$ & $\begin{array}{l}\text { Ratio of } \\
\text { RetTime }\end{array}$ & חetgitio \\
\hline 3 & 8 名繁 & 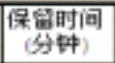 & 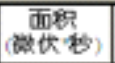 & $\%$ 西的 & 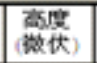 & 事位 & 㧹来型 & 俥:代的 & 结构 1 & \begin{tabular}{|c|} 
\\
结极 1 \\
说明
\end{tabular} & $\begin{array}{l}\text { 结鸱 } 1 \\
\text { 分子量 }\end{array}$ & \begin{tabular}{|c|} 
结构 1 \\
公式
\end{tabular} & $\begin{array}{l}\text { 結被 } 1 \\
\text { 结枮 }\end{array}$ & 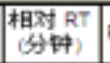 & RT 比雪 & $\%$ 高 \\
\hline 1 & & 8.906 & 3173828 & 99.71 & 113635 & & 乘如 & & & & & & 9 & & & 99.58 \\
\hline 2 & & 11.633 & 9238 & 0.29 & 477 & & 来知 & 108 & & & & & 6 & & & 0.42 \\
\hline
\end{tabular}


(S)-3-(1-(4-fluorophenyl)-2-nitroethyl)pentane-2,4-dione (Literature: Green Chem. 2012. 14, 893)._(HPLC: Chiracel OD-H, detected at $215 \mathrm{~nm}$, eluent: n-hexane/2-propanol $=85 / 15$, flow rate $\left.=1.0 \mathrm{~mL} / \mathrm{min}, 25^{\circ} \mathrm{C}, \mathrm{t}_{1}=14.2 \mathrm{~min}(\mathrm{maj}), \mathrm{t}_{2}=15.8 \mathrm{~min}\right)$.
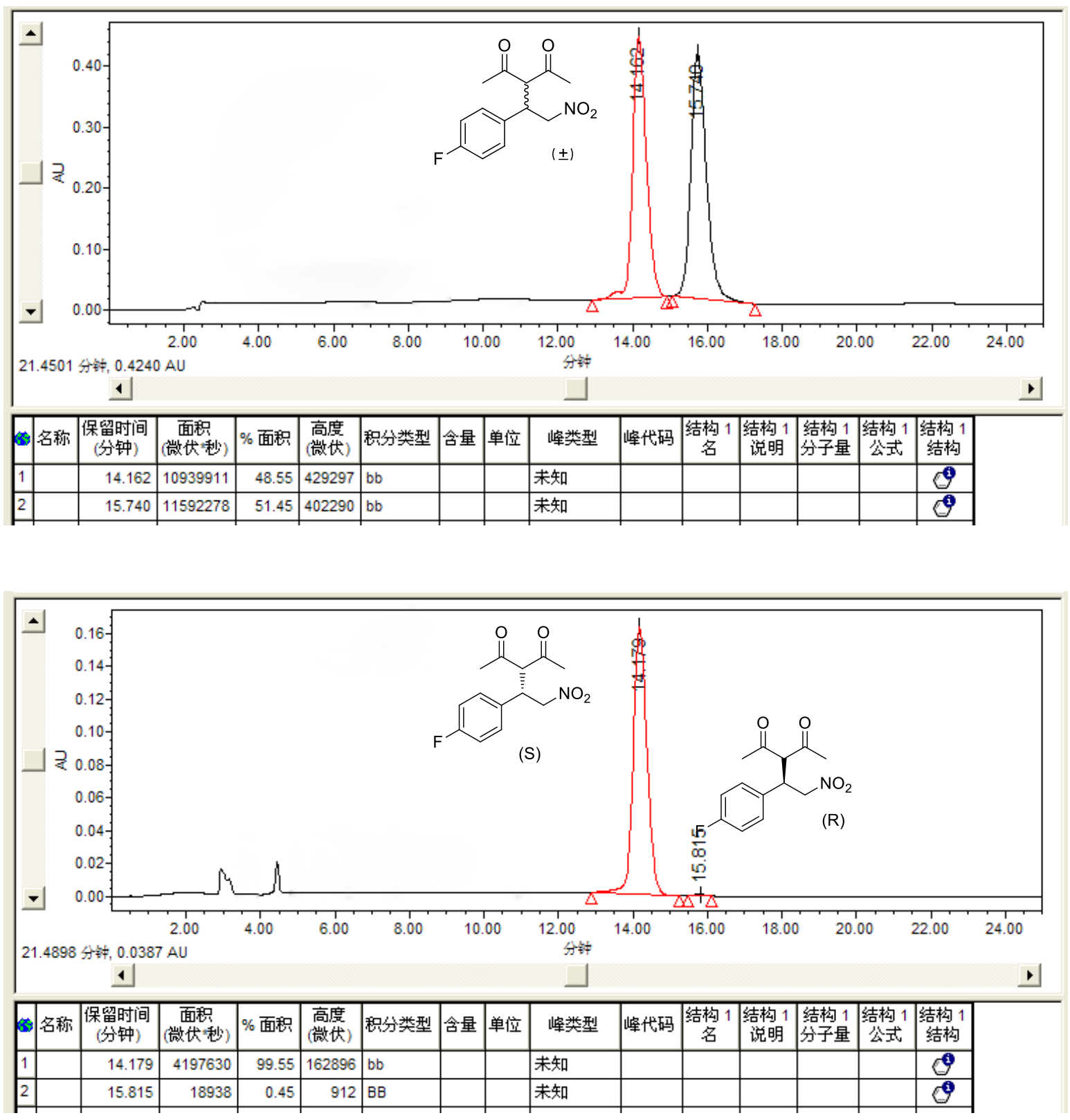

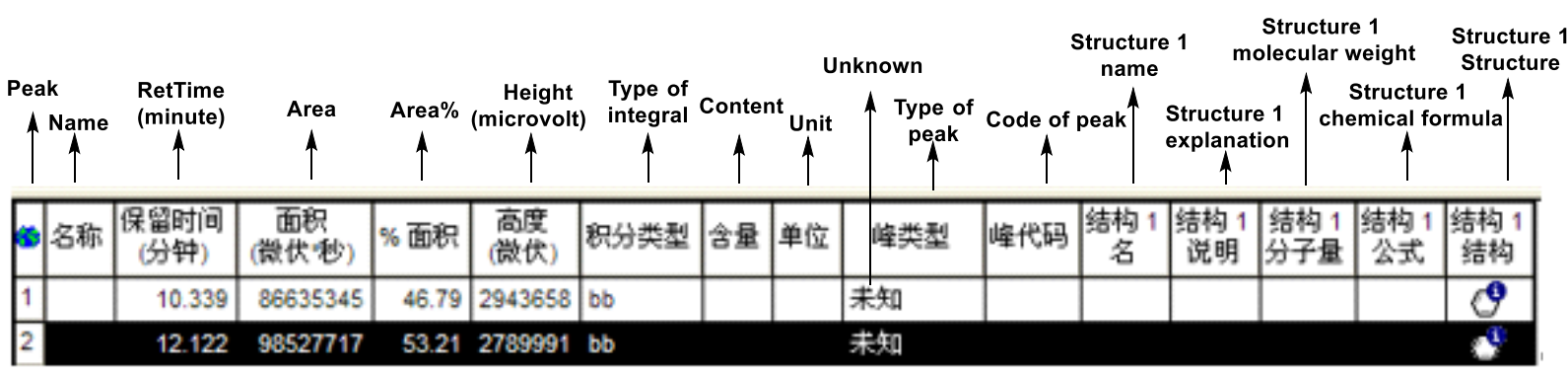


(S)-3-(1-(4-chlorophenyl)-2-nitroethyl)pentane-2,4-dione (Literature: Green Chem. 2012, 14, 893). (HPLC: Chiracel AS-H, detected at $215 \mathrm{~nm}$, eluent: $\mathrm{n}$-hexane/2-propanol $=85 / 15$, flow rate $=1.0 \mathrm{~mL} / \mathrm{min}, 25^{\circ} \mathrm{C}, \mathrm{t}_{1}=13.0 \mathrm{~min}$ (maj), $\mathrm{t}_{2}=15.4 \mathrm{~min}$ ).
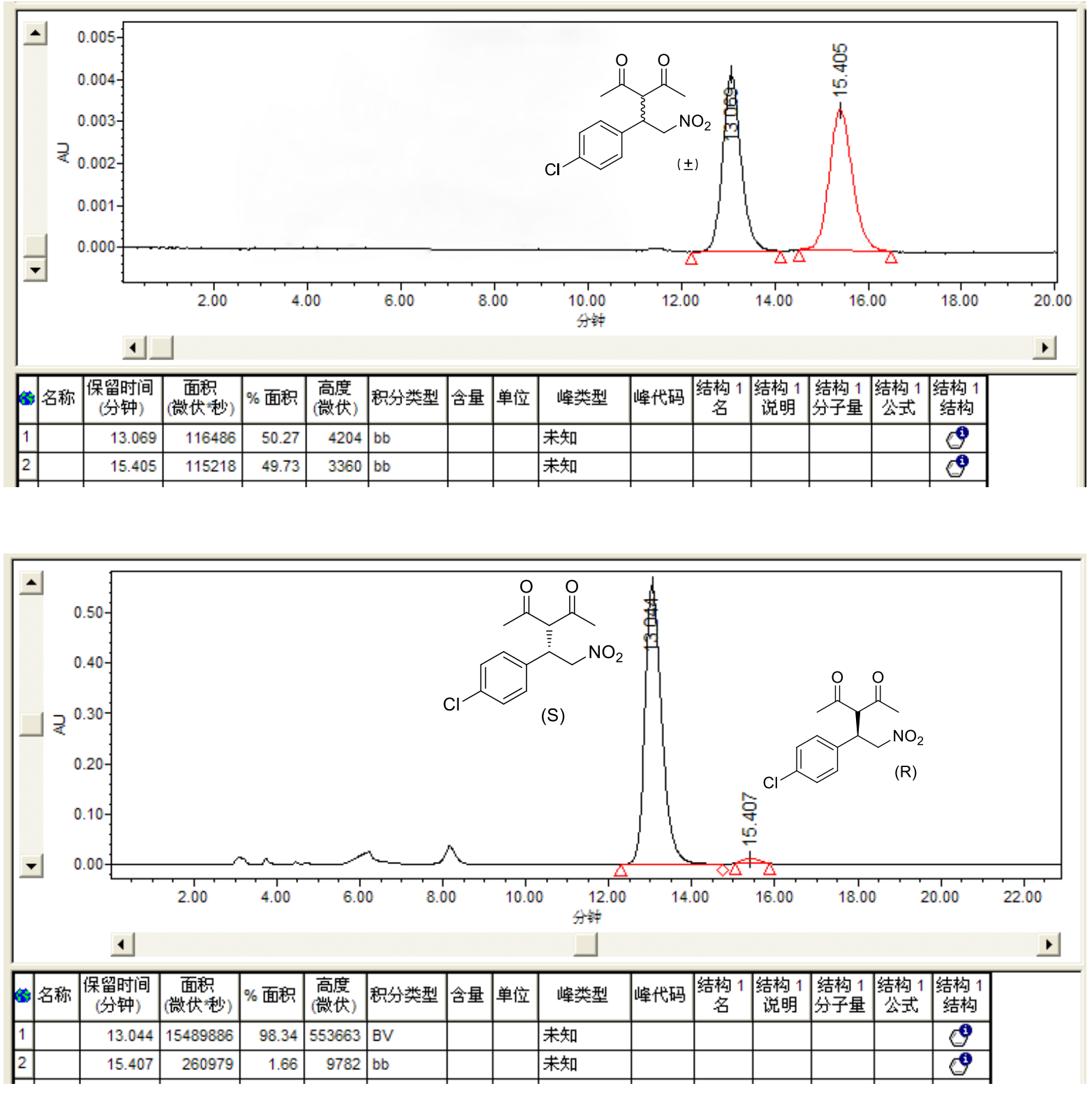

\begin{tabular}{|c|c|c|c|c|c|c|c|c|c|c|c|c|c|c|}
\hline \multirow[b]{2}{*}{ Peak } & \multirow[b]{2}{*}{$\begin{array}{c}\text { RetTime } \\
\text { (minute) }\end{array}$} & \multirow[b]{2}{*}{$\stackrel{\text { Area }}{\uparrow}$} & \multirow[b]{2}{*}{ Area\% } & \multirow[b]{2}{*}{$\begin{array}{c}\text { Height } \\
\text { (microvolt) }\end{array}$} & \multirow[b]{2}{*}{$\begin{array}{c}\text { Type of } \\
\text { integral }\end{array}$} & \multicolumn{3}{|c|}{ Unknown } & \multicolumn{2}{|c|}{$\begin{array}{c}\text { Structure } 1 \\
\text { name }\end{array}$} & \multicolumn{3}{|c|}{$\begin{array}{c}\text { Structure } 1 \\
\text { molecular weight }\end{array}$} & $\begin{array}{l}\text { Structure } \\
\text { Structur }\end{array}$ \\
\hline & & & & & & Conte & nt Unit & Type of & Code of & f peak $\uparrow$ & $\begin{array}{l}\text { Structure } \\
\text { explanatic } \\
\uparrow\end{array}$ & $\begin{array}{l}\text { e } 1 \\
\text { tion }\end{array}$ & $\begin{array}{c}\text { Structure } \\
\text { hemical for }\end{array}$ & $\begin{array}{l}\text { re } 1 \\
\text { ormula }\end{array}$ \\
\hline 3 名称 & $\begin{array}{l}\text { 保留时间 } \\
\text { (分钟) }\end{array}$ & $\begin{array}{l}\text { 面积 } \\
\text { (敞代制) }\end{array}$ & \% 面积 & $\begin{array}{l}\text { 高度 } \\
\text { (溦伏) }\end{array}$ & 积分类型 & 㕣量 & 半位 & 峰尖型 & 峰代码 & $\begin{array}{l}\text { 结构 } 1 \\
\text { 名 }\end{array}$ & $\mid$\begin{tabular}{|c|}
$\mid$ 结构 1 \\
说明
\end{tabular} & $\begin{array}{l}\text { 结构 } 1 \\
\text { 分子量 }\end{array}$ & \begin{tabular}{|l|l|}
1 & 结构 1 \\
& 公式
\end{tabular} & \begin{tabular}{|l|} 
\\
$\mid \begin{array}{l}\text { 结构 } 1 \\
\text { 结构 }\end{array}$
\end{tabular} \\
\hline 1 & 10.339 & 86635345 & 46.79 & 2943658 & bo & & & 来知 & & & & & & 9 \\
\hline 2 & 12.122 & 98527717 & 53.21 & 2789991 & $b b$ & & & 末知 & & & & & & s \\
\hline
\end{tabular}


(S)-3-(1-(3-chlorophenyl)-2-nitroethyl)pentane-2,4-dione (Literature: Green Chem. 2012, 14, 893). (HPLC: Chiracel AS-H, detected at $215 \mathrm{~nm}$, eluent: $\mathrm{n}$-hexane/2-propanol $=85 / 15$, flow rate $\left.=1.0 \mathrm{~mL} / \mathrm{min}, 25^{\circ} \mathrm{C}, \mathrm{t}_{1}=13.6 \mathrm{~min}(\mathrm{maj}), \mathrm{t}_{2}=23.7 \mathrm{~min}\right)$.
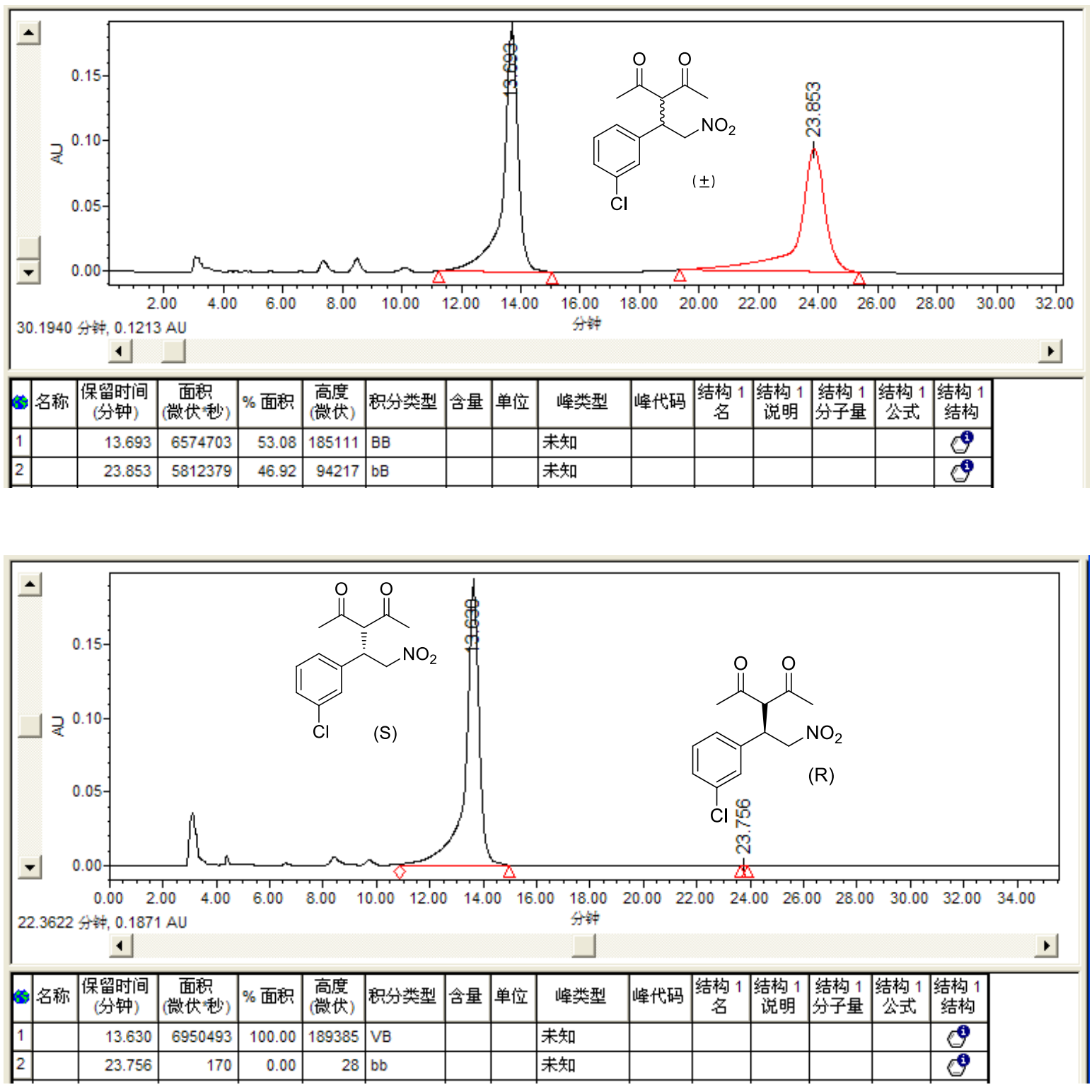

\begin{tabular}{|c|c|c|c|c|c|c|c|c|c|c|c|c|c|c|}
\hline \multirow[b]{2}{*}{$\stackrel{\text { Peak }}{\text { Name }}$} & \multirow[b]{2}{*}{$\begin{array}{c}\text { RetTime } \\
\text { (minute) }\end{array}$} & \multirow[b]{2}{*}{$\stackrel{\text { Area }}{\uparrow}$} & \multirow[b]{2}{*}{$\stackrel{\text { Area } \%}{\uparrow}$} & \multirow[b]{2}{*}{$\begin{array}{c}\text { Height } \\
\text { (microvolt) }\end{array}$} & \multirow[b]{2}{*}{$\begin{array}{c}\begin{array}{c}\text { Type of } \\
\text { integral }\end{array} \\
\end{array}$} & \multicolumn{3}{|c|}{ Unknown } & \multicolumn{2}{|c|}{$\begin{array}{c}\text { Structure } 1 \\
\text { name }\end{array}$} & \multicolumn{3}{|c|}{$\begin{array}{c}\text { Structure } 1 \\
\text { molecular weight }\end{array}$} & \\
\hline & & & & & & Conten & ${ }^{\text {nt }}$ Unit & $\underset{\substack{\text { Type of } \\
\text { peak }}}{\uparrow}$ & Code of & peak $\uparrow$ & $\begin{array}{l}\text { Structure } \\
\text { explanati }\end{array}$ & $\begin{array}{l}\text { re } 1 \\
\text { tion }\end{array} \uparrow \mathrm{ch}$ & $\begin{array}{l}\text { Structur } \\
\text { chemical fo }\end{array}$ & \\
\hline 3 名称 & $\begin{array}{l}\text { 保留时间 } \\
\text { (分钟) }\end{array}$ & $\begin{array}{c}\text { 面积 } \\
\text { (耀伏制) }\end{array}$ & \% 面积 & $\begin{array}{l}\text { 高度 } \\
\text { (溦伏) }\end{array}$ & 积分类型 & 合量 & 单位 & 峰带型 & 深代码 & \begin{tabular}{|l} 
结构 1 \\
名
\end{tabular} & \begin{tabular}{|c|} 
结构 1 \\
说明
\end{tabular} & $\begin{array}{l}\text { 结构 } 1 \\
\text { 分子量 }\end{array}$ & 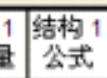 & \begin{tabular}{|l}
1 \\
$\mid \begin{array}{l}\text { 结构 } 1 \\
\text { 结构 }\end{array}$
\end{tabular} \\
\hline 1 & 10.339 & 86635345 & 46.79 & 2943658 & bo & & & 来知 & & & & & & (9) \\
\hline 2 & 12.122 & 98527717 & 53.21 & 2789991 & $b b$ & & & 未知 & & & & & & is \\
\hline
\end{tabular}


(S)-3-(1-(2-chlorophenyl)-2-nitroethyl)pentane-2,4-dione (Literature: Green Chem. 2012. 14, 893). (HPLC: Chiracel AD-H, detected at $215 \mathrm{~nm}$, eluent: $n$-hexane/2-propanol $=98 / 02$, flow rate $\left.=1.0 \mathrm{~mL} / \mathrm{min}, 25^{\circ} \mathrm{C}, \mathrm{t}_{1}=10.6 \mathrm{~min}(\mathrm{maj}), \mathrm{t}_{2}=11.9 \mathrm{~min}\right)$.
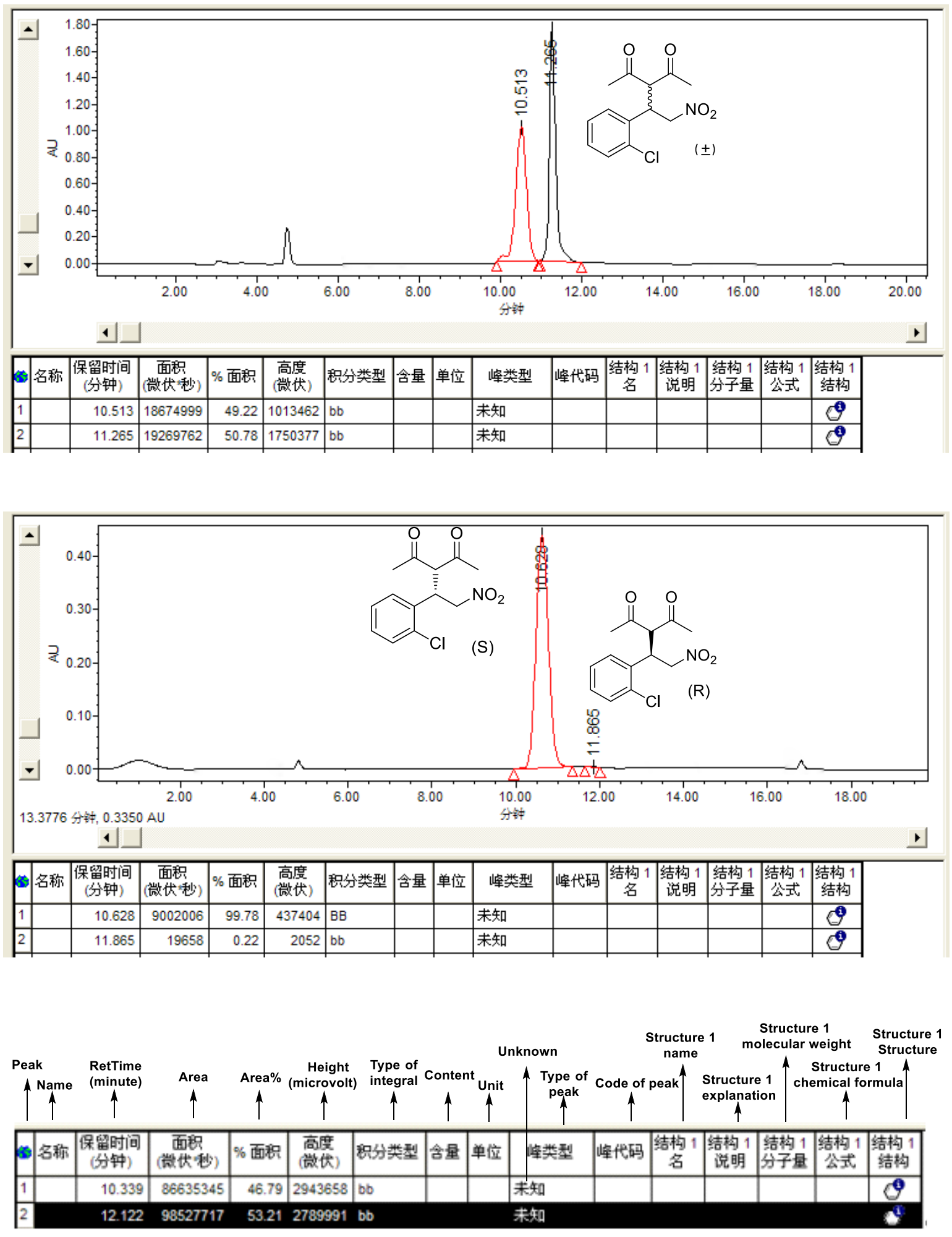
(S)-3-(1-(4-bromophenyl)-2-nitroethyl)pentane-2,4-dione (Literature: Green Chem. 2012. 14, 893). (HPLC: Chiracel OD-H, detected at $215 \mathrm{~nm}$, eluent: $\mathrm{n}$-hexane/2-propanol $=90 / 10$, flow rate $\left.=1.0 \mathrm{~mL} / \mathrm{min}, 25^{\circ} \mathrm{C}, \mathrm{t}_{1}=23.3 \mathrm{~min}(\mathrm{maj}), \mathrm{t}_{2}=24.9 \mathrm{~min}\right)$.
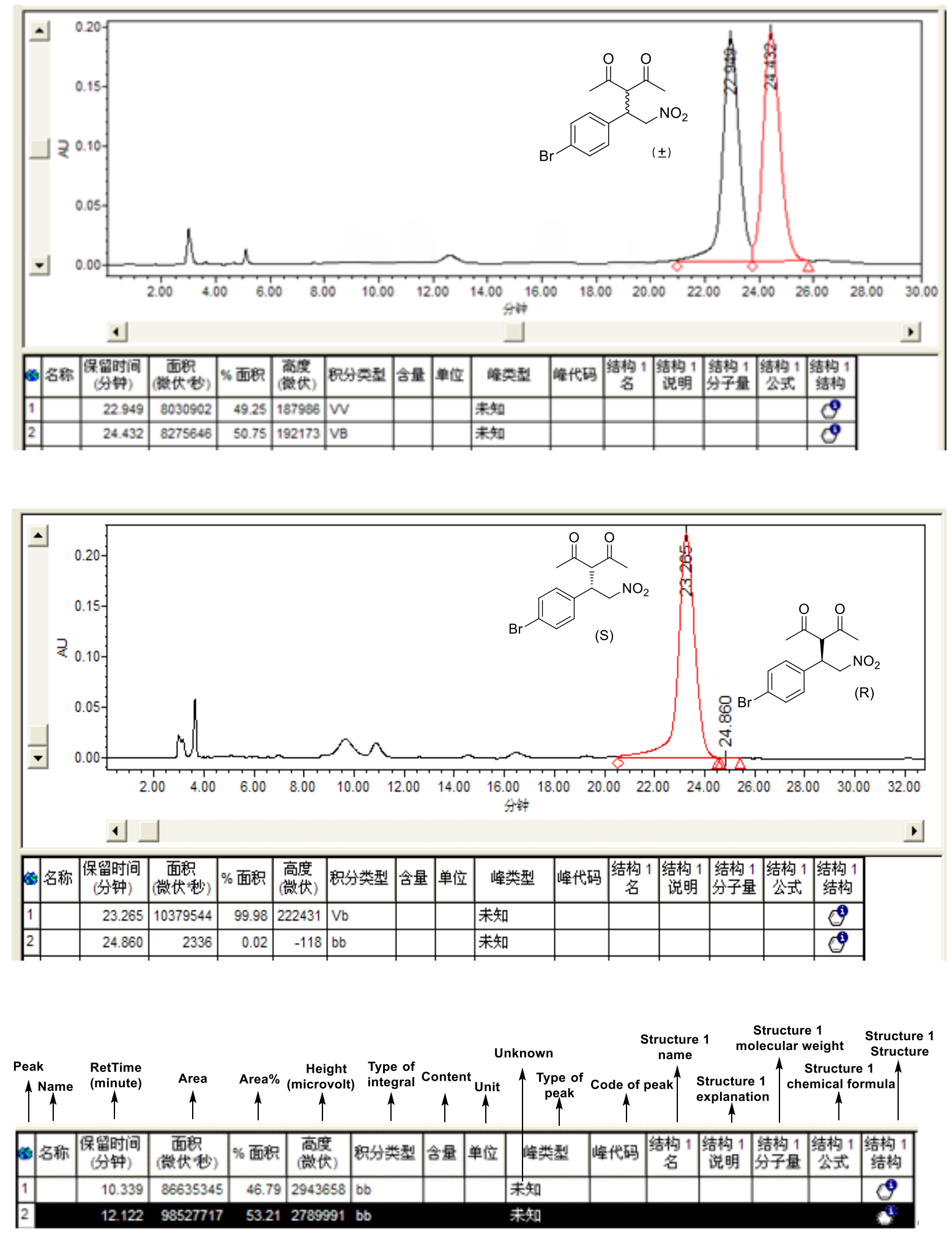
(S)-3-(2-nitro-1-(4-(trifluoromethyl)phenyl)ethyl)pentane-2,4-dione (Literature: Catal. Sci. Technol. 2014, 4, 1726). (HPLC: Chiracel AS-H, detected at $215 \mathrm{~nm}$, eluent: $\mathrm{n}$-hexane/2propanol $=95 / 05$, flow rate $=1.0 \mathrm{~mL} / \mathrm{min}, 25^{\circ} \mathrm{C}, \mathrm{t}_{1}=21.6 \mathrm{~min}(\mathrm{maj}), \mathrm{t}_{2}=36.2 \mathrm{~min}$ ).
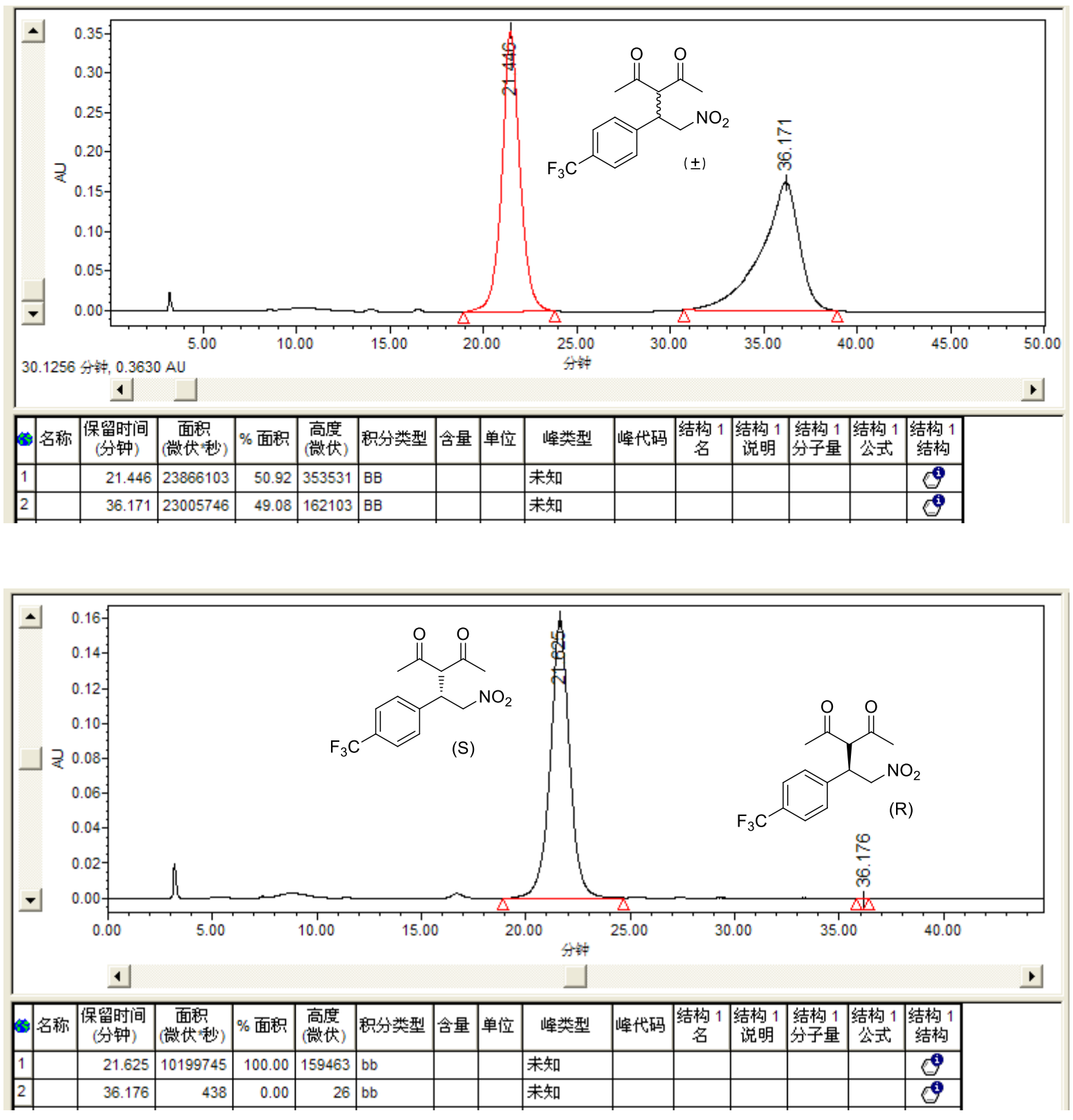

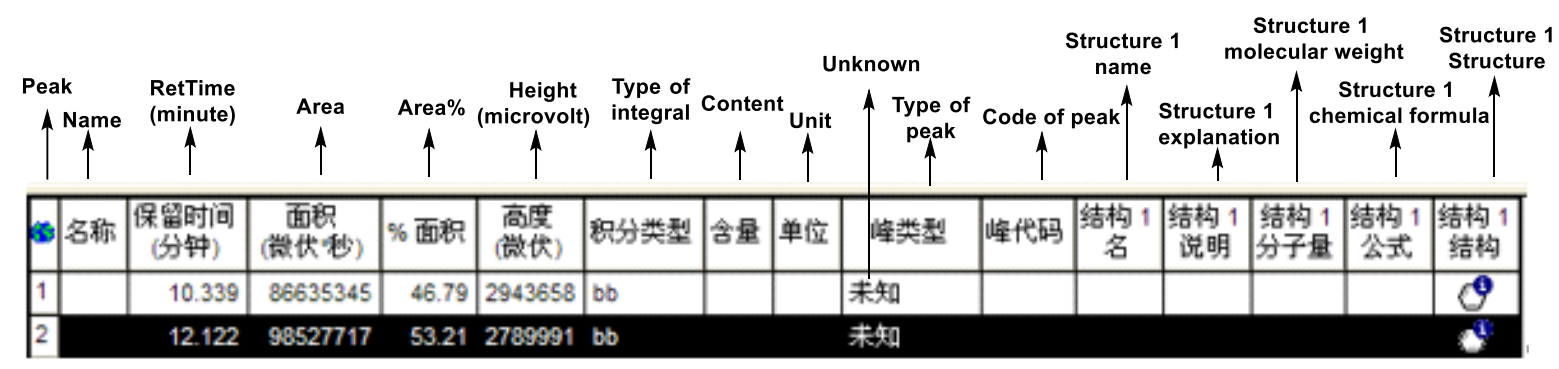


(S)-3-(1-(4-methylphenyl)-2-nitroethyl)pentane-2,4-dione (Literature: ACS Catal. 2014, 4, 2137). (HPLC: Chiracel AD-H, detected at $215 \mathrm{~nm}$, eluent: $n$-hexane/2-propanol $=90 / 10$, flow rate $=1.0 \mathrm{~mL} / \mathrm{min}, 25^{\circ} \mathrm{C}, \mathrm{t}_{1}=8.5 \mathrm{~min}(\mathrm{maj}), \mathrm{t}_{2}=13.2 \mathrm{~min}$ ).
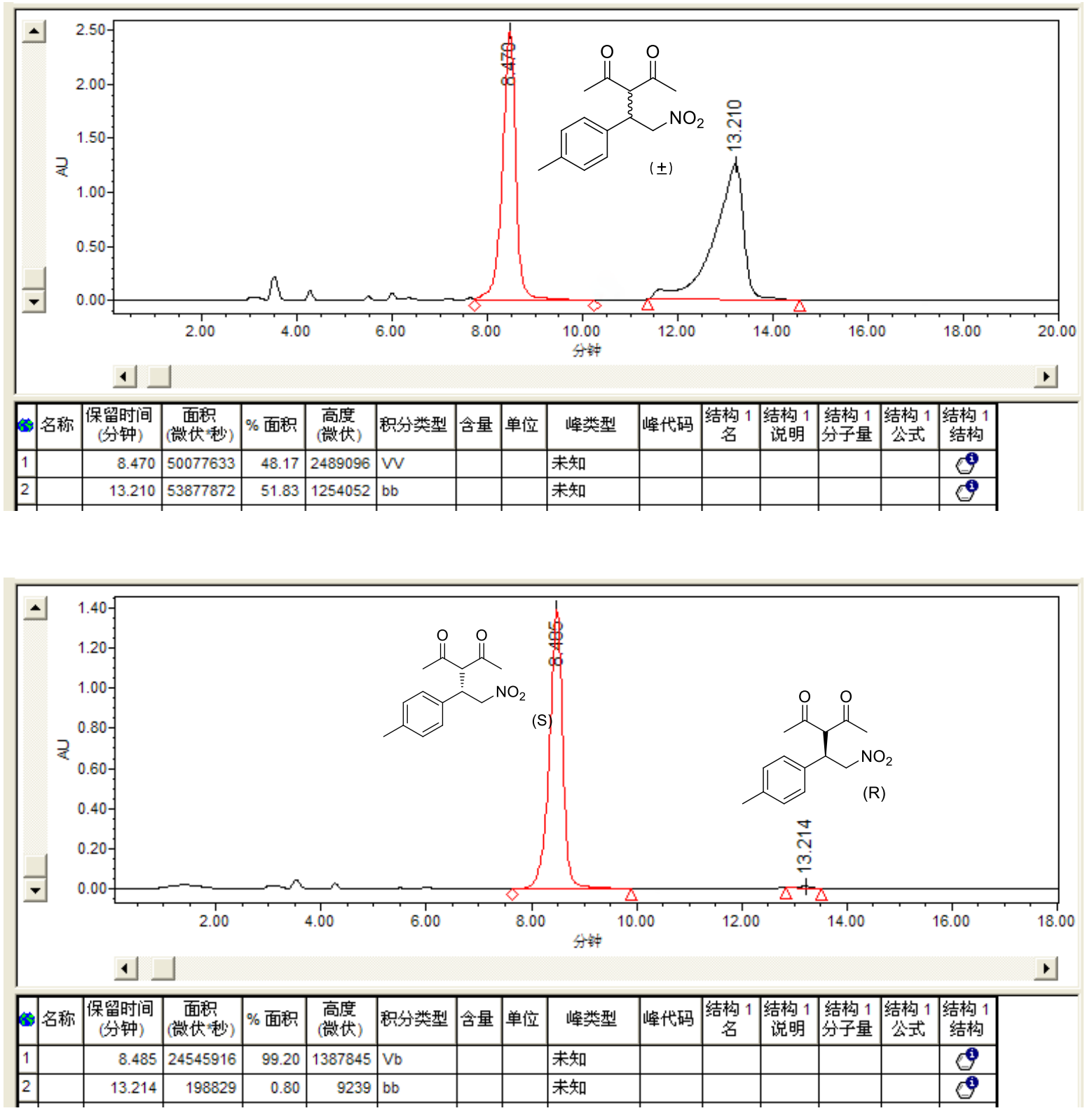

\begin{tabular}{|c|c|c|c|c|c|c|c|c|c|c|c|c|c|c|c|}
\hline \multirow{2}{*}{\multicolumn{2}{|c|}{ Peak }} & \multirow[b]{2}{*}{$\stackrel{\substack{\text { RetTime } \\
\text { (minute) }}}{\uparrow}$} & \multirow[b]{2}{*}{ Area } & \multirow[b]{2}{*}{ Area\% } & \multirow[b]{2}{*}{$\begin{array}{c}\begin{array}{c}\text { Height } \\
\text { (microvolt }\end{array} \\
\uparrow\end{array}$} & \multirow[b]{2}{*}{$\begin{array}{c}\text { Type of } \\
\text { integral }\end{array}$} & \multicolumn{3}{|c|}{ Unknown } & \multicolumn{3}{|c|}{$\begin{array}{c}\text { Structure } 1 \\
\text { name }\end{array}$} & \multicolumn{2}{|c|}{$\begin{array}{l}\text { Structure } 1 \\
\text { molecular weight }\end{array}$} & $\begin{array}{l}\text { Structure } \\
\text { Structure }\end{array}$ \\
\hline & & & & & & & Conten & ${ }_{\uparrow}^{\text {Unit }}$ & $\underset{\substack{\text { Type of } \\
\text { peak }}}{\uparrow}$ & Code of & peak $\uparrow$ & $\begin{array}{l}\text { Structur } \\
\text { explanat }\end{array}$ & $\begin{array}{l}\text { re } 1 \\
\text { tion }\end{array} \uparrow \mathrm{ch}$ & Structur & re 1 ormula \\
\hline 3 & 名称 & $\begin{array}{l}\text { 保留时间 } \\
\text { (分钟) }\end{array}$ & $\begin{array}{c}\text { 面积 } \\
\text { (敞伏秒) }\end{array}$ & \% 面积 & $\begin{array}{l}\text { 高度 } \\
\text { (溦伏) }\end{array}$ & 积分类型 & 类量 & 单位 & 峰类型 & 峰代码 & $\begin{array}{l}\text { 结构 } 1 \\
\text { 名 }\end{array}$ & \begin{tabular}{|l} 
结构 1 \\
说明
\end{tabular} & $\begin{array}{l}\text { 结构 } 1 \\
\text { 分子量 }\end{array}$ & \begin{tabular}{l|l}
1 & 结构 1 \\
公式
\end{tabular} & \begin{tabular}{|l|} 
结构 1 \\
结构
\end{tabular} \\
\hline 1 & & 10.339 & 86635345 & 46.79 & 2943658 & 60 & & & 末知 & & & & & & 9 \\
\hline 2 & & 12.122 & 98527717 & 53.21 & 2789991 & $b b$ & & & 末知 & & & & & & s \\
\hline
\end{tabular}


(S)-3-(2-nitro-1-(m-tolyl)ethyl)pentane-2,4-dione (Literature: Asian J. Org. Chem. 2014, 3, 445). (HPLC: Chiracel AD-H, detected at $215 \mathrm{~nm}$, eluent: $\mathrm{n}$-hexane/2-propanol = 90/10, flow rate $=1.0 \mathrm{~mL} / \mathrm{min}, 25^{\circ} \mathrm{C}, \mathrm{t}_{1}=7.2 \mathrm{~min}(\mathrm{maj})$, $\left.\mathrm{t}_{2}=8.2 \mathrm{~min}\right)$.
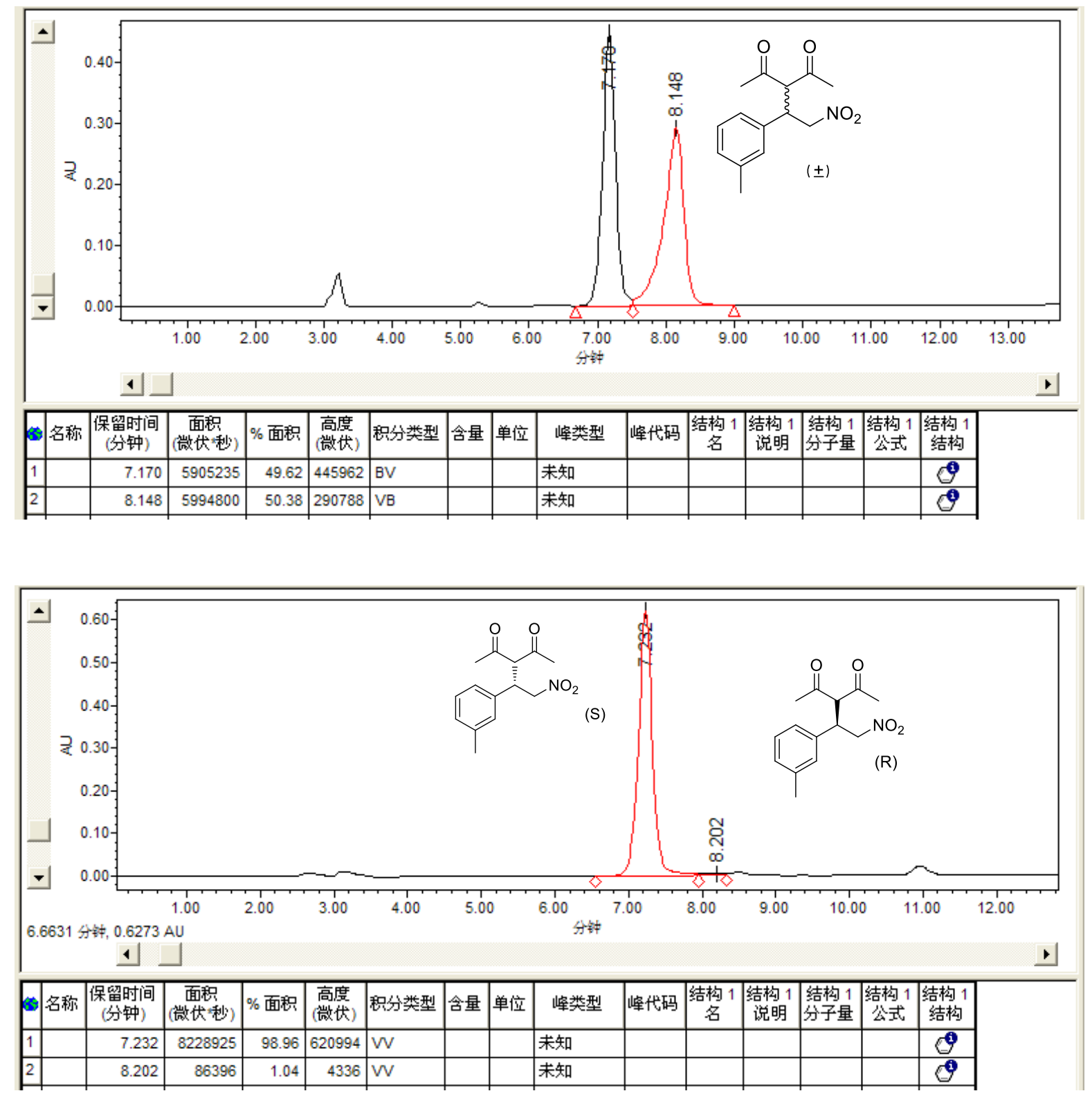

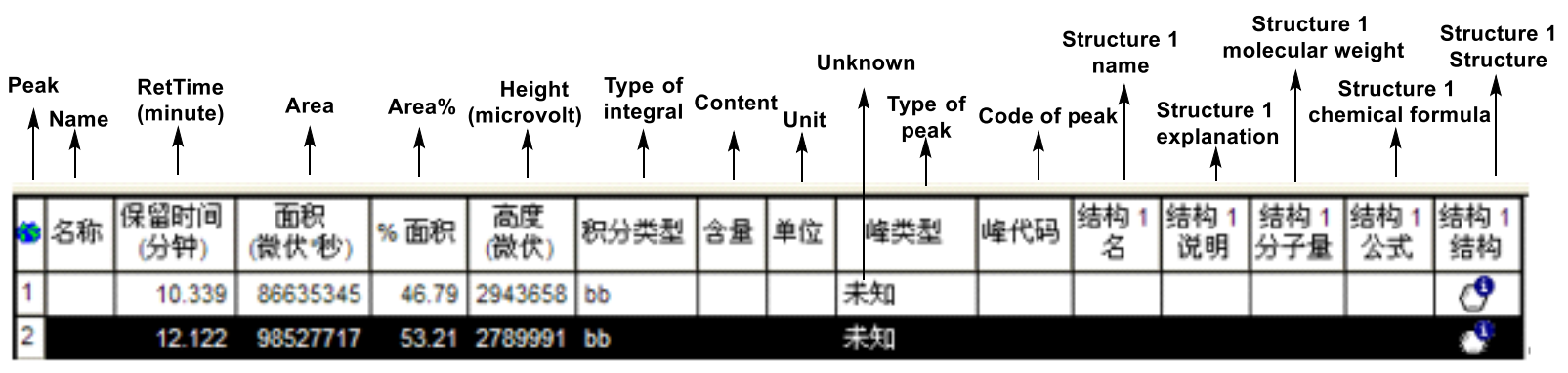


(S)-3-(1-(4-methoxyphenyl)-2-nitroethyl)pentane-2,4-dione) (Literature: ACS Catal. 2014, 4,2137). (HPLC: Chiracel AD-H, detected at $215 \mathrm{~nm}$, eluent: $n$-hexane/2-propanol $=90 / 10$, flow rate $=1.0 \mathrm{~mL} / \mathrm{min}, 25^{\circ} \mathrm{C}, \mathrm{t}_{1}=12.3 \mathrm{~min}(\mathrm{maj}), \mathrm{t}_{2}=18.0 \mathrm{~min}$.
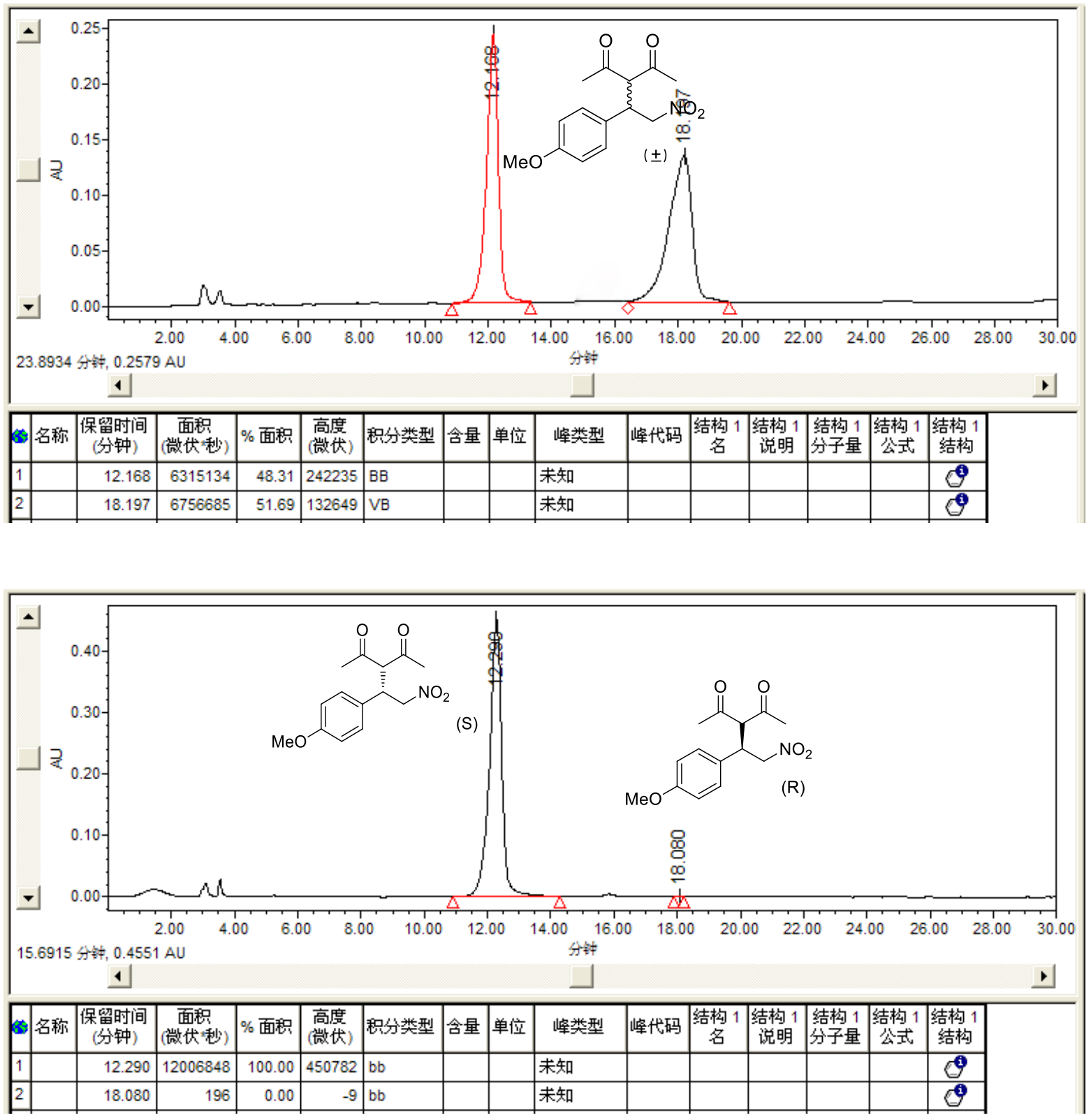

\begin{tabular}{|c|c|c|c|c|c|c|c|c|c|c|c|c|c|c|}
\hline \multirow[b]{2}{*}{$\begin{array}{l}\text { Peak } \\
\text { Na }\end{array}$} & \multirow[b]{2}{*}{$\begin{array}{c}\text { RetTime } \\
\text { (minute) }\end{array}$} & \multirow[b]{2}{*}{$\stackrel{\text { Area }}{\uparrow}$} & \multirow[b]{2}{*}{$\stackrel{\text { Area } \%}{\uparrow}$} & \multirow[b]{2}{*}{$\begin{array}{c}\text { Height } \\
\text { (microvolt) }\end{array}$} & \multirow[b]{2}{*}{$\begin{array}{c}\text { Type of } \\
\text { integral }\end{array}$} & \multicolumn{3}{|c|}{ Unknown } & \multicolumn{2}{|c|}{$\begin{array}{c}\text { Structure } 1 \\
\text { name }\end{array}$} & \multicolumn{3}{|c|}{$\begin{array}{l}\text { Structure } 1 \\
\text { molecular weight }\end{array}$} & $\begin{array}{l}\text { Structure } \\
\text { Structure }\end{array}$ \\
\hline & & & & & & $\stackrel{\uparrow}{\text { Conten }}$ & Unit & $\begin{array}{c}\text { Type of } \\
\text { peak }\end{array}$ & Code of $p$ & peak & $\begin{array}{l}\text { Structure } \\
\text { explanatic } \\
\uparrow\end{array}$ & $\begin{array}{l}\text { e } 1 \\
\text { tion }\end{array}$ & $\begin{array}{c}\text { Structure } \\
\text { hemical for }\end{array}$ & ormula \\
\hline 5 名称 & $\begin{array}{c}\text { 保留时间 } \\
\text { (分钟) }\end{array}$ & $\begin{array}{l}\text { 面知 } \\
\text { (敞伙秒) }\end{array}$ & \% 面积 & $\begin{array}{l}\text { 高度 } \\
\text { (微状) }\end{array}$ & 积分类型 & 숭量 & 单位 & 峰类型 & 峰代码 & $\begin{array}{c}\text { 结构 } 1 \\
\text { 名 }\end{array}$ & \begin{tabular}{|c|} 
结构 1 \\
说明
\end{tabular} & $\begin{array}{l}\text { 结构 } 1 \\
\text { 分子量 }\end{array}$ & $\begin{array}{c}1 \\
\text { 结构 } 1 \\
\text { 公式 }\end{array}$ & \begin{tabular}{|l} 
结构 1 \\
结构
\end{tabular} \\
\hline 1 & 10.339 & 86635345 & 46.79 & 2943658 & bo & & & 末末知 & & & & & & 9 \\
\hline 2 & 12.122 & 98527717 & 53.21 & 2789991 & bb & & & 末末知 & & & & & & 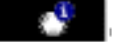 \\
\hline
\end{tabular}


(S)-3-(1-(3-methoxyphenyl)-2-nitroethyl)pentane-2,4-dione (Literature: Org. Biomol. Chem. 2015, 13, 5054). (HPLC: Chiracel AD-H, detected at $215 \mathrm{~nm}$, eluent: $\mathrm{n}$-hexane/2propanol $=99 / 1$, flow rate $\left.=1.0 \mathrm{~mL} / \mathrm{min}, 25^{\circ} \mathrm{C}, \mathrm{t}_{1}=15.8 \mathrm{~min}(\mathrm{maj}), \mathrm{t}_{2}=20.5 \mathrm{~min}\right)$.
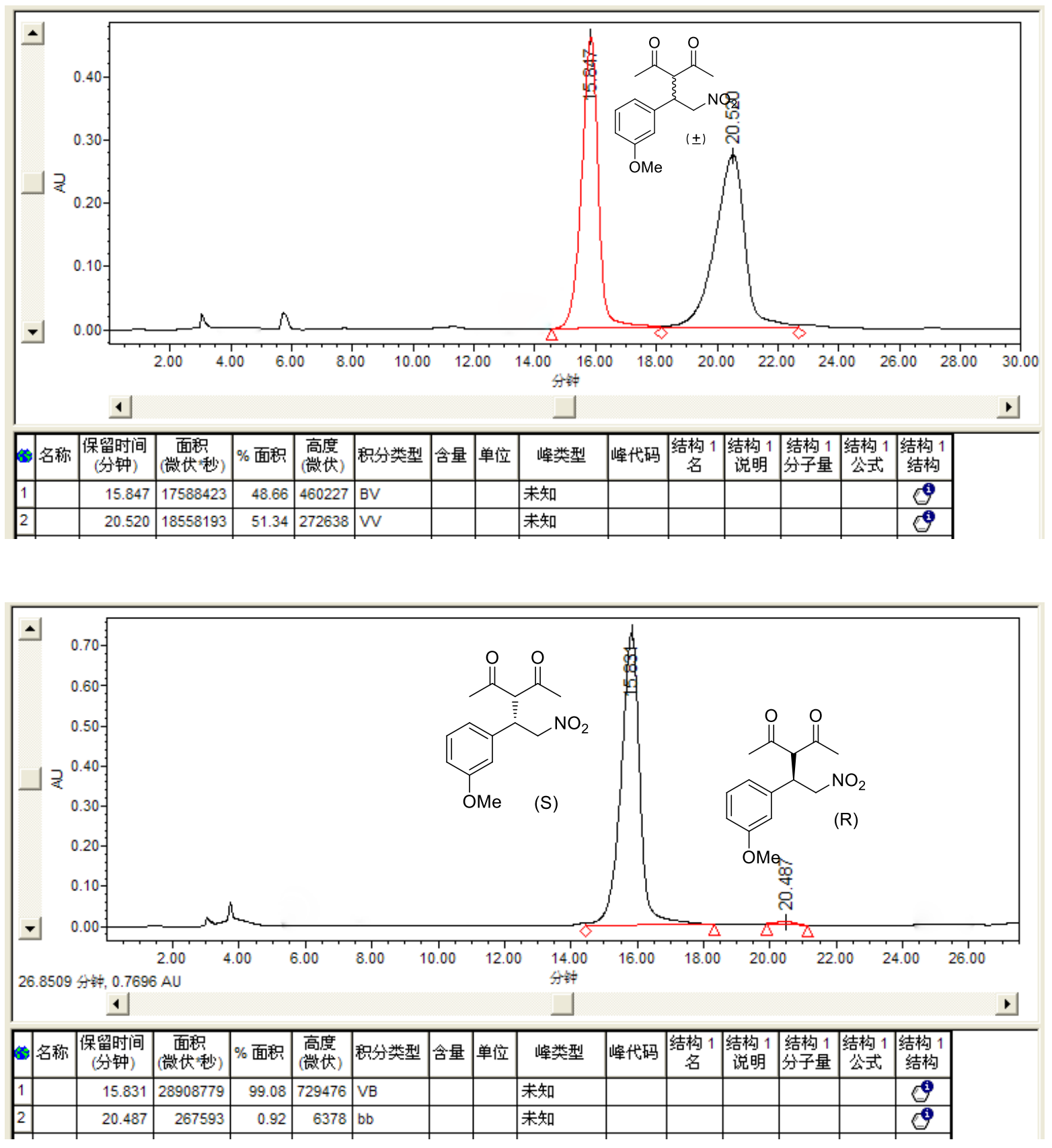

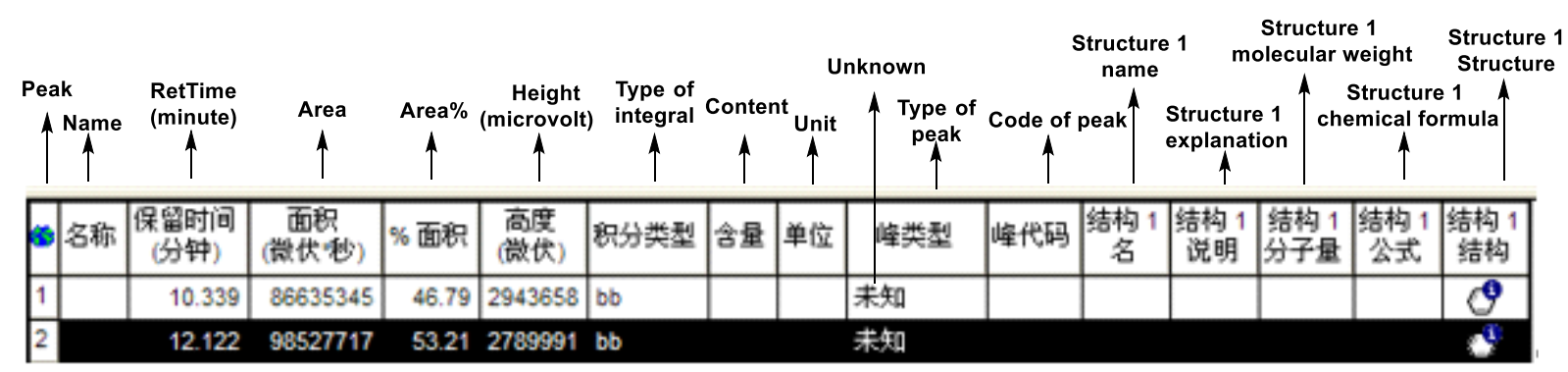


(S)-3-(1-(2-methoxyphenyl)-2-nitroethyl)pentane-2,4-dione (Literature: Green Chem. 2012, 14, 893). (HPLC: Chiracel AD-H, detected at $215 \mathrm{~nm}$, eluent: $\mathrm{n}$-hexane/2-propanol = $99 / 01$, flow rate $=1.0 \mathrm{~mL} / \mathrm{min}, 25 \stackrel{\circ}{\circ}, \mathrm{t}_{1}=11.6 \mathrm{~min}(\mathrm{maj})$, $\left.\mathrm{t}_{2}=12.0 \mathrm{~min}\right)$
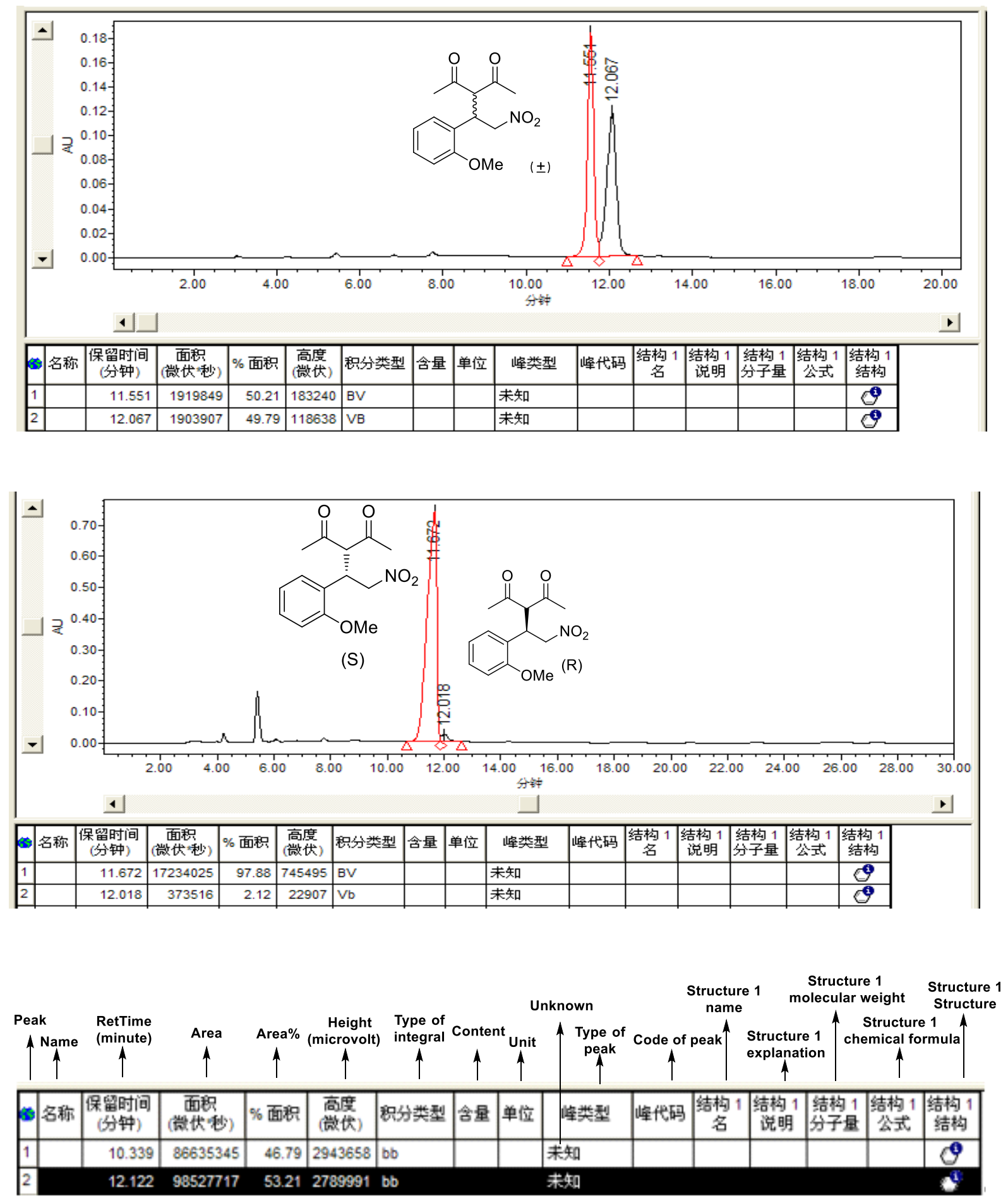

Figure S10. Asymmetric Michael addition of acetylacetone to nitroalkenes. [The products were analyzed by a HPLC with a UV-Vis detector using a Daicel AD-H, OD-H, AS-H or OJ$\mathrm{H}$ chiralcel column $(\Phi 0.46 \times 25 \mathrm{~cm})$. 
Table S2. Reusability of catalyst $\mathbf{5}$ for asymmetric Michael addition of acetylacetone to nitrostyrene. (Reaction conditions: catalyst 5 (362.6. $\mathrm{mg}, 50.0 \mu \mathrm{mol}$ of squaramide based on TG analysis), nitroalkenes (10.0 mmol), acetylacetone $(20.0 \mathrm{mmol}), 20.0 \mathrm{~mL}$ brine, reaction time (20 minute). The ee values were determined by chiral HPLC analysis.)

\begin{tabular}{lcccccccc}
\hline Entry & $\mathbf{1}$ & $\mathbf{2}$ & $\mathbf{3}$ & $\mathbf{4}$ & $\mathbf{5}$ & $\mathbf{6}$ & $\mathbf{7}$ & $\mathbf{8}$ \\
\hline Conversion [\%] & 99 & 99 & 99 & 97 & 99 & 98 & 96 & 92 \\
ee [\%] & 99 & 99 & 99 & 97 & 97 & 97 & 97 & 97 \\
\hline
\end{tabular}

Recycling experiment part: Recycle 1

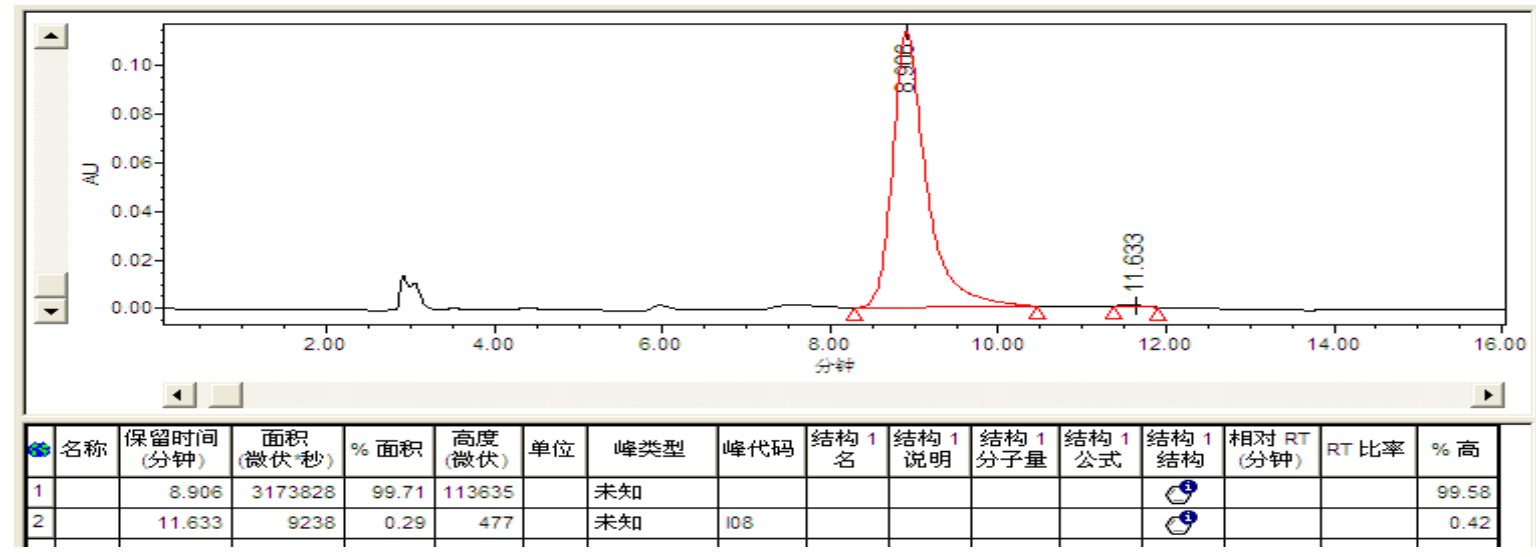

Recycle 2

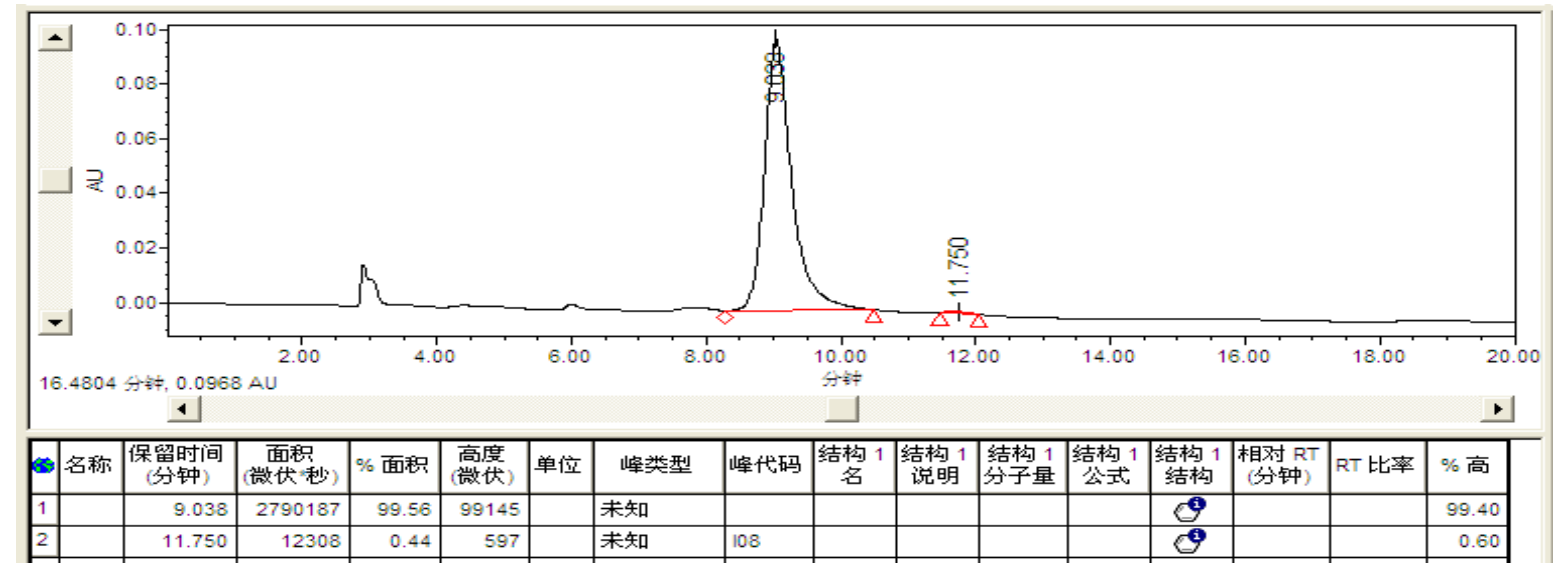

\begin{tabular}{|c|c|c|c|c|c|c|c|c|c|c|c|c|c|c|c|c|}
\hline \multirow{2}{*}{\multicolumn{2}{|c|}{$\uparrow_{\uparrow}^{\text {Peak }} \uparrow^{\text {Name }}$}} & \multirow{2}{*}{$\begin{array}{l}\text { RetTime } \\
\text { (minute) } \\
\uparrow \text { (micr }\end{array}$} & \multirow{2}{*}{\multicolumn{2}{|c|}{ 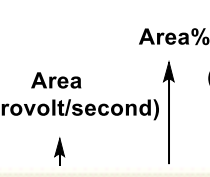 }} & \multirow{2}{*}{\multicolumn{3}{|c|}{$\begin{array}{l}\text { eight } \\
\text { tovolt) }\end{array}$}} & \multicolumn{5}{|c|}{$\begin{array}{cc}\text { Structure } 1 & \text { Structure } 1 \\
\text { name } & \text { molecular weight }\end{array}$} & \multicolumn{4}{|c|}{$\begin{array}{l}\text { Structure } 1 \\
\text { Structure } \\
\text { Relative }\end{array}$} \\
\hline & & & & & & & & $\begin{array}{c}\text { Code of } \\
\text { peak }\end{array}$ & $\left\lceil\begin{array}{l}\text { Str } \\
\text { ex }\end{array}\right.$ & $\begin{array}{l}\text { tructure } 1 \\
\text { xplanation }\end{array}$ & n $\uparrow$ che & $\begin{array}{l}\text { Structure } \\
\text { mical for }\end{array}$ & ormula & $\begin{array}{l}\text { RetTime } \\
\text { (minute) }\end{array}$ & $\begin{array}{l}\text { Ratio of } \\
\text { RetTime }\end{array}$ & f \\
\hline 3 & 名称 & 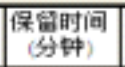 & 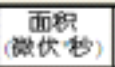 & $\%$ 面的 & $\begin{array}{l}\text { 裔度 } \\
\text { (做仗) }\end{array}$ & 事位 & 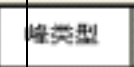 & 俚代的 & \begin{tabular}{|l|} 
结构 1 \\
名
\end{tabular} & 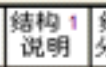 & $\begin{array}{l}\text { 结禡 } 1 \\
\text { 分子量 }\end{array}$ & \begin{tabular}{|c|} 
结构 1 \\
公式
\end{tabular} & \begin{tabular}{|l|} 
結私 1 \\
结构
\end{tabular} & 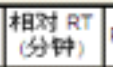 & RT 比雪 & \%高 \\
\hline 1 & & 8.906 & 3173828 & 99.71 & 113635 & & 来知 & & & & & & 6 & & & 99.58 \\
\hline 2 & & 11.633 & 9238 & 0.29 & 477 & & 来知 & 108 & & & & & 6 & & & 0.42 \\
\hline
\end{tabular}


Recycle 3

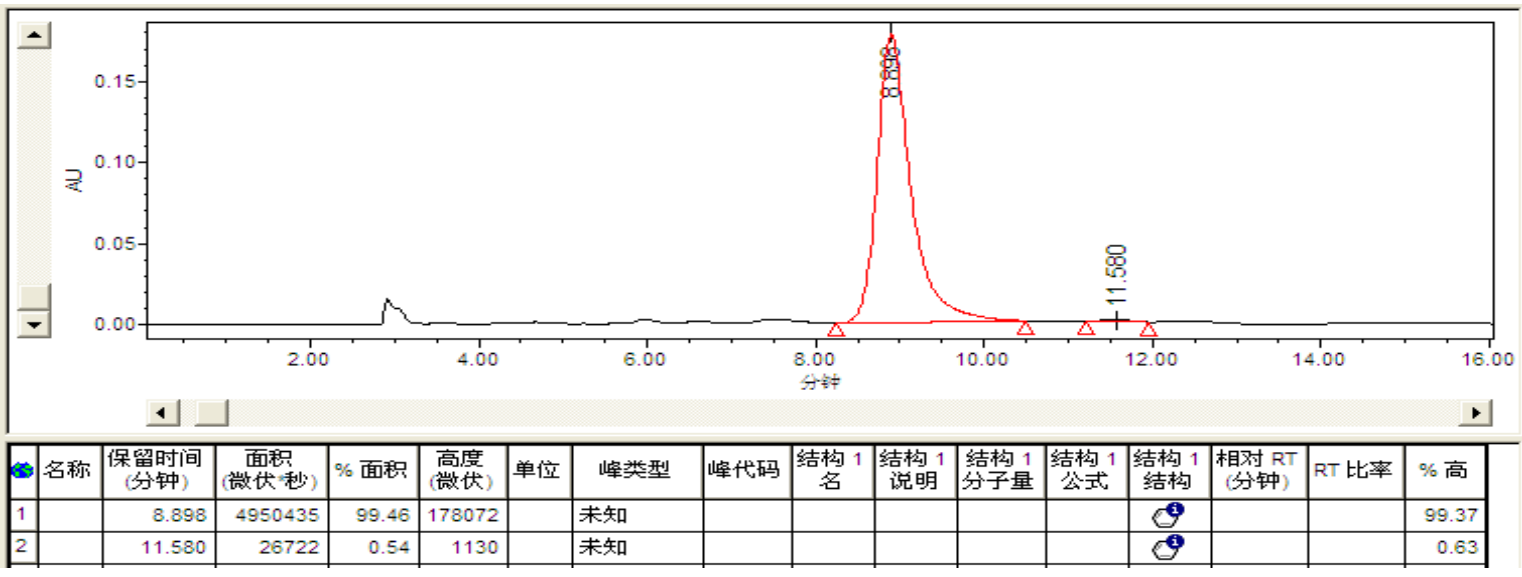

\section{Recycle 4}

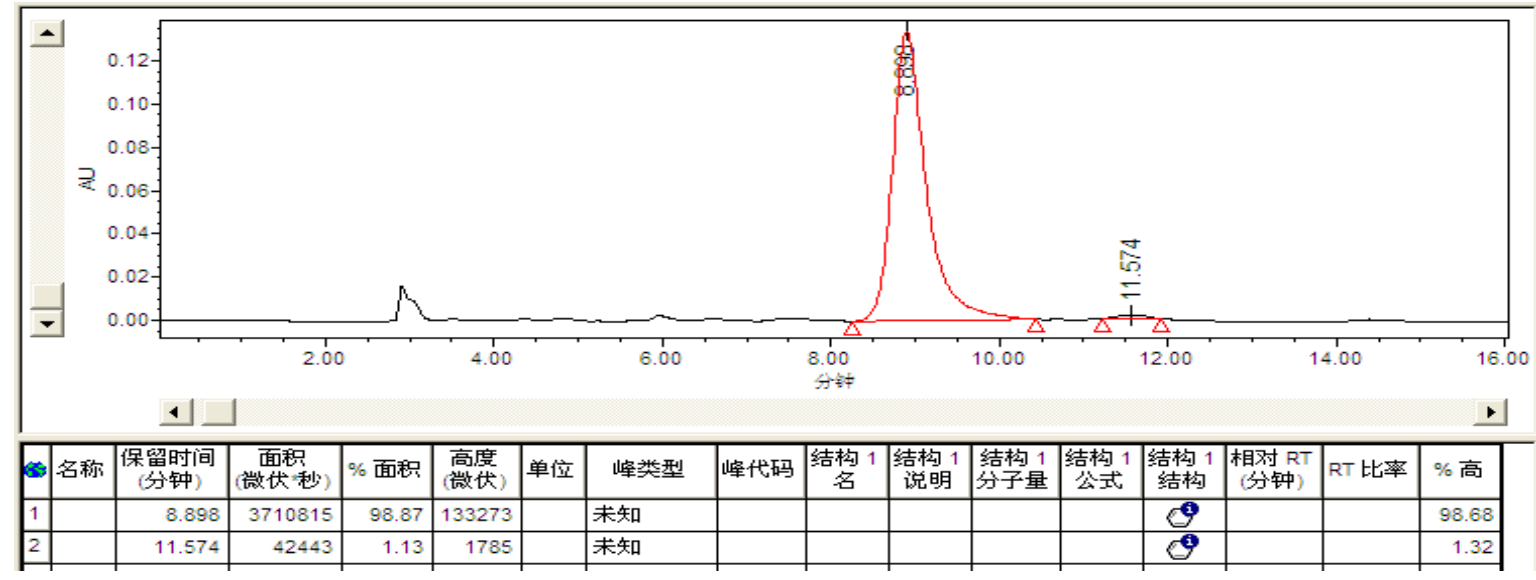

\section{Recycle 5}

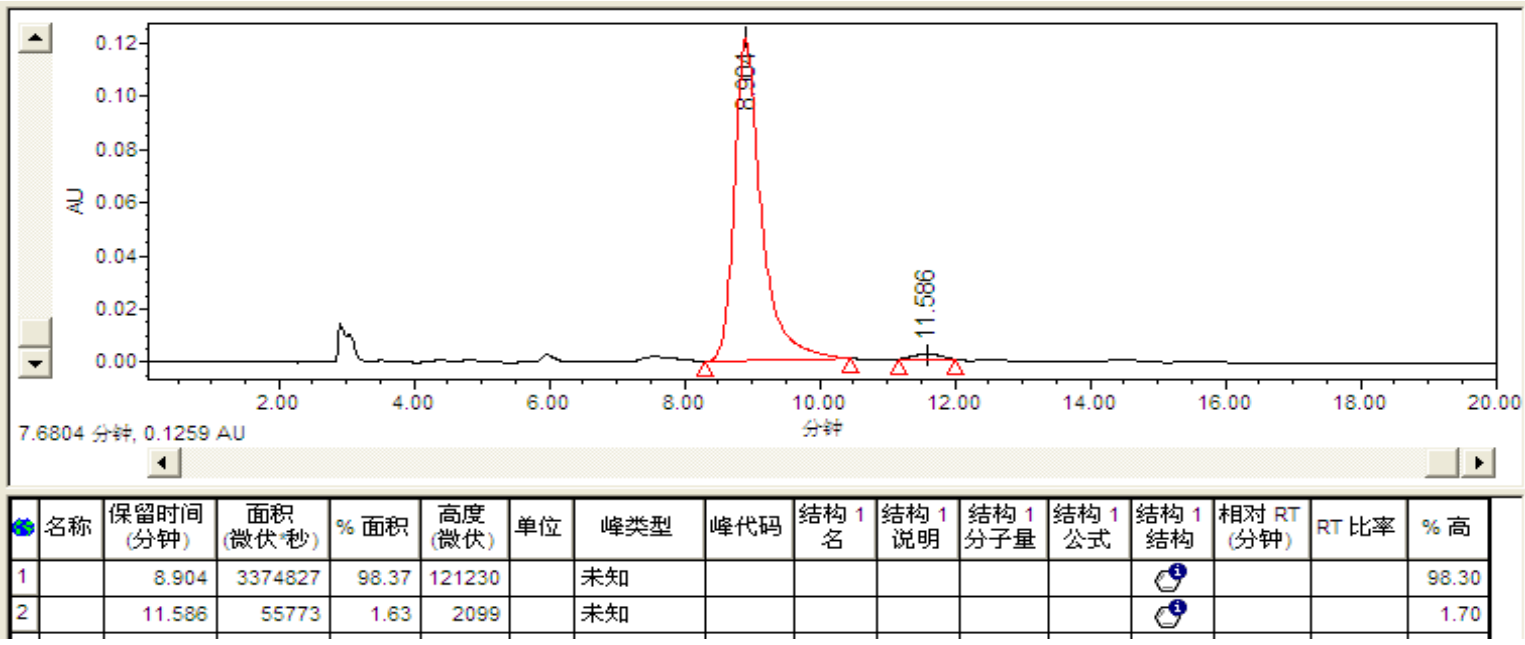

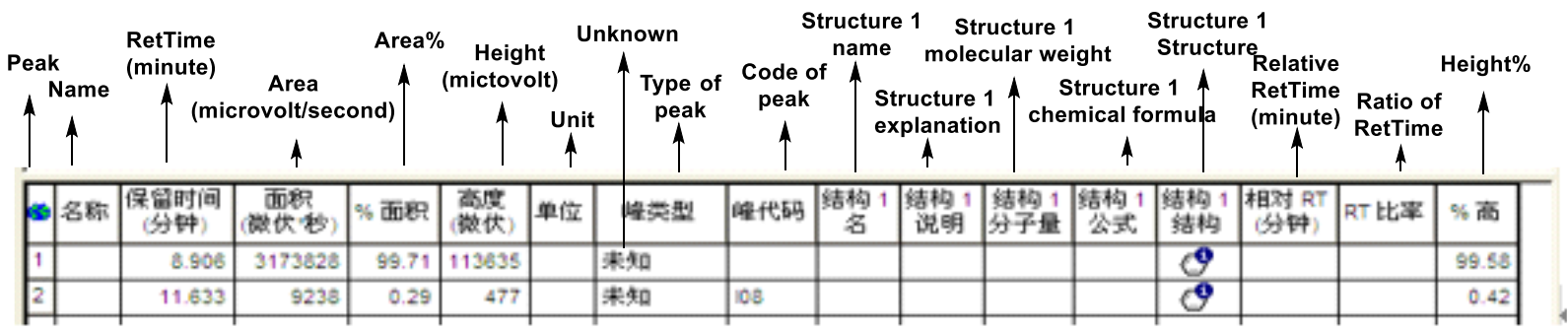


Recycle 6

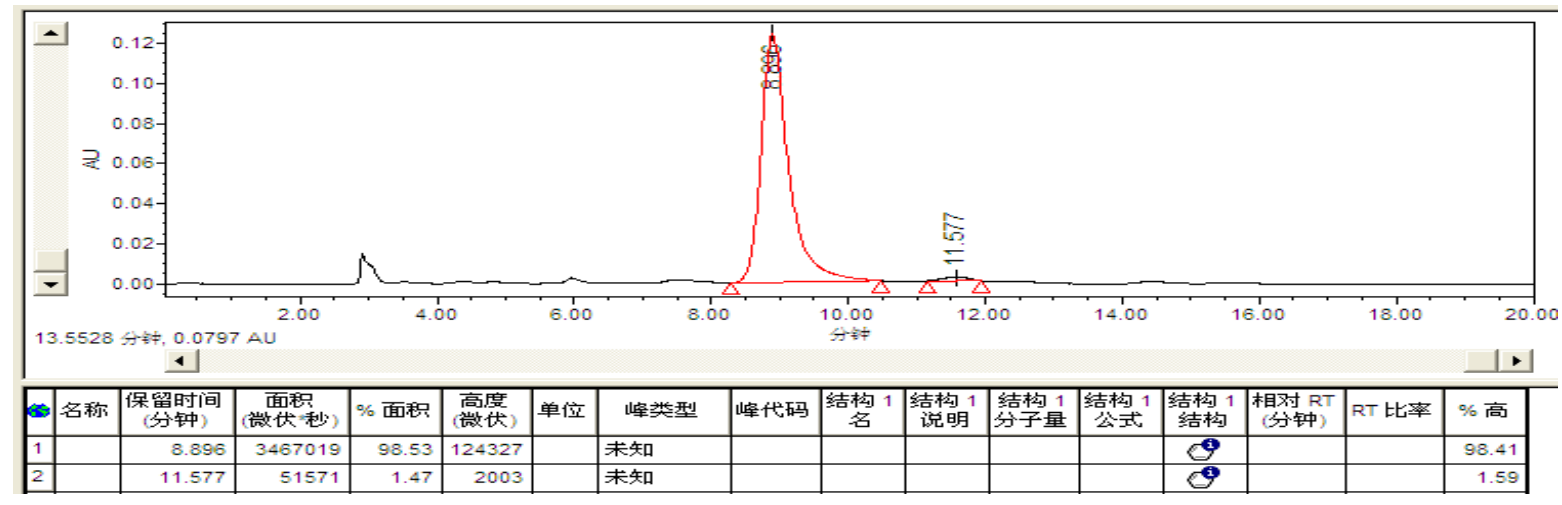

Recycle 7

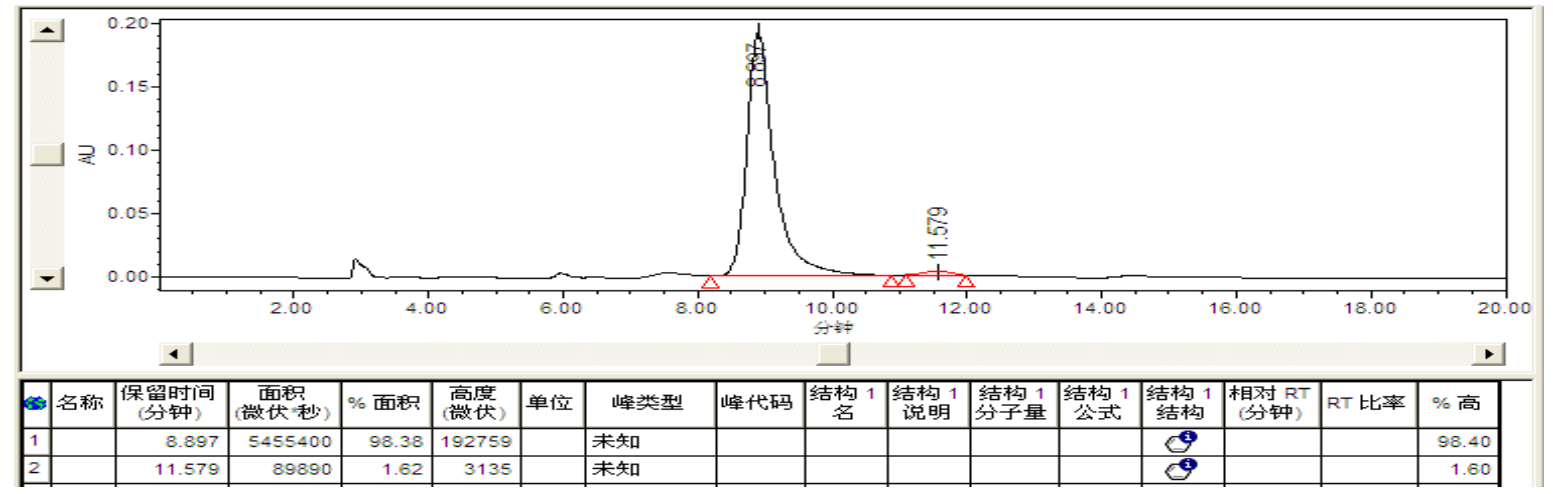

Recycle 8

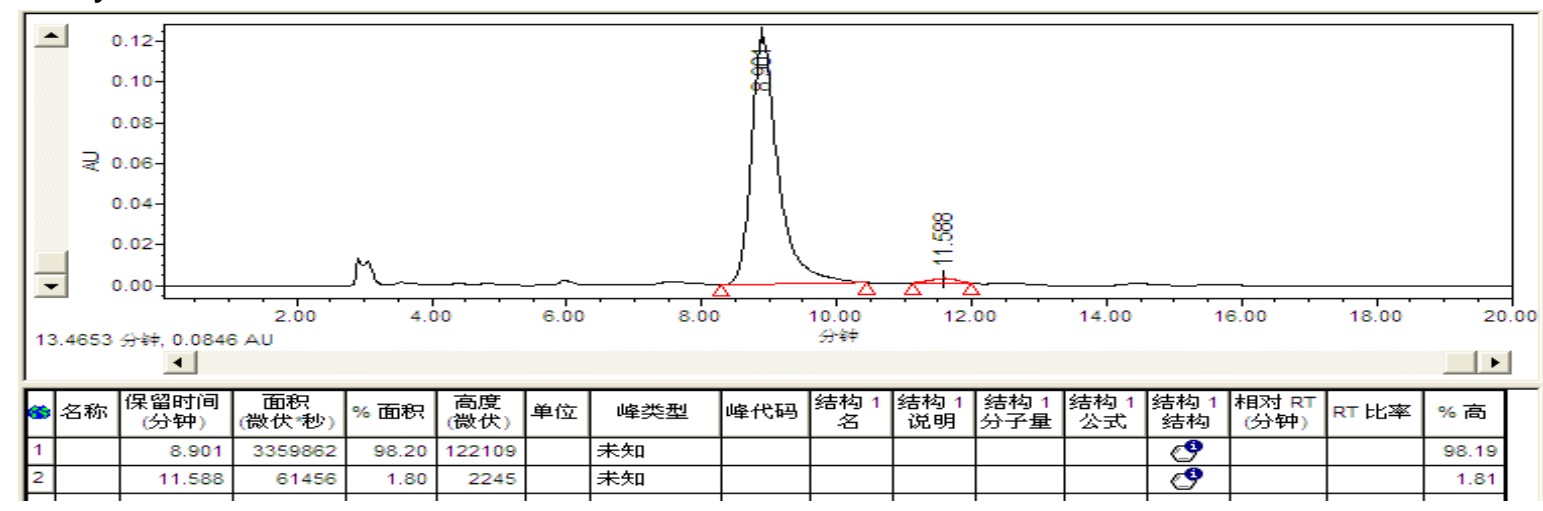

RetTime
(minute)

Figure S11. Reusability of catalyst $\mathbf{5}$ for asymmetric Michael addition of acetylacetone to nitrostyrene. 